Métodos numéricos para escoamentos com linhas de contato dinâmicas

Felipe Montefuscolo 

Data de Depósito:

Assinatura:

\title{
Métodos numéricos para escoamentos com linhas de contato dinâmicas
}

\author{
Felipe Montefuscolo
}

Orientador: Prof. Dr. Fabrício Simeoni de Sousa

Dissertação apresentada ao Instituto de Ciências Matemáticas e de Computação - ICMC-USP, como parte dos requisitos para obtenção do título de Mestre em Ciências - Ciências de Computação e Matemática Computacional. VERSÃO REVISADA

USP - São Carlos

Maio de 2012 
Ficha catalográfica elaborada pela Biblioteca Prof. Achille Bassi e Seção Técnica de Informática, ICMC/USP, com os dados fornecidos pelo(a) autor(a)

\begin{tabular}{|c|c|}
\hline \multirow[t]{3}{*}{ M774m } & $\begin{array}{l}\text { Montefuscolo, Felipe } \\
\text { Métodos numéricos para escoamentos com linhas de } \\
\text { contato dinâmicas / Felipe Montefuscolo; orientador } \\
\text { Fabrício Simeoni de Sousa. -- São Carlos, } 2012 \text {. } \\
\quad 93 \text { p. }\end{array}$ \\
\hline & $\begin{array}{l}\text { Dissertação (Mestrado - Programa de Pós-Graduação en } \\
\text { Ciências de Computação e Matemática Computacional) -- } \\
\text { Instituto de Ciências Matemáticas e de Computação, } \\
\text { Universidade de São Paulo, } 2012 \text {. }\end{array}$ \\
\hline & $\begin{array}{l}\text { 1. Método dos elementos finitos. 2. Lagrangeano- } \\
\text { euleriano arbitrário. 3. Tensão superficial. } 4 \text {. } \\
\text { Linha de contato. 5. Capilaridade. I. Simeoni de } \\
\text { Sousa, Fabrício, orient. II. Título. }\end{array}$ \\
\hline
\end{tabular}


Que bom que nós nos encontramos com um paradoxo.

Agora temos alguma esperança de progredir.

Niels Bohr. 



\section{Resumo}

O fenômeno de molhamento, estudo de como um líquido se deposita em um sólido, apresenta problemas ainda em aberto, dos pontos de vista da modelagem física e da simulação numérica. O maior interesse acadêmico neste tipo de escoamento é a linha tríplice (ou linha de contato) formada da interação sólido-líquido-gás. A condição de contorno clássica de não escorregamento na interface líquido-sólido leva a uma singularidade no tensor de tensões nesta linha. Além disso, ainda não está estabelecido qual o melhor modelo para descrever o ângulo de contato formado entre a superfície livre e o substrato (o sólido).

Neste trabalho, são discutidos métodos numéricos para a simulação de linhas de contato dinâmicas. Os efeitos da tensão superficial são estudados com a abordagem do princípio do trabalho virtual, o qual leva o problema à equações na formulação variacional, linguagem natural para o tratamento numérico com o método dos elementos finitos (FEM). O domínio é discretizado por uma malha não-estruturada de forma que as interfaces separadoras são explicitamente representadas pela malha. As derivadas temporais são tratadas em uma abordagem Lagrangeana-Euleriana arbitrária (ALE).

Finalmente, são apresentados os resultados numéricos obtidos com o método ALE-FEM, discutindo alguns aspectos da sua convergência temporal e espacial. 



\section{Abstract}

Wetting phenomena, study of how of a liquid spreads out on a solid substrate, presents challenges both in physical modeling and in numerical simulation. The triple line (or contact line) formed by the solid-liquid-gas interaction has increasingly attracted the attention of the fluid dynamic community. The classical no-slip boundary condition on the liquid-solid interface leads to a singularity in the stress tensor at contact lines. Furthermore, there is no consensus on what the best model to describe the dynamics of the contact angle formed by the solid substrate and free surface.

In this work, numerical methods for simulating dynamic contact lines are considered. The capillarity effects are studied in the approach of the virtual-work principle, which describes the problem in the variational formulation, natural language for numerical treatment with the finite element method (FEM). The domain is discretized by a dynamic unstructured mesh, where the separating interfaces are explicit represented by the mesh. Time derivatives present in the governing equations are treated with the arbitrary Lagrangian-Eulerian (ALE) framework.

Finally, we discuss some temporal and spatial convergence issues of the ALE-FEM method. 



\section{Sumário}

Lista de Figuras

Lista de Tabelas

1 Introdução $\quad 17$

1.1 Visão geral . . . . . . . . . . . . . . . . . . . . . . . . . . 17

1.2 Objetivos da dissertação . . . . . . . . . . . . . . . . . . . . . . 18

2 Método dos elementos finitos na formulação ALE $\quad 19$

2.1 Descrição cinemática na formulação ALE . . . . . . . . . . . . . . . . . 19

2.2 Formulação variacional das equações de Navier-Stokes . . . . . . . . . . 22

2.2.1 Problema contínuo . . . . . . . . . . . . . . 22

2.2.2 Discretização espacial . . . . . . . . . . . . . . . . . 24

2.2 .3 Discretização temporal . . . . . . . . . . . . . . . . 26

2.2 .4 Sistema de equações . . . . . . . . . . . . . . . . . . 32

2.3 Elementos adotados . . . . . . . . . . . . . . . . . . . . 32

2.4 A condição de contorno de não penetração . . . . . . . . . . . . . . . . 38

2.4.1 Rotações/Projeções . . . . . . . . . . . . . . . . . . . . . 38

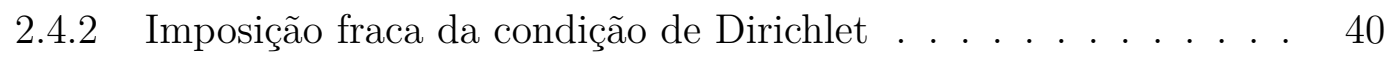

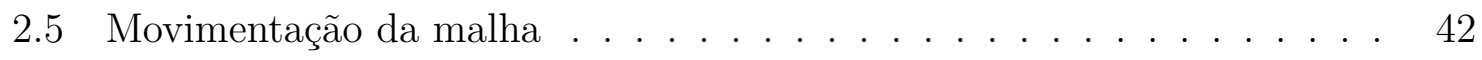

2.5.1 Suavização Laplaciana restrita . . . . . . . . . . . . . . . . . . 42

2.5.2 Medida de qualidade . . . . . . . . . . . . . . . 43

2.5.3 Suavização no bordo e normais . . . . . . . . . . . . . . . . 44

2.5.4 Observações . . . . . . . . . . . . . . . . . . . . . 46 
3 Exemplos e testes numéricos para a formulação ALE 49

3.1 Validações . . . . . . . . . . . . . . . . . . . . . . . . 50

3.1 .1 Convergência espacial em 2D . . . . . . . . . . . 50

3.1.2 Convergência temporal . . . . . . . . . . . . . . . . . . . 52

4 Elementos de geometria diferencial $\quad 59$

4.1 Extensão e função distância . . . . . . . . . . . . . . . . . . . . . . . . 59

4.2 Operadores diferencias na superfície . . . . . . . . . . . . . . 60

4.3 Curvaturas e formas fundamentais . . . . . . . . . . . . . . . . 61

4.4 Outros fatos e definições . . . . . . . . . . . . . . . . . . . . 62

4.5 Jacobiana de transformações em integrais . . . . . . . . . . . . . . . 63

5 Forças capilares, molhamento e ângulo de contato 65

5.1 Introdução . . . . . . . . . . . . . . . . . . . . . . . . . 65

5.2 O princípio das potências virtuais . . . . . . . . . . 66

5.3 Potências virtuais na superfície . . . . . . . . . . . . . . 67

5.4 Potências virtuais na linha de contato . . . . . . . . . . . . . . 70

5.5 Singularidade, forças dissipativas e ângulo de contato dinâmico . . . . . 70

5.6 Formulação variacional . . . . . . . . . . . . . . . . . . . 72

5.7 Dificuldades e outros modelos . . . . . . . . . . . . . . . . . . . 72

6 Aspectos computacionais e exemplos numéricos $\quad 75$

6.1 Discretização e métodos . . . . . . . . . . . . . . . . 75

6.1.1 Formulação variacional discreta final . . . . . . . . . . . . . 75

6.1.2 Cálculos nas superfícies e linha de contato. . . . . . . . . . . 76

6.2 Exemplos numéricos . . . . . . . . . . . . . . . . . . . . 77

6.2.1 Superfície livre . . . . . . . . . . . . . . . 77

6.2.2 Gota estática . . . . . . . . . . . . . . . . 78

6.2.3 Gota oscilante . . . . . . . . . . . . . . . . . . 81

6.2.4 Ângulo estático . . . . . . . . . . . . . . . . . . . 82

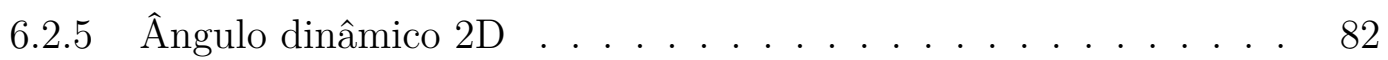

6.2 .6 Outros testes . . . . . . . . . . . . . . . . . 83

$\begin{array}{lll}7 & \text { Conclusão } & 87\end{array}$

$\begin{array}{lr}\text { Referências Bibliográficas } & 89\end{array}$ 


\section{Lista de Figuras}

2.1 Esboço do mapeamento entre os domínios espacial, material e referencial.

2.2 Exemplo de reposicionamento do vértice via suavização Laplaciana: caso convexo e não convexo (figura retirada de Sousa [46]). O vetor $\hat{\mathbf{x}}^{i}$ é a nova posição do vértice. . . . . . . . . . . . . . . . . . . . . . 4 43

2.3 Orientação dos vértices adotada para as fórmulas de qualidade. . . . . . . 44

2.4 Ilustração das variáveis necessárias para se calcular a normal $\mathbf{n}^{i}$ no vértice $i$, usando a fórmula proposta em $\operatorname{Max}[35] \ldots$. . . . . . . . . . . . . 46

2.5 Nós característicos (realçados em vermelho) . . . . . . . . . . . . . 48

3.1 Erros da velocidade e pressão no problema de Kovasznay. . . . . . . . . . . 51

3.2 Movimento da malha nos testes de convergência temporal referente a velocidade em (3.6). Na primeira linha, a malha é movimentada sob deformações lineares ao passo que na segunda, a malha é movimentada sob deformações quadráticas. . . . . . . . . . . . . . . . . 53

3.3 Comparação dos erros da pressão em $L^{2}$ (esquerda) e $H^{1}$ (direita) para o método de Crank-Nicolson em uma malha fixa entre a pressão computada no passo de tempo $n+1$ e a computada em $n+1 / 2 \ldots \ldots \ldots 4$

3.4 Testes de convergência temporal dos problemas (A) e (B) na formulação ALE. As abreviações "lin" e "quad" significam elementos lineares e curvos, respectivamente. . . . . . . . . . . . . . . . . .

3.5 Movimentação da malha no teste de convergência das posições nodais. O nó ressaltado nas figuras é o nó acompanhado durante a simulação. . . . . 56

3.6 Testes de convergência das posições nodais da malha no problema (C). . . 57 
4.1 Ilustração de uma superfície $\Gamma$, da região $\mathcal{D}$ que a envolve, de sua fronteira $\partial \Gamma$, da normal $\mathbf{n}$, da conormal $\boldsymbol{\nu}$ e da projeção normal $\Pi_{\Gamma}$ de um ponto $\boldsymbol{x}$.

5.1 Na esquerda, está um caso de uma gota livre, enquanto que na direita, está um caso de uma gota em contato com um substrato sólido (a parte hachurada). Os pontos em vermelho representam a linha de contato que é denotada por $\partial \Gamma \ldots \ldots \ldots \ldots \ldots \ldots \ldots \ldots$

5.2 Ilustração da conormal $\boldsymbol{\nu}$ da superfície $\Gamma$, que é perpendicular à normal de $\Gamma$ e normal à linha de contato, e da conormal $\boldsymbol{\nu}_{s}$ da superfície sólida $\Gamma_{s}$, que é perpendicular à normal de $\Gamma_{s}$ de normal à linha de contato. O ângulo $\theta$ é igual ao ângulo entre $\boldsymbol{\nu}_{\mathrm{e}} \boldsymbol{\nu}_{s} \ldots \ldots \ldots$. . . . . . . . . . . . . . 70

6.1 Conormal de $\Gamma_{s}$, denotada por $\boldsymbol{\nu}_{s}$, em 3D (à esquerda) e 2D (à direita). . . 76

6.2 Sloshing: campos de pressão e velocidade em vários instantes. A pressão vermelha indica a pressão mais alta. . . . . . . . . . . . . . . 78

6.3 Erros em $L^{2}$ da velocidade e pressão para vários elementos em 2D. . . . . . 79

6.4 Erros em $L^{2}$ da velocidade e pressão para vários elementos em 3D. . . . . . 80

6.5 Oscilação de uma gota inicialmente perturbada elipticamente. As cores indicam a pressão. . . . . . . . . . . . . . . . . . . . . . 81

6.6 Comparação da amplitude da gota com a solução exata. . . . . . . . . . . . 81

6.7 Validação do ângulo estático comparando a solução obtida com a solução de uma EDO. . . . . . . . . . . . . . . . . . . . . . . . 82

6.8 Ângulo de contato dinâmico com dissipação $\beta=10^{-5}$ na superfície sólidolíquido, para dois valores diferentes de dissipação na linha de contato. . . .

6.9 Dinâmica do ângulo de contato, em vários instantes diferentes. A cores indicam os valores da pressão. . . . . . . . . . . . . . . . . . . . . 83

6.10 Ângulo de contato dinâmico de uma gota 3D, com ângulo estático de $135^{\circ} . \quad 84$

6.11 Gota pendente bidimensional em vários passos de tempo, com ângulo estático de $90^{\circ}$. As cores indicam a pressão. . . . . . . . . . . . . . . . .

6.12 Gota pendente tridimensional em vários passos de tempo, com ângulo estático de $45^{\circ}$. As cores indicam a magnitude da velocidade que está alinhada

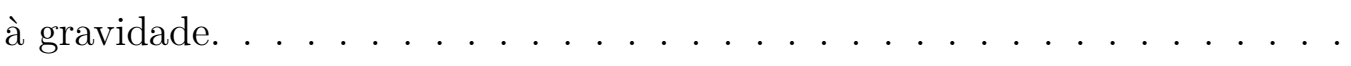




\section{Lista de Algoritmos}

2.1 Solução $\mathbf{u}_{h}, p_{h}$ e $\mathbf{x}_{h}$ a cada passo de tempo, via ALE. . . . . . . . . . . 31

2.2 Suavização da malha. . . . . . . . . . . . . . . . . . 47

\section{Lista de Tabelas}

3.1 Siglas dos elementos. . . . . . . . . . . . . . . . . . . 50 



\section{Capítulo 1}

\section{Introdução}

\section{$1.1 \quad$ Visão geral}

A compreensão dos fenômenos tensão superficial, capilaridade e molhamento são pertinentes a inúmeras áreas industriais como na fabricação de veículos, indústria de alimentos, vidros e, mais recentemente, na fabricação dos Lab-On-a-Chip, microdispositivos que englobam, entre outros, os dispositivos microfluídicos.

Molhamento é o efeito de como um líquido se deposita em um sólido. Esse fenômeno, ainda que passe despercebido, é visto no cotidiano das pessoas como a trajetória que uma simples gota de água faz em um vidro sob a ação da gravidade. Linhas de pesquisa interdisciplinares como deste trabalho, onde são considerados problemas em pequenas escalas (desde $\mu m$ até $m m$ no caso), abrem inúmeras portas para descobertas científicas e são assuntos recentes. O desafio acadêmico do fenômeno de molhamento vai desde encontrar um modelo físico que prediz corretamente o comportamento do escoamento, por exemplo, uma descrição para as linhas de contato dinâmicas, até desenvolver métodos numéricos que levam um modelo complexo ao computador, por exemplo, métodos para tratar domínios não fixos e descontinuidades nas variáveis do modelo.

O centro das atenções na simulação numérica do molhamento são as linhas de contato, principal objeto de estudo deste projeto. Linha de contato é a linha formada pela interseção das regiões entre fluido-gás-sólido ou fluido-fluido-sólido. Uma das principais dificuldades em simulações de escoamentos onde estão presentes linhas de 
contato dinâmicas, é o fato de que a clássica condição de contorno das equações de Navier-Stokes, a condição de não escorregamento, leva a uma singularidade das tensões na linha de contato.

\subsection{Objetivos da dissertação}

Um método lagrangeano-euleriano arbitrário para a resolução de escoamentos dominados por tensão superficial é apresentado. São feitas revisões sobre referenciais arbitrários no contexto do método de elementos finitos, percorrendo alguns detalhes sobre os espaços discretos e discretização temporal. São também introduzidos alguns elementos de geometria diferencial para se trabalhar com superfícies e linhas de contato representadas pela malha. O objetivo principal é reunir todos estes ingredientes matemáticos e computacionais para simular escoamentos onde estão presentes as linhas de contato. 


\section{Capítulo 2}

\section{Método dos elementos finitos na formulação ALE}

\subsection{Descrição cinemática na formulação ALE}

Simulações numéricas de problemas em dinâmica dos fluidos e mecânica dos sólidos muitas vezes exigem lidar com grandes distorções do meio em consideração, mantendo uma representação precisa das fronteiras móveis e deformáveis. A idéia da formulação ALE (acrônimo na língua inglesa para Lagrangeano-Euleriano arbitrário) é introduzir um referencial arbitrário por meio de uma malha computacional que não está nem fixo no espaço (descrição Euleriana) e nem fixo no meio material (descrição Lagrangeana), de modo que a malha se acomode da melhor forma possível ao meio e à interface.

Na descrição Lagrangeana, é definido um domínio material $\Omega_{\boldsymbol{X}} \subset \mathbb{R}^{d}$, com $d$ dimensões espaciais, onde um vetor posição $\boldsymbol{X} \in \Omega_{\boldsymbol{X}}$ determina unicamente uma partícula material. Nessa descrição, as propriedades físicas do corpo são especificadas em função das coordenadas materias, $\boldsymbol{X}$, e do tempo $t \in \mathbb{R}^{+}$, e cada nó da malha computacional segue a partícula material correspondente, cuja trajetória é dada por meio de uma aplicação difeomórfica parametrizada no tempo, $\boldsymbol{\varphi}$, tal que

$$
\begin{aligned}
\boldsymbol{\varphi}(\cdot, t): \Omega_{\boldsymbol{X}} & \longrightarrow \Omega=\boldsymbol{\varphi}\left(\Omega_{\boldsymbol{X}}, t\right), \quad \forall t \geqslant 0 \\
\boldsymbol{X} & \longmapsto \boldsymbol{x}=\boldsymbol{\varphi}(\boldsymbol{X}, t), \quad \forall \boldsymbol{X} \in \Omega_{\boldsymbol{X}},
\end{aligned}
$$


onde $\boldsymbol{x}$ é a coordenada do domínio espacial $\Omega \subset \mathbb{R}^{d}$. Na descrição Euleriana, as propriedades físicas do corpo são especificadas em função das coordenadas espaciais $\boldsymbol{x}$ e do tempo $t$, e a malha computacional é fixa no espaço.

A velocidade de uma partícula especificada pela coordenada $\boldsymbol{X}=\boldsymbol{\varphi}^{-1}(\boldsymbol{x}, t)$, chamada de velocidade material, é dada pela taxa de variação temporal da posição correspondente, i.e.,

$$
\mathbf{u}^{* *}(\boldsymbol{X}, t)=\mathbf{u}(\boldsymbol{x}, t)=\left.\frac{\partial \boldsymbol{\varphi}}{\partial t}\right|_{\boldsymbol{X}},
$$

onde o asterisco é para enfatizar que a forma das funções são, em geral, diferentes. A derivada temporal de uma quantidade escalar física $\alpha^{* *}(\boldsymbol{X}, t)=\alpha(\boldsymbol{x}, t)$ em uma partícula $\boldsymbol{X}$, é dada por

$$
\left.\frac{\mathrm{D} \alpha}{\mathrm{D} t} \doteq \frac{\partial \alpha}{\partial t}\right|_{\boldsymbol{X}}=\frac{\partial \alpha}{\partial t}+\mathbf{u} \cdot \nabla \alpha
$$

e é chamada de derivada material ou total. Na descrição ALE, é introduzido um terceiro referencial $\Omega_{\chi} \subset \mathbb{R}^{d}$, que não é fixo no espaço nem no material, tal que a coordenada de referência $\chi \in \Omega_{\chi}$ identifica unicamente cada ponto da malha. Analogamente ao mapeamento Lagrangeano-Euleriano em (2.1), é possível definir um mapeamento difeomórfico $\hat{\varphi}$ entre o domínio referencial e o domínio espacial, parametrizado no tempo $t$, tal que

$$
\begin{aligned}
\hat{\boldsymbol{\varphi}}(\cdot, t): \Omega_{\chi} \longrightarrow \Omega & =\hat{\boldsymbol{\varphi}}\left(\Omega_{\chi}, t\right), \quad \forall t \geqslant 0 \\
\chi & \longmapsto \boldsymbol{x}=\hat{\boldsymbol{\varphi}}(\chi, t), \quad \forall \chi \in \Omega_{\chi},
\end{aligned}
$$

onde $\chi$ é um ponto no domínio referencial. Tal transformação pode ser entendida como o movimento dos pontos da malha no domínio espacial. A velocidade de um ponto da malha especificada pela coordenada $\chi=\hat{\boldsymbol{\varphi}}^{-1}(\boldsymbol{x}, t)$ é então dada por

$$
\mathbf{v}^{*}(\boldsymbol{\chi}, t)=\mathbf{v}(\boldsymbol{x}, t)=\left.\frac{\partial \hat{\boldsymbol{\varphi}}}{\partial t}\right|_{\chi} .
$$

A derivada temporal de uma quantidade escalar física $\alpha^{*}(\boldsymbol{\chi}, t)=\alpha(\boldsymbol{x}, t)$ em um ponto da malha $\chi$, é dada por

$$
\left.\frac{\partial \alpha}{\partial t}\right|_{\chi}=\frac{\partial \alpha}{\partial t}+\mathbf{v} \cdot \nabla \alpha
$$

A relação entre os três referenciais pode ser vista na figura (2.1).

Para descrever as equações que governam um sistema mecânico na formulação ALE, é preciso expressar suas derivadas temporais em termos da derivada temporal no do- 
mínio de referência. Portanto, a derivada material (2.3), que está presente nas leis de conservação, descrita na formulação ALE, fica

$$
\frac{\mathrm{D} \alpha}{\mathrm{D} t}=\left.\frac{\partial \alpha}{\partial t}\right|_{\chi}+\mathbf{c} \cdot \nabla \alpha
$$

onde $\mathbf{c} \doteq \mathbf{u}-\mathbf{v}$ é a velocidade convectiva. A ideia dessa formulação é especificar a velocidade da malha $\mathbf{v}$ de modo que esta se mantenha com a melhor qualidade possível. Mais detalhes sobre a formulação ALE podem ser encontrados em Donea et al. [19].

No texto que se segue, a derivada material e a derivada temporal no domínio de referência serão denotadas por

$$
\left.\partial_{t}^{* *} \alpha \doteq \frac{\partial \alpha}{\partial t}\right|_{\boldsymbol{X}} \quad \text { e }\left.\quad \partial_{t}^{*} \alpha \doteq \frac{\partial \alpha}{\partial t}\right|_{\chi},
$$

respectivamente. Além disso, são escolhidos os domínios referencial e material de forma que

$$
\boldsymbol{\varphi}(\boldsymbol{X}, 0)=\left(\hat{\boldsymbol{\varphi}}^{-1} \circ \boldsymbol{\varphi}\right)(\boldsymbol{X}, 0)=i d_{\boldsymbol{X}}
$$

onde $i d_{\boldsymbol{X}}$ é a função identidade, ou seja, tais domínios coincidem com a configuração do fluido no instante inicial $t=0$.

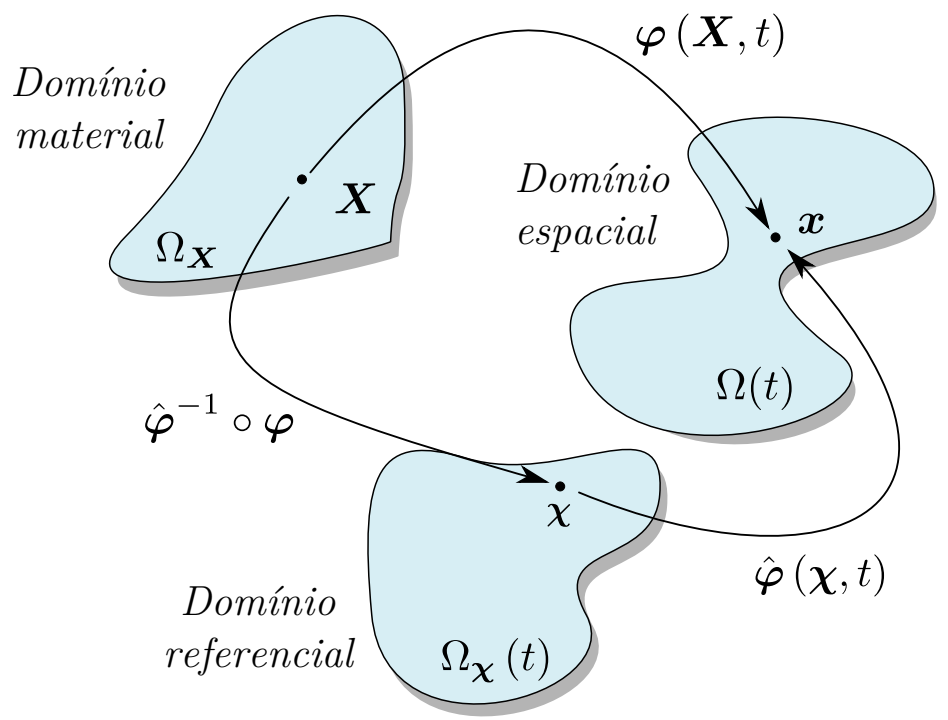

Figura 2.1: Esboço do mapeamento entre os domínios espacial, material e referencial. 


\subsection{Formulação variacional das equações de Navier-Stokes}

\subsubsection{Problema contínuo}

A fim de elucidar os métodos numéricos adotados neste trabalho com respeito ao método de elementos finitos na formulação ALE, desconsidere por ora os fenômenos de superfície. Considere, por enquanto, um escoamento monofásico e incompressível de um fluido Newtoniano cuja densidade $\rho$ e viscosidade $\mu$ são constantes ao longo de todo o domínio $\Omega \subset \mathbb{R}^{d}$ onde o fluido está confinado. A fronteira de $\Omega$, denotada por $\partial \Omega$ e não necessariamente fixa, divide-se em três partes: $\Gamma_{D}$, onde a velocidade é especificada completamente; $\Gamma_{N}$, onde a tração é especificada completamente; e $\Gamma_{M}$, onde a velocidade normal e a tração tangencial são especificadas. As equações diferenciais referentes a esse problema são dadas por

$$
\begin{aligned}
\rho\left[\partial_{t}^{*} \mathbf{u}+(\mathbf{c} \cdot \nabla) \mathbf{u}\right] & =\nabla \cdot \boldsymbol{\sigma}+\rho \boldsymbol{g}, & & \boldsymbol{x} \in \Omega, \\
\nabla \cdot \mathbf{u} & =0, & & \boldsymbol{x} \in \Omega,
\end{aligned}
$$

com as condições de contorno

$$
\begin{array}{rlrl}
\mathbf{u} & =\mathbf{u}_{\Gamma}, & & \boldsymbol{x} \in \Gamma_{D}, \\
\boldsymbol{\sigma} \cdot \mathbf{n} & =\boldsymbol{\sigma}_{n}, & & \boldsymbol{x} \in \Gamma_{N}, \\
(\mathbf{I}-\mathbf{n n}) \cdot \boldsymbol{\sigma} \cdot \mathbf{n}=\boldsymbol{\sigma}_{\tau}, & \boldsymbol{x} \in \Gamma_{M}, \\
\mathbf{u} \cdot \mathbf{n}=U_{n}, & \boldsymbol{x} \in \Gamma_{M},
\end{array}
$$

para todo tempo $t \in \mathbb{R}^{+}$, e com a condição inicial solenoidal:

$$
\mathbf{u}(\boldsymbol{x}, t=0)=\mathbf{u}_{0}(\boldsymbol{x}), \quad \text { em } \Omega
$$

onde o termo entre colchetes em (2.10a) é a derivada material de $\mathbf{u}$ na formulação ALE (cf. (2.7) e (2.8)), $\boldsymbol{\sigma}$ o tensor de tensões de Cauchy, $\mathbf{n}$ o versor normal à superfície apontando para fora do fluido, $\boldsymbol{\sigma}_{n}$ a tensão que o meio externo exerce sobre o fluido, $\boldsymbol{\sigma}_{\tau}$ a componente tangencial da tensão que o meio externo exerce sobre o fluido, $U_{n}$ a componente normal da velocidade, I o tensor identidade e $\boldsymbol{g}$ as forças de campo (e.g., gravidade). O tensor de tensões neste caso é dado por

$$
\boldsymbol{\sigma}=-p \mathbf{I}+2 \mu D \mathbf{u}
$$


onde $p$ é a pressão e $D \mathbf{u}$ é a de taxa de deformação dada por

$$
D \mathbf{u} \doteq \frac{1}{2}\left(\nabla \mathbf{u}+\nabla \mathbf{u}^{T}\right) .
$$

Introduzindo os espaços

$$
\begin{aligned}
& W_{0} \doteq\left\{\mathbf{w} \in\left(H^{1}(\Omega)\right)^{d} \mid \mathbf{w}=\mathbf{0} \text { em } \Gamma_{D}, \mathbf{w} \cdot \mathbf{n}=0 \text { em } \Gamma_{M}\right\} \\
& W_{u} \doteq\left\{\mathbf{w} \in\left(H^{1}(\Omega)\right)^{d} \mid \mathbf{w}=\mathbf{u}_{\Gamma} \text { em } \Gamma_{D}, \mathbf{w} \cdot \mathbf{n}=U_{n} \text { em } \Gamma_{M}\right\} \\
& Q \doteq L^{2}(\Omega) \quad\left(\text { ou } L^{2}(\Omega) / \mathbb{R} \text { se } \Gamma_{N}=\emptyset\right),
\end{aligned}
$$

onde $H^{1}(\Omega)$ e $L^{2}(\Omega)$ são os espaços de Sobolev usuais, a formulação variacional correspondente ao problema fica: encontrar $(\mathbf{u}, p) \in W_{u} \times Q$ tal que

$$
\begin{aligned}
& \left(\rho \partial_{t}^{*} \mathbf{u}, \boldsymbol{w}\right)+a(\mathbf{u}, \mathbf{w})+c(\mathbf{c} ; \mathbf{u}, \mathbf{w})-b(p, \mathbf{w})=\mathcal{P}(\mathbf{w}) \\
& -b(q, \mathbf{u})=0
\end{aligned}
$$

$\forall(\mathbf{w}, q) \in W_{0} \times Q$, onde

$$
\begin{aligned}
(\mathbf{u}, \mathbf{w}) & \doteq \int_{\Omega} \mathbf{u} \cdot \mathbf{w}, \\
a(\mathbf{u}, \mathbf{w}) & \doteq \int_{\Omega} 2 \mu D \mathbf{u}: D \mathbf{w}, \\
c(\mathbf{c} ; \mathbf{u}, \mathbf{w}) & \doteq \int_{\Omega} \rho[(\mathbf{c} \cdot \nabla) \mathbf{u}] \cdot \mathbf{w} \\
b(p, \mathbf{w}) & \doteq \int_{\Omega} p \nabla \cdot \mathbf{w}, \\
\mathcal{P}(\mathbf{w}) & \doteq(\rho \boldsymbol{g}, \mathbf{w})+\int_{\Gamma_{N}} \boldsymbol{\sigma}_{n} \cdot \mathbf{w}+\int_{\Gamma_{M}} \boldsymbol{\sigma}_{\tau} \cdot \mathbf{w},
\end{aligned}
$$

com a condição incial solenoidal $\mathbf{u}(\boldsymbol{x}, 0)=\mathbf{u}_{0}(\boldsymbol{x})$.

A velocidade $\mathbf{v} \in\left(H^{1}(\Omega)\right)^{d}$ é arbitrária e usualmente restrita à condição

$$
\mathbf{u} \cdot \mathbf{n}=\mathbf{v} \cdot \mathbf{n}, \quad \text { em } \partial \Omega
$$

à qual garante que a fronteira da malha seja conforme com a do domínio a todo instante. Há casos, porém, em que é assumida a possibilidade de partículas do fluido que estejam na fronteira migrarem para o interior do domínio, de modo que a relação (2.18) não mais garanta esta conformidade entre malha e domínio. Entretanto, considerar esta situação não está no escopo deste capítulo. Outra observação com respeito à velocidade da malha $\mathbf{v}$ no problema contínuo é que, apesar de sua arbitrariedade, ela é também uma incógnita, uma vez que, em geral, $\mathbf{u} \cdot \mathbf{n}$ não é conhecido em toda a fronteira $\partial \Omega$. 
A seguir, algumas notações são introduzidas à medida que as estratégias de discretização espacial adotadas são apresentadas.

\subsubsection{Discretização espacial}

Para uma partição $\mathcal{T}^{h}$ do domínio $\Omega$ representado pela malha computacional, considere a aproximação de Galerkin obtida restringindo a forma fraca (2.16) para os espaços de dimensão finita $W_{0, h} \subset W_{0}, W_{u, h} \subset W_{u}$ e $Q_{h} \subset Q$, cujos vetores são polinômios em cada elemento simplicial* $K \in \mathcal{T}^{h}$. Para o texto que se segue, nós da malha referem-se tanto aos pontos no encontro de semi-retas como aos pontos associados às arestas, às faces, etc..

Considere $\eta=\left\{1, \ldots, n_{u}\right\}$ o conjunto indicial dos nós de velocidade na malha de elementos finitos, e $\hat{\eta}=\left\{1, \ldots, n_{p}\right\}$ o conjunto indicial dos nós de pressão, onde em geral $\eta \neq \hat{\eta}$. Será denotado por $\eta_{D} \subset \eta$ o conjunto dos nós da velocidade que estão na porção do contorno Dirichlet. A aproximação da velocidade, $\mathbf{u}_{h}$, e a aproximação da pressão, $p_{h}$, são escritos como

$$
\begin{aligned}
\mathbf{u}_{h}(\boldsymbol{x}, t) & =\sum_{j \in \eta} \phi^{j}(\boldsymbol{x}, t) \mathbf{u}^{j}(t), \\
p_{h}(\boldsymbol{x}, t) & =\sum_{k \in \hat{\eta}} \psi^{k}(\boldsymbol{x}, t) p^{k}(t),
\end{aligned}
$$

onde $\phi^{j}$ e $\psi^{k}$ são as funções de interpolação usuais associadas ao índices globais do nó $j$ e $k$, respectivamente (satisfazendo as propriedades delta e partição de unidade), e $\mathbf{u}^{j}(t)$ e $p^{k}(t)$ são coeficientes de interpolação. A dependência temporal de $\phi^{j}$ e $\psi^{k}$ é pelo fato de que tais funções são construídas de acordo com as posições nodais da malha as quais não são fixas no espaço, além disso,

$$
\frac{\partial \phi^{j}}{\partial t}=-\mathbf{v} \cdot \nabla \phi^{j},\left.\quad \frac{\partial \phi^{j}}{\partial t}\right|_{\chi}=0 .
$$

A malha $\mathcal{T}^{h}$ é a interpolação da geometria de $\Omega$, ou seja, a interpolação das posições $\mathbf{x}^{k}$ de seus nós, com $k \in \eta$. Tal interpolação é aqui feita de forma isoparamétrica com respeito às funções interpoladoras da velocidade, i.e.,

$$
\mathbf{x}_{h}(\boldsymbol{x}, t)=\sum_{j \in \eta} \vartheta^{j}(\boldsymbol{x}, t) \mathbf{x}^{j}(t), \quad \vartheta^{j}=\phi^{j}
$$

${ }^{*}$ Triângulo se $d=2$ e tetraedro se $d=3$. 
que sugere a seguinte aproximação $\mathbf{v}_{h}$ para a velocidade da malha:

$$
\mathbf{v}_{h}(\boldsymbol{x}, t)=\left.\frac{\partial \mathbf{x}}{\partial t}\right|_{\chi}=\sum_{j \in \eta} \vartheta^{j}(\boldsymbol{x}, t) \mathbf{v}^{j}(t) .
$$

Observação 2.1: Não confundir a variável independente $\boldsymbol{x}$ com a posição da malha $\mathbf{x}_{h}$ e com as posições nodais $\mathbf{x}^{j}$. Note também que $\mathbf{x}_{h}(\boldsymbol{x}, t)=\boldsymbol{x}$. Esta propriedade pode ser usada para definir um mapeamento de um elemento unitário para um elemento genérico da malha, pois, definindo um mapeamento $\boldsymbol{x}=\boldsymbol{\Phi}(\boldsymbol{y})$ em cada elemento, este pode ser o dado por

$$
\mathbf{x}_{h}(\boldsymbol{\Phi}(\boldsymbol{y}), t) \equiv \sum_{j} \hat{\vartheta}^{j}(\boldsymbol{y}) \mathbf{x}^{j}(t)=\boldsymbol{\Phi}(\boldsymbol{y})
$$

já que $\hat{\vartheta}^{j}(\boldsymbol{y})=\vartheta^{j}(\boldsymbol{\Phi}(\boldsymbol{y}))$ é conhecido a priori.

A versão discretizada espacialmente do problema variacional (2.16) é então dada por: encontrar $\left(\mathbf{u}_{h}, p_{h}\right) \in W_{u, h} \times Q_{h}$ tal que

$$
\begin{aligned}
& \left(\rho \partial_{t}^{*} \mathbf{u}_{h}, \mathbf{w}_{h}\right)+a\left(\mathbf{u}_{h}, \mathbf{w}_{h}\right)+c\left(\mathbf{c}_{h} ; \mathbf{u}_{h}, \mathbf{w}_{h}\right)-b\left(p_{h}, \mathbf{w}_{h}\right)=\mathcal{P}\left(\mathbf{w}_{h}\right) \\
& -b\left(q_{h}, \mathbf{u}_{h}\right)=0
\end{aligned}
$$

para todo $\left(\mathbf{w}_{h}, q_{h}\right) \in W_{0, h} \times Q_{h}$, com uma condição inicial adequada.

Como é bem conhecido na literatura, a estabilidade do método depende da escolha do par de espaços $W_{u, h}$ e $Q_{h}$ quando se faz uso do método tradicional de Galerkin. Para um problema de Stokes, estes espaços estão relacionados pela condição necessária de Ladyzhenskaya-Babuška-Brezzi (LBB), ou condição inf-sup, expressa na seguinte forma:

$$
\inf _{q_{h} \in q_{h} \sup _{h} \in W_{0, h}} \frac{\left(q_{h}, \nabla \cdot \mathbf{w}_{h}\right)}{\left\|q_{h}\right\|_{0}\left\|\mathbf{w}_{h}\right\|_{1}} \geq \alpha>0
$$

onde $\alpha$ é uma constante. Se o par velocidade-pressão satisfaz essa condição, então a existência e unicidade estão garantidas e vale a seguinte estimativa para o erro

$$
\left\|\mathbf{u}-\mathbf{u}_{h}\right\|_{W_{u}}+\left\|p-p_{h}\right\|_{Q} \leq C\left(\inf _{\mathbf{w}_{h} \in W_{u, h}}\left\|\mathbf{u}-\mathbf{w}_{h}\right\|_{W_{u}}+\inf _{q_{h} \in Q_{h}}\left\|p-q_{h}\right\|_{Q}\right) .
$$

Uma lista com vários tipos de elementos que satisfazem essa condição (mini-elemento, elementos Taylor-Hood, etc.) pode ser encontrada em Gresho e Sani [27], enquanto que uma discussão sobre a condição de LBB pode ser encontrada em Brezzi e Fortin [8] e Brezzi et al. [7].

Uma forma de se contornar a condição inf-sup são as formulações estabilizadas, 
nas quais são feitas modificações na formulação variacional de modo a possibilitar a escolha de pares de velocidade-pressão que não seriam estáveis na formulação de Galerkin tradicional, como os de ordens iguais $P_{n} / P_{n}$.

Ambas as abordagens (Galerkin com pares satisfazendo inf-sup e formulações estabilizadas) são consideradas. O pares velocidade-pressão estudados são apresentados após a descrição da discretização temporal de (2.24).

\subsubsection{Discretização temporal}

A discretização temporal do problema na formulação ALE requer atenção para a velocidade da malha $\mathbf{v}_{h}$, uma vez que ela deve respeitar a evolução temporal da fronteira do domínio $\partial \Omega(t)$ (desconhecida em geral) e deve ser tal que otimize a qualidade da malha. Além disso, alguns autores recomendam que a formulação ALE discretizada satisfaça uma condição chamada de lei de conservação de geometria (GCL, geometric conservation law), que requer que um escoamento constante seja reproduzido exatamente pelo esquema numérico independentemente do movimento da malha (Guillard e Farhat [28]). Em Formaggia [23], é mostrado em um modelo de advecção-difusão linear que a GCL não é uma condição nem suficiente e nem necessária para estabilidade, mas que pode ajudar na precisão e, em casos particulares como o esquema de Euler implícito, aumentar a estabilidade. Investigações da GCL para as equações de Navier-Stokes no contexto de interação fluido-estrutura podem ser encontradas em Nobile [36].

Felizmente, a forma em (2.24a), dita forma não conservativa, satisfaz automaticamente a GCL, pois em um escoamento constante o termo convectivo, que contém o termo $\nabla \mathbf{u}_{h}$, anula-se, de modo que a velocidade da malha $\mathbf{v}_{h}$ não tenha efeito na equação. O cenário é diferente para a forma conservativa, que será apenas brevemente discutida. Tal forma é obtida pela substituição do termo $\left(\rho \partial_{t}^{*} \mathbf{u}_{h}, \mathbf{w}_{h}\right)$ em (2.24) por um equivalente obtido com o teorema de transporte de Leibnitz, i.e.,

$$
\frac{\mathrm{d}}{\mathrm{d} t}\left(\rho \mathbf{u}_{h}, \mathbf{w}_{h}\right)-\left(\left(\nabla \cdot \mathbf{v}_{h}\right) \rho \mathbf{u}_{h}, \mathbf{w}_{h}\right)
$$

antes de se fazer a discretização temporal. A desvantagem desta forma é que ela depende da escolha da discretização temporal e da velocidade da malha para que a GCL seja satisfeita. Mais especificamente, um esquema satisfaz a GCL, se a relação

$$
\int_{\Omega^{n+1}} \mathbf{w}_{h}-\int_{\Omega^{n}} \mathbf{w}_{h}=\mathrm{INT}_{t^{n}}^{t^{n+1}}\left[\int_{\Omega(t)} \nabla \cdot \mathbf{v}_{h} \mathbf{w}_{h}\right] \quad \forall \mathbf{w}_{h} \in W_{0, h},
$$

é satisfeita em cada intervalo de tempo pelo método de integração temporal $\operatorname{INT}_{t^{n}}^{t^{n+1}}$ adotado (Nobile [36]). Em Formaggia e Nobile [24] é feita a seguinte proposição: 
Proposição 2.1: Uma condição suficiente para que (2.28) se satisfaça é usar um esquema de integração no tempo para o termo ALE de grau $d \cdot s-1$, onde $d$ é a dimensão do espaço e s é o grau do polinômio usado para representar a evolução do deslocamento nodal em cada intervalo de tempo.

Outra forma de satisfazer a GCL na forma conservativa, apresentada em Étienne et al. [22], é, em vez de usar a velocidade da malha para calcular o termo $\nabla \cdot \mathbf{v}_{h}$ (i.e., calcular explicitamente o divergente da velocidade da malha discretizada $\mathbf{v}_{h}$ ), troca-se tal termo por outro usando a bem conhecida relação da mecânica do contínuo (ver, e.g., [41])

$$
\nabla \cdot \mathbf{v}(\boldsymbol{x}, t)=\left[\left.\frac{1}{J} \frac{\partial J}{\partial t}\right|_{\boldsymbol{X}}\right] \circ \boldsymbol{\varphi}^{-1}
$$

onde $J=J(\boldsymbol{X}, t), \boldsymbol{X} \in \Omega(0)$, é o determinante do gradiente da transformação $\boldsymbol{\varphi}(\boldsymbol{X}, t)$ de $\Omega(0)$ para $\Omega(t)$. Ou seja, o método se trata de trocar $\nabla \cdot \mathbf{v}_{h}$ pela versão discretizada do termo do lado direito de (2.29).

Neste trabalho, será usada a forma não conservativa com o esquema de segunda ordem de Crank-Nicolson, e será mostrado por exemplos numéricos que este esquema aplicado ao ALE mantém a alta ordem desde que a formulação variacional seja integrada no domínio correto.

Por simplicidade, será considerada uma interpolação linear no tempo para o movimento da malha em cada intervalo $\left[t^{n}, t^{n+1}\right]$ dada por

$$
\mathbf{x}_{h}(\boldsymbol{x}, t)=\sum_{j} \vartheta^{j}(\boldsymbol{x}, t)\left[\mathbf{x}^{j}\left(t^{n+1}\right) \frac{t-t^{n}}{\Delta t}+\mathbf{x}^{j}\left(t^{n}\right) \frac{t^{n+1}-t}{\Delta t}\right],
$$

e consequentemente pela expressão (2.22) a velocidade da malha fica

$$
\mathbf{v}_{h}(\boldsymbol{x}, t)=\sum_{j} \vartheta^{j}(\boldsymbol{x}, t) \frac{\mathbf{x}^{j}\left(t^{n+1}\right)-\mathbf{x}^{j}\left(t^{n}\right)}{\Delta t} .
$$

Em Nobile [36] também são apresentadas expressões no caso de interpolações com polinômios quadráticos, assim como seu impacto na precisão do método. Denotando por $\underline{\mathbf{x}}$ e $\underline{\mathbf{v}}$ as matrizes em $\mathbb{M}_{d, n_{u}}(\mathbb{R})$ (matrizes com dimensão $d$ por $n_{u}$ ) dos coeficientes das funções $\mathbf{x}_{h}$ e $\mathbf{v}_{h}$, respectivamente, tal que $\left.\underline{\mathbf{x}}\right|_{i, j}=\mathbf{x}^{j} \cdot \mathbf{e}^{i}$ e $\left.\underline{\mathbf{v}}\right|_{i, j}=\mathbf{v}^{j} \cdot \mathbf{e}^{i}$, é possível 
reescrever as Eqs. (2.30) e (2.31) na forma mais prática

$$
\begin{aligned}
& \underline{\mathbf{x}}(t)=\underline{\mathbf{x}}^{n+1} \frac{t-t^{n}}{\Delta t}+\underline{\mathbf{x}}^{n} \frac{t^{n+1}-t}{\Delta t} \\
& \underline{\mathbf{v}}(t)=\frac{1}{\Delta t}\left(\underline{\mathbf{x}}^{n+1}-\underline{\mathbf{x}}^{n}\right), \quad t \in\left[t^{n}, t^{n+1}\right] .
\end{aligned}
$$

onde $\underline{\mathbf{x}}^{n+\theta} \doteq \underline{\mathbf{x}}\left(t^{n+\theta}\right), 0 \leq \theta \leq 1$.

Observação 2.2: Optou-se por trabalhar com os coeficientes em vez das próprias funções $\mathbf{x}_{h}^{n+1}(\boldsymbol{x}) \doteq \mathbf{x}_{h}\left(\boldsymbol{x}, t^{n+1}\right)$ e $\mathbf{x}_{h}^{n}(\boldsymbol{x}) \doteq \mathbf{x}_{h}\left(\boldsymbol{x}, t^{n}\right)$ pois estas têm domínios diferentes entre si, já que a primeira está definida em $\Omega^{n}$ e a outra em $\Omega^{n+1}$, portanto, não faz sentido definir uma função $\mathbf{x}_{h}(\boldsymbol{x}, t)$ como combinação dessas duas.

Observação 2.3: De (2.32b), observa-se que a velocidade da malha $\mathbf{v}_{h}$ é constante em cada nó em cada intervalo, e por isso, uma vez que $\mathbf{v}_{h}$ é descontínua em todos os instantes $t^{n}$, é escolhido $\mathbf{v}_{h}\left(\boldsymbol{x}, t^{n}\right)=\lim _{t \rightarrow t^{n}-} \mathbf{v}_{h}(\boldsymbol{x}, t)$ quando necessário.

O método de Crank-Nicolson, aplicado em uma equação do tipo $\dot{u}(t)=f(t)$, $u(t), t \in \mathbb{R}$, consiste em avaliar ou aproximar a derivada $f$ no tempo $t^{n+1 / 2}=\left(t^{n+1}+\right.$ $\left.t^{n}\right) / 2$,

$$
\frac{1}{\Delta t}\left(u^{n+1}-u^{n}\right) \simeq f^{n+1 / 2} \simeq\left(f^{n+1}+f^{n}\right) / 2 .
$$

A primeira "variável" a ser integrada no tempo é o domínio $\Omega(t)$, determinado pela função $\mathbf{x}_{h}$. Utilizando Crank-Nicolson, tem-se

$$
\begin{aligned}
& \Delta t^{-1} \operatorname{INT}_{t^{n}}^{t^{n+1}}\left[\mathbf{x}_{h}(\boldsymbol{x}, t)\right]=\mathbf{x}_{h}\left(\boldsymbol{x}, t^{n+1 / 2}\right), \\
& \Delta t^{-1} \operatorname{INT}_{t^{n}}^{t^{n+1}}[\Omega(t)]=\Omega^{n+1 / 2}
\end{aligned}
$$

que na prática, implica em computar a malha com os nós $\underline{\mathbf{x}}^{n+1 / 2} \doteq\left(\underline{\mathbf{x}}^{n+1}+\underline{\mathbf{x}}^{n}\right) / 2$.

Seja $\underline{\phi}(\boldsymbol{x}, t)$ um vetor em $\mathbb{R}^{\eta}$ tal que seu $i$-ésimo elemento seja a $i$-ésima função da base de $\mathbf{u}_{h}$, ou seja, $\left.\underline{\phi}(\boldsymbol{x}, t)\right|_{i}=\phi^{i}(\boldsymbol{x}, t)$, e seja $\underline{\mathbf{u}}(t)$ uma matrix em $\mathbb{M}_{d, n_{U}}(\mathbb{R})$ com os coeficientes de $\mathbf{u}_{h}$, de modo que $\mathbf{u}_{h}(\boldsymbol{x}, t)=\underline{\mathbf{u}}(t) \cdot \underline{\phi}(\boldsymbol{x}, t)$. Da segunda propriedade de (2.20), tem-se que

$$
\partial_{t}^{*} \mathbf{u}_{h}=\frac{\mathrm{d} \underline{\mathbf{u}}}{\mathrm{d} t} \cdot \underline{\phi} \doteq \mathrm{d}_{t} \underline{\mathbf{u}} \cdot \underline{\phi} .
$$

$\mathrm{Na}$ integração temporal, o primeiro termo de (2.24), no método de Crank-Nicolson, 
fica

$$
\Delta t^{-1} \mathrm{INT}_{t^{n}}^{t^{n+1}}\left[\int_{\Omega(t)} \rho\left(\mathrm{d}_{t} \underline{\mathbf{u}} \cdot \underline{\phi}\right) \cdot \mathbf{w}_{h}\right]=\int_{\Omega^{n+1 / 2}} \rho \delta_{t} \mathbf{u}_{h} \cdot \mathbf{w}_{h},
$$

onde

$$
\delta_{t} \mathbf{u}_{h}(\boldsymbol{x}) \doteq \Delta t^{-1}\left(\underline{\mathbf{u}}^{n+1}-\underline{\mathbf{u}}^{n}\right) \cdot \underline{\phi}^{n+1 / 2}(\boldsymbol{x})
$$

e onde, seguindo a notação, $\underline{\mathbf{u}}^{n+\theta} \doteq \underline{\mathbf{u}}\left(t^{n+\theta}\right)$ e $\underline{\phi}^{n+\theta}(\boldsymbol{x}) \doteq \underline{\phi}\left(\boldsymbol{x}, t^{n+\theta}\right), 0 \leq \theta \leq 1$. O gradiente de $\nabla \mathbf{u}_{h}$, em termos de seus coeficientes, é dado por

$$
\nabla \mathbf{u}_{h}(\boldsymbol{x}, t)=\underline{\mathbf{u}}(t) \cdot \nabla \underline{\phi}(\boldsymbol{x}, t),
$$

onde o gradiente atua em cada componente do vetor $\underline{\phi}$, de forma que $\nabla \underline{\phi}(\boldsymbol{x}, t) \in$ $\mathbb{M}_{\eta, d}(\mathbb{R})$. O termo $\underline{\mathbf{u}}(t)$ avaliado no tempo $t^{n+1 / 2}$, será aproximado como

$$
\underline{\mathbf{u}}\left(t^{n+1 / 2}\right) \simeq\left(\underline{\mathbf{u}}^{n+1}+\underline{\mathbf{u}}^{n}\right) / 2 .
$$

A expressão para o gradiente simétrico $D \mathbf{u}_{h}$ é análoga. Com isso, a integração temporal dos termos restantes do lado direito de (2.24), fica

$$
\begin{aligned}
& \Delta t^{-1} \mathrm{INT}_{t^{n}}^{t^{n+1}}\left[\int_{\Omega(t)} \nabla \mathbf{u}_{h} \cdot \mathbf{c}_{h} \cdot \mathbf{w}_{h}+\left(-\mathbf{I}_{d} p_{h}+2 \mu D \mathbf{u}_{h}\right): D \mathbf{w}_{h}\right]= \\
& \quad=\left[\int_{\Omega^{n+1 / 2}} \nabla \mathbf{u}_{h}^{n+1 / 2} \cdot \mathbf{c}_{h}^{n+1 / 2} \cdot \mathbf{w}_{h}+\left(-\mathbf{I}_{d} p_{h}^{n+1 / 2}+2 \mu D \mathbf{u}_{h}^{n+1 / 2}\right): D \mathbf{w}_{h}\right],
\end{aligned}
$$

onde

$$
\begin{aligned}
\nabla \mathbf{u}_{h}^{n+1 / 2}(\boldsymbol{x}) & \doteq \underline{\mathbf{u}}^{n+1 / 2} \cdot \nabla \underline{\phi}^{n+1 / 2}(\boldsymbol{x}), \\
\mathbf{c}_{h}^{n+1 / 2}(\boldsymbol{x}) & \doteq \underline{\mathbf{u}}^{n+1 / 2} \cdot \underline{\phi}^{n+1 / 2}(\boldsymbol{x})-\mathbf{v}_{h}\left(\boldsymbol{x}, t^{n+1 / 2}\right), \\
p_{h}^{n+1 / 2}(\boldsymbol{x}) & \doteq\left(\underline{p}^{n+1 / 2}\right)^{T} \cdot \underline{\psi}^{n+1 / 2}(\boldsymbol{x}),
\end{aligned}
$$

e onde $\underline{p}, \underline{\psi} \in \mathbb{M}_{n_{p}, 1}(\mathbb{R})$ são definições análogas às de $\underline{\mathbf{u}}$ e $\underline{\phi}$, só que para a pressão.

Finalmente,

$$
\begin{aligned}
\int_{\Omega^{n+1 / 2}}\left[\rho\left(\delta_{t} \mathbf{u}_{h}^{n}+\nabla \mathbf{u}_{h}^{n+1 / 2} \cdot \mathbf{c}_{h}^{n+1 / 2}\right) \cdot \mathbf{w}_{h}+\right. & \\
& \left.+\left(-\mathbf{I}_{d} p_{h}^{n+1}+2 \mu D \mathbf{u}_{h}^{n+1 / 2}\right): D \mathbf{w}_{h}\right]=\mathcal{P}^{n+1 / 2}\left(\mathbf{w}_{h}\right), \\
-\int_{\Omega^{n+1 / 2}} q_{h} \nabla \cdot \mathbf{u}_{h}^{n+1 / 2}= & 0,
\end{aligned}
$$

onde a pressão $p_{h}^{n+1 / 2}$ foi trocada por $p_{h}^{n+1} \doteq\left(\underline{p}^{n+1}\right)^{T} \cdot \underline{\psi}^{n+1 / 2}(\boldsymbol{x})$, pois os resultados 
apontam que dessa forma o erro é ligeiramente reduzido. Deve-se lembrar que no problema (2.42) o domínio $\Omega^{n+1 / 2}$ também é uma incógnita, pois não se conhecem as posições $\underline{x}^{n+1}$ no bordo (que ao contrário das posições no interior, não são arbitrárias) e resolver todo o problema de forma monolítica é muito custoso. A versão discretizada da restrição que acopla a velocidade da malha e o domínio (cf. (2.18)) pode ser escrita como

$$
\sum_{i}\left[\underline{\mathbf{u}}^{n+1 / 2}-\underline{\mathbf{v}}^{n+1 / 2}\right]_{i, j}\left[\underline{\mathbf{n}}^{n+1 / 2}\right]_{i, j}=0, \quad \text { para cada nó } j \in \eta,
$$

onde $\underline{\mathbf{n}}^{n+1 / 2} \in \mathbb{M}_{d, n_{u}}(\mathbb{R})$ é a matriz com os coeficientes da normal em $t^{n+1 / 2}$. A primeira dificuldade da equação acima é definir uma normal em cada nó $k$, que será investigado mais adiante. Considere como primeiro passo que sejam escolhidos movimentos totalmente lagrangeanos para uma malha auxiliar $\underline{\mathbf{x}}_{a u x}^{n+1}$ (para que a velocidade da malha satisfaça (2.43) automaticamente). Então

$$
\underline{\mathbf{x}}_{a u x}^{n+1}=\underline{\mathbf{x}}^{n}+\Delta t \underline{\mathbf{u}}^{n+1 / 2} \text {. }
$$

Em vez de usar $\underline{\mathbf{x}}_{\text {aux }}^{n+1}$ como a malha definitiva e resolver implicitamente a equação anterior (já que $\underline{\mathbf{u}}^{n+1 / 2}$ não é conhecida) junto com as equações de movimento (é o mesmo que resolver com lagrangeano puro), é feito o seguinte procedimento. Calculase uma extrapolação para a velocidade intermediária:

$$
\underline{\mathbf{u}}_{e x t}^{n+1 / 2} \simeq(3 / 2) \underline{\mathbf{u}}^{n}-(1 / 2) \underline{\mathbf{u}}^{n-1},
$$

e então, substituindo $\underline{\mathbf{u}}^{n+1 / 2}$ por $\underline{\mathbf{u}}_{\text {ext }}^{n+1 / 2}$ em (2.44), chega-se em

$$
\underline{\mathbf{x}}_{\text {aux }}^{n+1}=\underline{\mathbf{x}}^{n}+\frac{\Delta t}{2}\left(3 \underline{\mathbf{u}}^{n}-\underline{\mathbf{u}}^{n-1}\right)
$$

onde é recuperado o método de segunda ordem Adams-Bashforth para a integração da equação $d_{t} \underline{\mathbf{x}}=\underline{\mathbf{u}}$. A extrapolação feita restringe as condições de estabilidade as quais não serão investigadas aqui, mas são discutidas em Nobile [36] em outro contexto. Em seguida, como os pontos internos podem ser movidos arbitrariamente, otimiza-se a qualidade da malha auxiliar $\underline{\mathbf{x}}_{a u x}^{n+1}$ e obtém-se uma malha definitiva $\underline{\mathbf{x}}^{n+1}$.

O procedimento completo feito em cada passo de tempo pode ser visto no algoritmo $(2.1)$. 


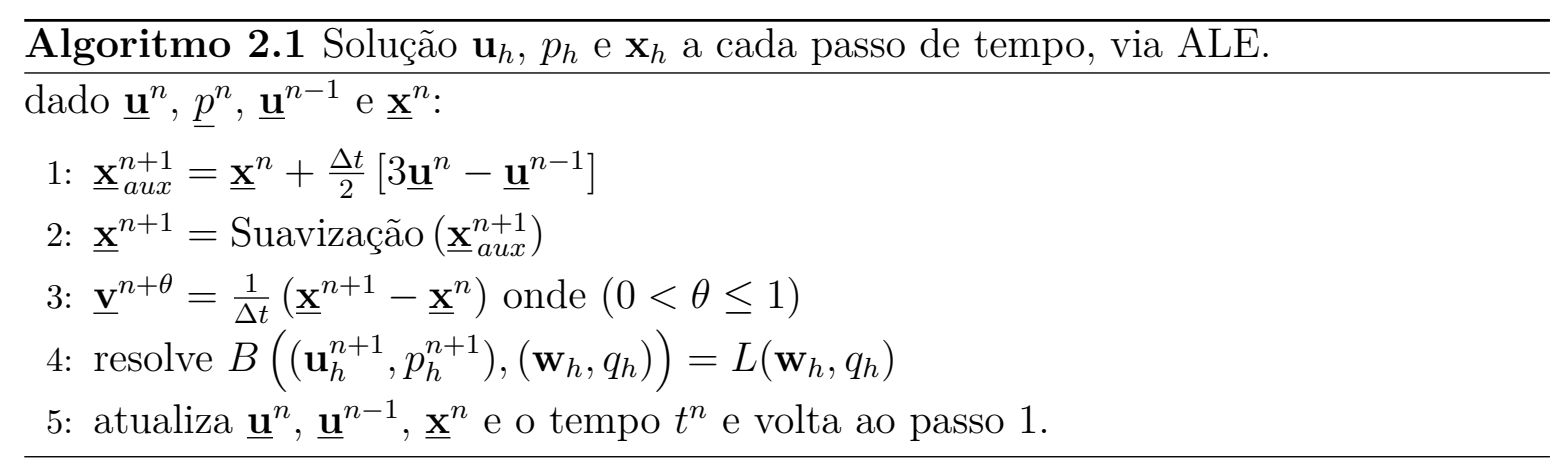

Seguem algumas justificativas e observações sobre o algoritmo:

- No passo 1, uma malha intermediária $\underline{\mathbf{x}}_{a u x}^{n+1}$ é obtida pelo movimento puramente lagrangeano tanto dos pontos do bordo como dos pontos interiores.

- No passo 2, a malha definitiva $\underline{\mathbf{x}}^{n+1}$ é obtida por meio de modificações na malha intermediária que visam melhorar a qualidade da malha preservando sua forma original. Para isso, apenas os pontos interiores são modificados e, em alguns casos, os pontos do bordo são movidos tangencialmente.

- Ainda no passo 2, é importante entender que, como $\underline{x}^{n+1}$ foi obtida por meio de uma extrapolação da velocidade, então as equações em (2.42) estão sendo integradas em um domínio $\tilde{\Omega}^{n+1 / 2}$ aproximado do domínio correto $\Omega^{n+1 / 2}$.

- É possível estender o algoritmo para uma estratégia preditor-corretor, onde $\underline{x}^{n+1}$ é corrigido a partir da solução encontrada $\underline{\mathbf{u}}^{n+1}$. Para isso, definem-se $\underline{\mathbf{x}}^{n+1,(k)} \mathrm{e}$ $\underline{\mathbf{u}}^{n+1,(k)}$, e os passo de 1 a 4 são repetidos até que

$$
\left\|\left\{\underline{\mathbf{x}}^{n+1,(k+1)}, \underline{\mathbf{u}}^{n+1,(k+1)}\right\}-\left\{\underline{\mathbf{x}}^{n+1,(k)}, \underline{\mathbf{u}}^{n+1,(k)}\right\}\right\|<\varepsilon,
$$

ou algum outro critério de parada seja satisfeito, ou por um número fixo de iterações. Também é possível inserir os passos 1-3 dentro das iterações de Newton dos sistemas de equações não lineares no passo 4 .

- Como Adams-Bashforth não é auto-inicializante, é feito $\underline{\mathbf{x}}_{a u x}^{1}=\underline{\mathbf{x}}^{0}+\Delta t \underline{\mathbf{u}}^{0}$ no passo 1 do algoritmo e adotada a estratégia de preditor corretor descrito no item anterior, com um número fixo de iterações igual 10.

- Resultados utilizando Euler implícito de segunda ordem em vez de Crank-Nicolson para o passo 4 pode ser encontrado em Förster et al. [25]. 


\subsubsection{Sistema de equações}

Apenas com a finalidade de introduzir algumas notações utilizadas mais adiante, é apresentado nesta subseção o sistema de equações não lineares no tempo $t^{n+1}$ da formulação variacional discreta (2.42) em uma forma mais conveniente para a implementação.

As $d$ equações do momento linear de cada nó de velocidade $i$ são obtidas escolhendose na equação (2.42a) $\mathbf{w}_{h}=\phi^{i} \mathbf{e}^{m}, m=1, \ldots, d$, onde $\mathbf{e}^{1}, . ., \mathbf{e}^{d}$ são os versores da base canônica. Tais equações podem ser escritas na forma residual:

$$
\int_{\Omega^{n+\theta}} \rho \delta_{t} \mathbf{u}_{h}^{n} \phi^{i}+\ldots-\mathcal{P}^{n+\theta}\left(\phi^{i}\right) \doteq \mathcal{F}_{u}\left(\mathbf{u}_{h}, p_{h}, \phi^{i}\right)=\mathbf{0} .
$$

Com isso, o vetor $\mathcal{F}_{u}\left(\mathbf{u}_{h}, p_{h}, \phi^{i}\right)$ é dito o resíduo do momento linear do nó $i$. O índice inferior $u$ em $\mathcal{F}$ é para indicar que o resíduo $\mathcal{F}_{u}$ corresponde às equações de momento linear. O forma $\mathcal{P}^{n+\theta}\left(\phi^{i}\right)$ significa

$$
\mathcal{P}^{n+\theta}\left(\phi^{i}\right)=\left[\mathcal{P}^{n+\theta}\left(\phi^{i} \mathbf{e}^{1}\right), \ldots, \mathcal{P}^{n+\theta}\left(\phi^{i} \mathbf{e}^{d}\right)\right]^{T},
$$

e analogamente para as formas compactas $(\cdot, \cdot), a(\cdot, \cdot), b(\cdot, \cdot)$ e $c(\cdot ; \cdot, \cdot)$.

O resíduo da incompressiblidade do nó de pressão $k$ é definido como

$$
-\int_{\Omega^{n+\theta}} \psi^{k} \nabla \cdot \mathbf{u}_{h}^{n+\theta} \doteq \mathcal{F}_{p}\left(\mathbf{u}_{h}, p_{h}, \psi^{k}\right)=0
$$

onde agora o índice inferior $p$ em $\mathcal{F}$ é para indicar que o resíduo $\mathcal{F}_{p}$ corresponde às equações de incompressiblidade.

A letra $\mathcal{F}$, sem especificação do índice $u$ ou $p$, referir-se-á ao vetor global de resíduos, enquanto a letra $\mathcal{J}$ referir-se-á ao jacobiano associado ao resíduo $\mathcal{F}$, ou seja,

$$
\mathcal{J}=\frac{\partial \mathcal{F}}{\partial(\mathbf{u}, p)}
$$

Dessa forma, o método de Newton aplicado às equações em questão é escrito como

$$
\mathcal{J}^{k}\left[\begin{array}{c}
\Delta \underline{\mathbf{u}} \\
\Delta \underline{p}
\end{array}\right]=-\mathcal{F}^{k},
$$

onde $\underline{\mathbf{u}}$ e $\underline{p}$ denotam os coeficientes da velocidade e da pressão, respectivamente.

\subsection{Elementos adotados}

Quatro tipos de pares de elementos são considerados em testes de precisão, desempenho e facilidade de implementação a fim de auxiliar na escolha de um elemento em futuras implementações. A seguir, é feita uma breve descrição de cada um deles e ao final do 
capítulo são apresentados os testes numéricos.

\section{Elemento $P_{1} / P_{1}$ estabilizado com GLS}

O método Galerkin/Least-squares (GLS) foi proposto para equações de advecçãodifusão em Hughes e Franca [31]. Definindo a forma bilinear e linear associadas à formulação variacional de Galerkin do problema por $B(\cdot, \cdot)$ e $L(\cdot, \cdot)$, respectivamente, a formulação em (2.24), graças à arbitrariedade de $\mathbf{w}_{h}$ e $p_{h}$, pode ser reescrita como

$$
B\left(\left(\mathbf{u}_{h}, p_{h}\right),\left(\mathbf{w}_{h}, q_{h}\right)\right)=L\left(\mathbf{w}_{h}, q_{h}\right), \quad \forall\left(\mathbf{w}_{h}, q_{h}\right) \in W_{0, h} \times Q_{h} .
$$

O método GLS consiste em modificar a forma fraca (2.52) adicionando termos provenientes da minimização de um resíduo. Com isso, a forma bilinear estabilizada $B_{S}(\cdot, \cdot)$ deste problema fica:

$$
\begin{aligned}
B_{S}\left(\left(\mathbf{u}_{h}, p_{h}\right),\left(\mathbf{w}_{h}, q_{h}\right)\right) & \doteq B\left(\left(\mathbf{u}_{h}, p_{h}\right),\left(\mathbf{w}_{h}, q_{h}\right)\right)+ \\
& +\sum_{K}\left[\tau_{k}\left(\rho \mathbf{c}_{h} \cdot \nabla \mathbf{w}_{h}+\nabla q_{h}, \mathbf{R}_{h}^{K}\right)_{K}+\delta_{K}\left(\nabla \cdot \mathbf{u}_{h}, \nabla \cdot \mathbf{w}_{h}\right)_{K}\right]
\end{aligned}
$$

onde

$$
\mathbf{R}_{h}^{K} \doteq \rho\left[\partial_{t}^{*} \mathbf{u}_{h}+\left(\mathbf{c}_{h} \cdot \nabla\right) \mathbf{u}_{h}\right]+\nabla p_{h}-\rho \mathbf{g}, \quad \boldsymbol{x} \in K
$$

Uma escolha para os parâmetros $\tau_{K}$ e $\delta_{K}$ pode ser encontrada em Codina [13] no contexto de outro método, o ASGS (algebraic subgrid scale), que coincide com o GLS para elementos lineares:

$$
\tau_{K}=\left[4 \frac{\mu}{\rho h_{K}^{2}}+2 \frac{\left\|\mathbf{c}_{h}\right\|_{\infty, K}}{h_{K}}\right]^{-1}, \quad \delta_{K}=4 \mu+2 \rho\left\|\mathbf{c}_{h}\right\|_{\infty, K} h_{K},
$$

onde $h_{K}$ é o tamanho do elemento $K$, e seu valor pode ser computado como o diâmetro da esfera que tem o volume igual a de $K$.

\section{Mini-elemento simplificado}

No mini-elemento tradicional $\left(P_{1}^{+} / P_{1}\right)$, o espaço da velocidade $W_{u, h}$ é o espaço de funções lineares por partes, assim como $Q_{h}$, mas enriquecido com uma função bolha $\phi^{b}$ em cada elemento $K \subset \mathbb{R}^{d}$, onde tal função é um polinômio cúbico (em 2D) ou quártico (em 3D) que se anula em todo bordo do elemento correspondente. Na versão simplificada, os graus de liberdade associados às bolhas são estaticamente eliminados 
de modo aproximado (ou exato, quando possível). A seguir, é descrito como realizar este procedimento.

A velocidade $\mathbf{u}_{h}^{n}$ pode ser decomposta da seguinte forma:

$$
\mathbf{u}_{h}^{n}=\mathbf{u}_{h}^{b, n}+\tilde{\mathbf{u}}_{h}^{n}
$$

onde $\mathbf{u}_{h}^{b, n}$ é a parte da velocidade ponderada com as funções bolhas e $\tilde{\mathbf{u}}_{h}^{n}$ a parte pesada com as funções lineares por partes. Da mesma forma, o resíduo do momento linear associado à função bolha $\phi^{b}$ que tem suporte no elemento $K$ pode ser decomposto nos seguintes resíduos

$$
\begin{aligned}
\mathcal{F}_{u}^{b}\left(\mathbf{u}_{h}^{n+\theta}, \phi^{b}\right) \doteq & \left(\rho \delta_{t} \mathbf{u}_{h}^{b, n}, \phi^{b}\right)+a\left(\mathbf{u}_{h}^{b, n+\theta}, \phi^{b}\right)+c\left(\mathbf{c}_{h} ; \mathbf{u}_{h}^{b, n+\theta}, \phi^{b}\right) \\
\widetilde{\mathcal{F}}_{u}\left(\mathbf{u}_{h}^{n+\theta}, \phi^{b}\right) \doteq & \left(\rho \delta_{t} \tilde{\mathbf{u}}_{h}^{n}, \phi^{b}\right)+a\left(\tilde{\mathbf{u}}_{h}^{n+\theta}, \phi^{b}\right)+c\left(\mathbf{c}_{h} ; \tilde{\mathbf{u}}_{h}^{n+\theta}, \phi^{b}\right)- \\
& -b\left(p_{h}^{n+1}, \phi^{b}\right)-\mathcal{P}\left(\phi^{b}\right),
\end{aligned}
$$

tal que vale a igualdade (com a dependência da pressão omitida para não sobrecarregar a notação)

$$
\mathcal{F}_{u}^{b}\left(\mathbf{u}_{h}^{n+\theta}, \phi^{b}\right)+\widetilde{\mathcal{F}}_{u}\left(\mathbf{u}_{h}^{n+\theta}, \phi^{b}\right)=\mathbf{0} .
$$

Os coeficientes da bolha $\underline{\mathbf{u}}^{b}$ no tempo $n+1$ deste elemento $K$ são dados pela solução do seguinte sistema $d \times d$ :

$$
(\mathbf{M}+\theta \mathbf{A}) \underline{\mathbf{u}}^{b, n+1}=-\widetilde{\mathcal{F}}_{u}\left(\mathbf{u}_{h}^{n+\theta}, \phi^{b}\right)+(\mathbf{M}+(\theta-1) \mathbf{A}) \underline{\mathbf{u}}^{b, n}
$$

onde

$$
\begin{aligned}
\mathbf{M} & \doteq \mathbf{I} \int_{\Omega_{e}^{n+\theta}} \rho \frac{\left(\phi^{b}\right)^{2}}{\Delta t} \\
\mathbf{A} & \doteq \mathbf{I} \int_{\Omega_{e}^{n+\theta}} \rho\left(\mathbf{c}_{h} \cdot \nabla \phi^{b}\right) \phi^{b}+\int_{\Omega_{e}^{n+\theta}} \mu\left(\mathbf{I}\left|\nabla \phi^{b}\right|^{2}+\nabla \phi^{b} \otimes \nabla \phi^{b}\right),
\end{aligned}
$$

e I é a matriz identidade. São feitas as seguintes observações:

- O sistema em (2.59) é um problema não linear, pois $\mathbf{c}_{h}$ depende dos coeficientes das bolhas. Entretanto, é possível substituir $\mathbf{c}_{h}$ por $\tilde{\mathbf{c}}_{h}$ sem perda de precisão considerável, pois os valores numéricos dos coeficientes $\underline{\mathbf{u}}^{b}$ são pequenos, e então termos de $\mathcal{O}\left(\left\|\underline{\mathbf{u}}^{b}\right\|^{2}\right)$ podem ser desprezados. Veja por exemplo os resultados de Soulaimani et al. [45].

- É preciso ter os coeficientes das bolhas no tempo $n$ para computar os coeficientes no tempo $n+1$, o que faz com que seja necessário armazená-los. Entretanto, tais 
coeficientes também podem ser desconsiderados, i.e., $\underline{\mathbf{u}}^{b, n}=\mathbf{0}$, como é justificado pelo resultados numéricos. Dessa forma, a Eq. (2.59) fica

$$
(\mathbf{M}+\theta \mathbf{A}) \underline{\mathbf{u}}^{b, n+1}=-\widetilde{\mathcal{F}}_{u}\left(\phi^{b}\right) .
$$

Isto é equivalente a considerar que o elemento é o $P_{1} / P_{1}$ e os coeficientes das bolhas são apenas um artifício de estabilização, de modo que eles não são utilizados na interpolação da solução final.

- Tais simplificações são puramente heurísticas e somente os resultados numéricos são usados para justificá-las.

\section{Elemento $P_{2} / P_{1}$}

Este tradicional elemento de Taylor-Hood satisfaz a condição inf-sup e portanto não é preciso alterações na formulação variacional. A velocidade é interpolada por polinômios quadráticos por partes e a pressão por polinômios lineares por partes. Este é o elemento quadrático mais simples em termos de implementação, onde a única complicação adicional sobre os elementos lineares apresentados é a necessidade de uma estrutura de dados para gerenciar os graus de liberdade associados às arestas.

\section{Elemento $P_{2}^{+} / P_{1}^{K}$ simplificado}

O elemento $P_{2}^{+} / P_{1}^{K}$, pertencente a família de elementos Crouzeix-Raviart, satisfaz a condição inf-sup e portanto não é preciso de alterações na formulação variacional. Neste elemento, a velocidade é interpolada por polinômios quadráticos estendidos e a pressão por polinômios lineares descontínuos entre os elementos. Em 3D, o espaço da velocidade é enriquecido com funções bolhas não apenas nos centróides dos elementos, mas também nas interfaces entre eles (cf. Crouzeix e Raviart [15]).

A versão simplificada consiste em eliminar estaticamente não só os graus de liberdade associados às bolhas mas também os associados ao gradiente de pressão. Estes procedimentos, os quais são descritos adiante, também podem ser encontrados em $\mathrm{Cu}$ velier et al. [16] para o caso estacionário em 2D. Em 3D, este elemento não é considerado neste trabalho.

Antes de realizar a simplificação, é escolhido uma base diferente da tradicional para o espaço da pressão $P_{1}$ em cada elemento (não é necessário, mas simplifica os cálculos). Seja $K \subset \mathbb{R}^{d}$ um elemento, $\hat{K}$ o elemento de referência e $\mathcal{N}=\left\{N^{i}\right\}_{i=1}^{d+1}$ a base do 
espaço dual de $P_{1}(K)^{*}$. Em vez de escolher os tradicionais funcionais lineares $N^{i}$ como sendo

$$
N^{i}(f)=f\left(\mathbf{x}^{i}\right), \quad f \in P_{1}(K),
$$

onde $\mathbf{x}^{i}$ são as coordenadas dos nós de $K$, os quais levam à base tradicional $\left\{\psi^{j}\right\}_{j=1}^{d+1}$ dada por (para elementos simpliciais)

$$
\left\{x_{1}, \ldots, x_{d}, 1-x_{1} \ldots-x_{d}\right\}, \quad \boldsymbol{x} \in \hat{K},
$$

é escolhido

$$
N^{i}(f)=\left.\frac{\partial f}{\partial x_{i}}\right|_{\mathbf{x}^{(c)}}, \quad N^{d+1}(f)=\frac{1}{|K|} \int_{K} f,
$$

onde $|K|$ é o volume do elemento e $\mathbf{x}^{(c)}$ é o baricentro de $K$ definido por

$$
\mathbf{x}^{(c)}=\frac{1}{|K|} \int_{K} \boldsymbol{x}
$$

de forma que a nova base $\left\{\psi^{j}\right\}_{j=1}^{d+1}$ dual à $\mathcal{N}$ (i.e., $N^{i}\left(\psi^{j}\right)=\delta_{i j}$ ) seja dada por

$$
\left\{1, x_{1}-x_{1}^{(c)}, \ldots, x_{d}-x_{d}^{(c)}\right\}, \quad \boldsymbol{x} \in K .
$$

Dessa forma, a pressão no elemento $K$ é escrita como

$$
p_{h}(\boldsymbol{x})=\bar{p}+\left.\nabla p\right|_{\boldsymbol{x}^{(c)}} \cdot\left(\boldsymbol{x}-\boldsymbol{x}^{(c)}\right), \quad \boldsymbol{x} \in K,
$$

onde $\bar{p}$ (a pressão média em $K$ ) e $\left.\nabla p\right|_{\boldsymbol{x}^{(c)}}$ (o gradiente da pressão avaliada no baricentro de $K$ ) são os graus de liberdade. O espaço da pressão escrito na nova base será denotado por $P_{1}^{K}$ e o escrito na base tradicional por $P_{1}^{\widehat{K}}$.

Para eliminar as bolhas no elemento $K$, é usado a restrição de incompressibilidade

$$
b\left(\psi^{i}, \nabla \cdot \mathbf{u}_{h}^{n+\theta}\right)=b\left(\psi^{i}, \nabla \cdot \mathbf{u}_{h}^{b, n+\theta}\right)+b\left(\psi^{i}, \nabla \cdot \tilde{\mathbf{u}}_{h}^{n+\theta}\right)=0, \quad \boldsymbol{x} \in K,
$$

que pode ser escrita como

$$
b\left(\psi^{i}, \nabla \phi^{b}\right) \cdot \underline{\mathbf{u}}^{b, n+\theta}=-b\left(\psi^{i}, \nabla \cdot \tilde{\mathbf{u}}_{h}^{n+\theta}\right), \quad \boldsymbol{x} \in K, .
$$

O lado direiro da equação anterior pode ser computado no elemento $K$ graças à compacidade de $\psi^{i}$. Adotando os valores $1, \ldots, d$ para o índice $i$, então as $d$ Eqs. (2.69)

*Ver detalhes sobre espaço dual, construção de espaços de elementos finitos, etc., em Brenner e Scott [6]. 
formam o sistema

$$
\int_{K}\left(\boldsymbol{x}-\boldsymbol{x}^{(c)}\right) \otimes \nabla \phi^{b} \cdot \underline{\mathbf{u}}^{b, n+\theta}=-\int_{K}\left(\boldsymbol{x}-\boldsymbol{x}^{(c)}\right) \nabla \cdot \tilde{\mathbf{u}}_{h}^{n+\theta} .
$$

Integrando por partes a matriz e usando o fato que $\phi^{b}$ anula-se no bordo de $K$, obtém-se

$$
\underline{\mathbf{u}}^{b, n+\theta}=\left[\int_{K} \phi^{b}\right]^{-1} \int_{K}\left(\boldsymbol{x}-\boldsymbol{x}^{(c)}\right) \nabla \cdot \tilde{\mathbf{u}}_{h}^{n+\theta} .
$$

Para eliminar o gradiente de pressão, o procedimento é análogo. Considere o termo da pressão na quantidade de movimento linear, i.e., $b\left(p_{h}, \nabla \cdot \mathbf{w}_{h}\right)$. Escolhendo-se $\mathbf{w}_{h}=$ $\phi^{b} \mathbf{e}^{m}, m=1, . ., d$, onde $\mathbf{e}^{m}$ são os versores da base canônica e $\phi^{b}$ é a bolha de $K$, vem

$$
\begin{aligned}
\int_{\Omega} p_{h} \nabla \phi^{b} & =\int_{K}\left(\bar{p}+\left.\nabla p\right|_{\boldsymbol{x}^{(c)}} \cdot\left(\boldsymbol{x}-\boldsymbol{x}^{(c)}\right)\right) \nabla \phi^{b} \\
& =\int_{K} \bar{p} \nabla \phi^{b}+\left.\int_{K}\left[\nabla \phi^{b} \otimes\left(\boldsymbol{x}-\boldsymbol{x}^{(c)}\right)\right] \cdot \nabla p\right|_{\boldsymbol{x}^{(c)}} \\
& =\int_{K} \bar{p} \nabla \phi^{b}-\left.\left[\int_{K} \phi^{b}\right]^{-1} \nabla p\right|_{\boldsymbol{x}^{(c)}}
\end{aligned}
$$

Supondo que o resíduo da quantidade da movimento sem o termo de pressão e ponderado com as funções $\mathbf{w}_{h}=\phi^{b} \mathbf{e}^{m}$ seja escrito na forma $\mathcal{F}_{u}^{(u)}\left(\mathbf{u}_{h}^{n+\theta}, \phi^{b}\right)$, então

$$
\begin{aligned}
0 & =\mathcal{F}_{u}^{(u)}\left(\mathbf{u}_{h}^{n+\theta}, \phi^{b}\right)-\int_{\Omega} p_{h} \nabla \phi^{b} \\
& =\mathcal{F}_{u}^{(u)}\left(\mathbf{u}_{h}^{n+\theta}, \phi^{b}\right)-\int_{K} \bar{p} \nabla \phi^{b}-\left.\left[\int_{K} \phi^{b}\right]^{-1} \nabla p\right|_{\boldsymbol{x}^{(c)}},
\end{aligned}
$$

e portanto, em cada elemento $K$,

$$
\left.\nabla p\right|_{\boldsymbol{x}^{(c)}}=\left(\int_{K} \phi^{b}\right)\left(\mathcal{F}_{u}^{(u)}\left(\mathbf{u}_{h}^{n+\theta}, \phi^{b}\right)-\int_{K} \bar{p} \nabla \phi^{b}\right) .
$$

Seguem algumas observações:

- Pelo mesmo motivo do mini-elemento, os coeficientes $\underline{\mathbf{u}}^{b, n}$ são reinicializados a zero no início de cada passo de tempo.

- Ao final das contas, a estrutura do jacobiano e do resíduo do método de Newton (i.e., $J^{k} \Delta x^{k}=-\mathcal{F}^{k}$ ) para este elemento, é equivalente ao do elemento $P_{2}-P_{0}$, com modificações apenas na matriz no bloco velocidade-velocidade e no resíduo da quantidade de movimento, ou seja

$$
\left[\begin{array}{cc}
A+\text { modificações } & G \\
D & 0
\end{array}\right]\left[\begin{array}{c}
\Delta u \\
\Delta p
\end{array}\right]^{k}=-\left[\begin{array}{c}
\mathcal{F}_{u}+\operatorname{modificações~}\left(\mathbf{u}_{h}\right) \\
\mathcal{F}_{p}
\end{array}\right]
$$




\subsection{A condição de contorno de não penetração}

A condição de contorno de Neumann aparece naturalmente na formulação variacional na forma de uma integral, enquanto que a condição de Dirichlet é imposta fortemente substituindo-se os $d$ resíduos do momento linear $\mathcal{F}_{u}\left(\mathbf{u}_{h}, p_{h}, \phi^{i}\right)$, correspondentes ao nó $i$, pelos $d$ resíduos

$$
\mathbf{u}^{i}(t)-\mathbf{u}_{\Gamma}\left(\mathbf{x}^{i}, t\right)=0, \quad \mathbf{x}^{(i)} \in \Gamma_{D},
$$

em cada nó $i$ do contorno, onde $\mathbf{u}_{\Gamma}$ é a velocidade prescrita introduzida em (2.11a). Quando apenas a componente normal da velocidade é prescrita (chamado aqui de condição de não penetração, ver Eq. (2.11d)), apenas as equações que correspondem à normal de $\mathcal{F}_{u}\left(\mathbf{u}_{h}, p_{h}, \phi^{i}\right)$ são substituídas pela componente normal de (2.76), o que é um procedimento trivial quando a normal está alinhada com algum dos eixos $\mathbf{e}^{m}$. Quando não está alinhada, já não é tão claro como substituir $\mathcal{F}_{u}\left(\mathbf{u}_{h}, p_{h}, \phi^{i}\right)$ por $(2.76)$, mas existem várias alternativas para tratar esta situação e algumas delas são discutidas a seguir (ver também Ainsworth [1]).

\subsubsection{Rotações/Projeções}

Em Engelman et al. [21] (ver também Gresho e Sani [27]) é proposto um método que consiste em rotacionar as equações de momento linear dos nós onde são impostas a condição de não penetração em superfícies sólidas de modo a alinhar a normal n a algum eixo $\mathbf{e}^{m}$. Neste trabalho, são propostas algumas pequenas modificações neste método, onde em vez de usar rotações, são usadas projeções. Por uma questão de completeza, o método de rotações é apresentado.

Em cada nó $i$, pode ser definida a seguinte matriz de rotação $\mathbf{R}^{i} \in \mathbb{R}^{d \times d}$

$$
\mathbf{R}^{i} \doteq\left\{\left[\begin{array}{c}
\left(\mathbf{n}^{i}\right)^{T} \\
\left(\boldsymbol{\tau}_{a}^{i}\right)^{T} \\
\left(\boldsymbol{\tau}_{b}^{i}\right)^{T}
\end{array}\right], \text { se } \mathbf{x}^{i} \in \Gamma_{M} ; \quad \mathbf{I}_{d}, \text { caso contrário }\right\},
$$

onde $\mathbf{I}_{d}$ é a matriz identidade, $\mathbf{n}^{i}$ a normal em $\mathbf{x}^{i}$ e $\boldsymbol{\tau}_{a}^{i}$ e $\boldsymbol{\tau}_{b}^{i}$ são dois versores tangenciais em $\mathbf{x}^{i}$ e ortogonais entre si (obviamente em 2D, só uma tangencial é definida). A normal é um dado do problema, uma vez que a superfície sólida é conhecida (neste trabalho, esta é representada por uma função implícita), enquanto as tangenciais devem ser definidas. A velocidade rotacionada está relacionada com a velocidade $\mathbf{u}^{i}=\left(u_{1}^{i}, u_{2}^{i}, u_{3}^{i}\right)$ 
da seguinte forma

$$
\mathbf{u}_{R}^{i}=\left[u_{n}^{i}, u_{\tau a}^{i}, u_{\tau b}^{i}\right]^{T}=\mathbf{R}^{i} \cdot \mathbf{u}^{i}
$$

Deve-se observar que $\mathbf{R}^{i}$ é uma matriz ortogonal, portanto $\mathbf{R}^{i} \cdot\left(\mathbf{R}^{i}\right)^{T}=\mathbf{I}_{d}$. Sendo $\mathbf{R}$ a matriz de rotação global, i.e., a composição das matrizes $\mathbf{R}^{i}$, dada por

$$
\mathbf{R} \doteq\left[\begin{array}{ccc}
\mathbf{R}^{1} & & \\
& \ddots & \\
& & \mathbf{R}^{\eta}
\end{array}\right]
$$

o sistema de equações do momento linear do problema pode ser escrito na forma

$$
\mathbf{A} \underline{\mathbf{u}}+\mathbf{G} \underline{p}=\mathbf{f} .
$$

onde $\underline{\mathbf{u}}$ é o vetor de coeficientes (assim como $p$ ) ordenado de acordo com $\mathbf{R}$. Multiplicando (2.80) por $\mathbf{R}$ e substituindo $\underline{\mathbf{u}}$ por $\mathbf{R}^{T} \underline{\mathbf{u}_{R}}$, onde $\underline{\mathbf{u}_{R}}$ é a composição de $\mathbf{u}_{R}^{i}$, obtém-se o método proposto por Engelman et al. [21]:

$$
\mathbf{R A R}^{T} \underline{\mathbf{u}_{R}}+\mathbf{R G} \underline{p}=\mathbf{R f} .
$$

Com o sistema escrito na forma anterior, é possível efetuar o procedimento usual de impor as condições de contorno. As rotações de $\mathbf{A}$ podem ser montadas a nível de elemento, i.e., $\mathbf{R} \mathbf{A} \mathbf{R}^{T}$ é a montagem das matrizes $\mathbf{R}^{(e)} \mathbf{A}^{(e)} \mathbf{R}^{(e), T}$ de todos os elementos $e$, onde, mais uma vez, cada $\mathbf{R}^{(e)}$ é a composição de $\mathbf{R}^{i}$ s, para todo nó $i$ contido em $e$.

Pequenas modificações neste método são propostas a seguir. Seja $\hat{\mathbf{I}}_{R}^{i}$ a matriz identidade correspondente ao nó $i$, tal que o elemento diagonal correspondente à linha da normal é nulo se o nó está em $\Gamma_{M}$, isto é,

$$
\hat{\mathbf{I}}_{R}^{i} \doteq\left\{\left[\begin{array}{ccc}
0 & 0 & 0 \\
0 & 1 & 0 \\
0 & 0 & 1
\end{array}\right], \text { se } \mathbf{x}^{i} \in \Gamma_{M} ; \quad \mathbf{I}_{d}, \text { caso contrário }\right\},
$$

e seja $\hat{\mathbf{I}}_{R}$ a matriz global de $\hat{\mathbf{I}}_{R}^{i}$. As condições de não penetração são impostas em (2.81) substituindo-se as equações (linhas) correspondentes às componentes normais pelos valores da velocidade normal, o que é equivalente a multiplicar (2.81) por $\hat{\mathbf{I}}_{R} \mathrm{e}$ somar $\left(\mathbf{I}-\hat{\mathbf{I}}_{R}\right) \underline{\mathbf{u}}_{R}$ e $\left(\mathbf{I}-\hat{\mathbf{I}}_{R}\right) \underline{\mathbf{U}}_{R}$ em seu lado esquerdo e direito, respectivamente, ou seja,

$$
\left(\mathbf{I}-\hat{\mathbf{I}}_{R}+\hat{\mathbf{I}}_{R} \mathbf{R A R} \mathbf{R}^{T}\right) \underline{\mathbf{u}}_{R}+\hat{\mathbf{I}}_{R} \mathbf{R G} \underline{p}=\hat{\mathbf{I}}_{R} \mathbf{R f}+\left(\mathbf{I}-\hat{\mathbf{I}}_{R}\right) \underline{\mathbf{U}}_{R},
$$

onde $\underline{\mathbf{U}}_{R}$ é o vetor com os valores exatos da velocidade rotacionada de $\mathbf{u}$, tal que em 
cada nó $i$ do contorno:

$$
\underline{\mathbf{U}}_{R}^{i}=\left[U_{n}\left(\mathbf{x}^{i}\right), 0,0\right]^{T} .
$$

Multiplicando (2.83) por $\mathbf{R}^{T}$ e substituindo $\underline{\mathbf{u}}_{R}$ por $\mathbf{R} \underline{\mathbf{u}}$, obtém-se o método proposto

$$
(\mathbf{I}-\mathbf{P}+\mathbf{P A}) \underline{\mathbf{u}}+\mathbf{P G} \underline{p}=\mathbf{P f}+(\mathbf{I}-\mathbf{P}) \underline{\mathbf{U}},
$$

onde $\underline{\mathbf{U}}=\mathbf{R}^{T} \underline{\mathbf{U}}_{R}, \mathbf{P}$ é a matriz de projeção dada pela composição das matrizes $\mathbf{P}^{i}$, e

$$
\mathbf{P}^{i} \doteq\left\{\mathbf{I}_{d}-\mathbf{n}^{i} \otimes \mathbf{n}^{i}, \text { se } \mathbf{x}^{i} \in \Gamma_{M} ; \quad \mathbf{I}_{d}, \text { caso contrário }\right\} .
$$

Em termos de resíduos, o sistema (2.85) pode ser apresentado de forma mais simples como

$$
\mathbf{P}^{i} \cdot \mathcal{F}_{u}\left(\mathbf{u}_{h}, p_{h}, \phi^{i}\right)+\left(\mathbf{I}_{d}-\mathbf{P}^{i}\right)\left(\mathbf{u}^{i}-U_{n}\left(\mathbf{x}^{i}\right) \mathbf{n}^{i}\right)=\mathbf{0}, \quad i \in \eta .
$$

onde lembrando que $\eta$ é o conjunto de nós da velocidade. Seguem algumas observações sobre este método.

- Assim como no método por rotações em (2.81), no método por projeções (2.85) também é possível construir as matrizes em nível de elemento.

- Em relação à implementação, uma vantagem sobre o método por rotações é que não é necessário se trabalhar com os coeficientes rotacionados $\underline{\mathbf{u}_{R}}$, os quais devem ser rotacionados de volta antes de se plotar resultados, computar velocidade da malha, etc..

- Outra vantagem é que não é necessário definir os versores tangencias $\tau_{a}$ e $\tau_{b}$, para os quais em 3D existem infinitas possiblidades de escolha. Dessa forma, no método por projeções, o mesmo código 2D pode ser aproveitado para 3D.

- Os dois métodos são obviamente equivalentes, porém o método proposto é mais conveniente do ponto de vista prático, pelas razões discutidas.

\subsubsection{Imposição fraca da condição de Dirichlet}

Esta é uma categoria de métodos usada mais em outros contextos, por exemplo, em conjunto com métodos de fronteira imersa, e por isso será apenas brevemente discutida. Os principais métodos são apresentados a seguir, e suas variantes podem ser encontradas nas referências citadas. 


\section{Multiplicadores de Lagrange}

Neste método, a tensãonormal é introduzida no problema como um multiplicador de lagrange $\lambda \doteq \mathbf{n} \cdot \boldsymbol{\sigma} \cdot \mathbf{n}$, e então é acrescentado no resíduo $\mathcal{F}_{u}\left(\mathbf{u}_{h}, p_{h}, \phi^{i}\right)$ o termo

$$
\int_{\Gamma_{M}} \lambda \phi^{i} \mathbf{n}
$$

equanto a condição de não penetração é imposta de forma fraca:

$$
\int_{\Gamma_{M}} \zeta^{i}\left(\mathbf{u}_{h} \cdot \mathbf{n}-U_{n}\right)=0
$$

onde $\zeta^{i}$ são as funções de interpolação de $\lambda$. O problema deste método é que são introduzidas novas incógnitas no problema, além de o espaço da função $\lambda$ ter que satisfazer um condição inf-sup, não podendo o mesmo ser arbitrário (ver Stenberg $[48])$.

\section{Penalização}

Neste método, no lugar da condição de não penetração $\mathbf{u}_{h} \cdot \mathbf{n}=U_{n}$, é considerada a condição de Robin

$$
\varepsilon \mathbf{n} \cdot \boldsymbol{\sigma} \cdot \mathbf{n}+\mathbf{u}_{h} \cdot \mathbf{n}=U_{n},
$$

onde $\varepsilon$ é um parâmetro ao qual deve ser atribuído um valor pequeno. Com isso, o termo $\left(\mathbf{u}_{h} \cdot \mathbf{n}-U_{n}\right) / \varepsilon$ aparece na intergal em $\Gamma_{M}$ no lugar de $\lambda$ em (2.88). O problema deste método é que o valor pequeno de $\varepsilon$ leva a uma má condição na matriz de elementos finitos, além da escolha do valor do parâmetro em si.

\section{Método de Nitsche.}

Tal método é uma versão aprimorada do da penalização. Assim como sua antecessora, ela também necessita de uma parâmetro ajustável, o qual não é fácil de ser determinado para as equações de Navier-Stokes. Descrições deste método podem ser encontrados em Hautefeuille et al. [30].

\section{Campo de tensor de tensões.}

Em Gerstenberger e Wall [26] é proposto um método (ver também em Baltussen et al. [2]) semelhante ao do primeiro item, onde em vez de a tensão normal $\mathbf{n} \cdot \boldsymbol{\sigma} \cdot \mathbf{n}$ na superfície ser adicionada como incógnita, um campo de tensor de tensões $\hat{\boldsymbol{\sigma}}$ é adicionado em todo o domínio. O truque deste método é a possibilidade de se escolher um espaço descontíno 
para $\hat{\boldsymbol{\sigma}}$ de modo que seus graus de liberdade possam ser estaticamente eliminados, não introduzindo portanto, efetivamente, nenhuma incógnita ao problema. Além disso, não é necessário nenhum parâmetro ajustável neste método. Contudo, surgem integrais no contorno $\Gamma_{M}$ do tipo

$$
\int_{\Gamma_{M}} \mathbf{w}_{h} \cdot \nabla \mathbf{u}_{h} \cdot \mathbf{n}
$$

que dependem dos coeficientes de $\mathbf{u}_{h}$ no interior do domínio (por causa de $\nabla \mathbf{u}_{h}$ ), o que pode ser inconveniente, em termos de implementação, para computar tal intergral, dependendo da biblioteca de elementos finitos utilizada.

\subsection{Movimentação da malha}

Alguns poucos elementos distorcidos da malha podem prejudicar tanto a precisão do método quanto o condicionamento da matriz (um estudo da influência da qualidade de elementos simpliciais lineares pode ser encontrado em Shewchuk [42]). Como os casos testados neste trabalho (essencialmente gotas que se espalham em superfícies planas) há apenas distorções moderadas da malha, um modelo simples de movimentação de pontos será adotado, deixando métodos com operações topológicas como futuros objetos de estudo.

Em razão de malhas com elementos curvos (quadráticos, especificamente) também serem consideradas, uma distinção é feita entre seus graus de liberdade $\mathbf{x}^{k}$; os que estão no encontro das arestas são chamados de vértices enquanto que os associados às arestas são chamados de nós da aresta, ou nós de alta ordem. Quando dito apenas nós, estará se referindo tanto aos vértices como aos nós de alta ordem.

\subsubsection{Suavização Laplaciana restrita}

Os métodos de melhoria da malha podem ser classificados em duas categorias: suavização, que consiste em reposicionar os vértices da malha, e transformação topológica, que consiste em substituir um conjunto de elementos por outro. Obviamente, ambas são feitas de modo a aumentar a qualidade da malha. Nesta última categoria, ocorrem mudanças na conectividade da malha e consequentemente na estrutura esparsa da matriz de elementos finitos, o que pode consumir muito tempo de CPU. Por isso, e por questão de simplicidade, a primeira estratégia é adotada.

Um método muito popular de suavização é a suavização Laplaciana, que consiste simplesmente em mover cada vértice para a média das coordenadas dos vértices adja- 

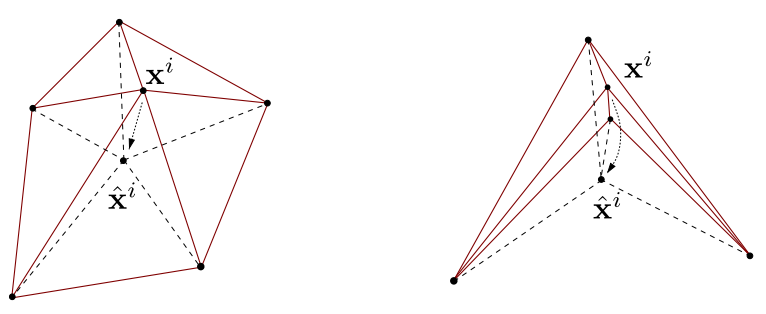

Figura 2.2: Exemplo de reposicionamento do vértice via suavização Laplaciana: caso convexo e não convexo (figura retirada de Sousa [46]). O vetor $\hat{\mathbf{x}}^{i}$ é a nova posição do vértice.

centes, ou seja

$$
\mathbf{x}^{i} \leftarrow \frac{1}{n^{i}} \sum_{j \in S(i)} \mathbf{x}^{j},
$$

onde $S(i)$ é o conjunto de vértices adjacentes ao vértice $i$ e $n^{i}$ é o número de vértices em $S(i)$. No caso de malhas de elementos curvos, cada nó de alta ordem que não esteja no bordo é movido para a média das coordenadas das extremidades da aresta $A(i)$ e $B(i)$, i.e.,

$$
\mathbf{x}^{i} \leftarrow \frac{1}{2}\left(\mathbf{x}^{A(i)}+\mathbf{x}^{B(i)}\right)
$$

mas depois que todos os vértices da malha já tiverem sido suavizados, de forma que ao final do procedimento todas as arestas no interior do domínio fiquem retas. Os nós que estão no bordo são descritos mais adiante.

O incoveniente deste método é que quando $S(i)$ forma um polítopo ${ }^{\dagger}$ não convexo pode surgir um elemento inválido, como ilustra a Fig. (2.2). Por isso, uma versão modificada conhecida na literatura como suavização Laplaciana restrita $a^{\ddagger}$ é adotada. Nesta variante, a qualidade do conjunto de elementos incidentes ao vértice é medida antes e depois do reposicionamento deste. Se a qualidade depois do reposicionamento é pior, então a coordenada original do vértice é restaurada. Os nós de alta ordem são tratados da mesma forma que no método original.

\subsubsection{Medida de qualidade}

Existem vários tipos de medida de qualidade de um elemento. O que será adotado é o comprimento de volume, pois este é fácil de ser implementado e rápido de ser computado em relação aos outros tipos. Sua característica é selecionar os elementos

\footnotetext{
${ }^{\dagger}$ Generalização dos conceitos de polígono e poliedro.

${ }_{\ddagger}^{\ddagger} \mathrm{Na}$ língua inglesa é encontrada tanto como smart Laplacian smooth como constrained Laplacian smooth.
} 

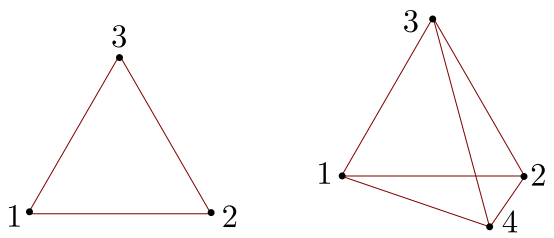

Figura 2.3: Orientação dos vértices adotada para as fórmulas de qualidade.

"gordos" (Klingner [32]). A qualidade de um elemento é dada por (cf. Shewchuk [42])

$$
\text { triângulo: } \frac{4}{\sqrt{3}} \frac{A}{\ell_{r m s}^{2}}, \quad \text { tetraedro: } 6 \sqrt{2} \frac{V}{\ell_{r m s}^{3}},
$$

onde

$$
\ell_{r m s}=\sqrt{\frac{1}{e} \sum_{i=1}^{e} \ell_{i}^{2}}
$$

é o comprimento quadrático médio das arestas do elemento, $A$ é a área com sinal e $V$ é o volume com sinal do elemento. As constantes multiplicativas são para normalizar a medida de modo que a melhor qualidade (i.e., o valor máximo) valha 1 . A área e o volume dados por

$$
A=\frac{1}{2} \operatorname{det}\left[\begin{array}{rrr}
1 & x_{1}^{1} & x_{2}^{1} \\
1 & x_{1}^{2} & x_{2}^{2} \\
1 & x_{1}^{3} & x_{2}^{3}
\end{array}\right], \quad V=\frac{1}{6} \operatorname{det}\left[\begin{array}{rrrr}
1 & x_{1}^{1} & x_{2}^{1} & x_{3}^{1} \\
1 & x_{1}^{2} & x_{2}^{2} & x_{3}^{2} \\
1 & x_{1}^{3} & x_{2}^{3} & x_{3}^{3} \\
1 & x_{1}^{4} & x_{2}^{4} & x_{3}^{4}
\end{array}\right],
$$

onde $\left(x_{1}^{i}, . ., x_{d}^{i}\right)$ é a coordenada do vértice $i$, têm sinal negativo quando a orientação dos vértices é diferente da ilustrada na Fig. (2.3). A qualidade do conjunto de elementos incidentes ao vértice $i$, denotado por $\mathcal{Q}(i)$, é definida como a qualidade do pior elemento desse conjunto.

Na medida de qualidade de elementos curvos, os nós de alta ordem são ignorados por questão de simplicidade. Vale observar que referências em controle de qualidade em malhas dinâmicas para estes tipos de elementos são escassas na literatura. Em 2D, um estudo é feito em Cardoze et al. [11] para elementos triangulares.

\subsubsection{Suavização no bordo e normais}

Para finalizar, uma observação sobre suavização de nós no bordo da malha é feita. $\mathrm{Na}$ equação (2.43) foi visto que, no bordo, os nós têm liberdade de se moverem arbitrariamente no plano tangente, e em alguns casos é necessário utilizar essa liberdade 
para mantê-los equidistribuídos no bordo de modo a evitar o surgimento de elementos inválidos. O problema é que não é definida uma normal nos vértices para que se possa determinar o plano tangente. Um método bastante comum de se calcular a normal, consistente com as restrições de incompressibilidade, é proposto em Engelman et al. [21], onde em qualquer nó $i$, a normal é dada por

$$
\mathbf{n}^{i}=\frac{\int_{\Omega} \nabla \vartheta^{i}}{\left\|\int_{\Omega} \nabla \vartheta^{i}\right\|}=\frac{\int_{\Gamma} \mathbf{n} \vartheta^{i}}{\left\|\int_{\Gamma} \mathbf{n} \vartheta^{i}\right\|}
$$

Apesar de funcionar bem em 2D para elementos lineares e curvos e em 3D para elementos lineares, não funciona em 3D para elementos curvos, pois nos vértices tem-se que

$$
\int_{\Gamma} \vartheta^{i}=0
$$

ou seja, nos vértices em um plano onde $\mathbf{n}$ é constante obtém-se $\mathbf{n}^{i}=\mathbf{0}$, o que obviamente não é desejável. Para o texto que segue, as normais encontradas com este método serão chamadas de normais consistentes. Em razão deste problema com elementos curvos em 3D, outra alternativa será adotada. Em Max [35], é proposta uma alternativa onde as normais encontradas em uma malha superficial de polígonos são exatas quando os nós estão sobre uma casca esférica, independente das formas dos polígonos. Ainda nesta referência, foram feitos um milhão de testes com superfícies aleatórias descritas por polinômios cúbicos, e a normal proposta foi a que apresentou os melhores resultados com respeito ao desvio do ângulo da normal computada com a normal exata.

Em 3D com elementos lineares, para computar a normal $\mathbf{n}^{i}$ no nó $i$, segundo o método de Max [35], pondera-se as normais $\mathbf{n}^{(f)}$ dos triângulos $f$ (vale para polígonos em geral) que contém o nó $i$, ou seja,

$$
\mathbf{n}^{i}=\frac{\sum_{f \in T(i)} \mathbf{n}^{(f)} \omega_{f}}{\left\|\sum_{f \in T(i)} \mathbf{n}^{(f)} \omega_{f}\right\|},
$$

onde $T(i)$ é o conjunto dos triângulos que contém o nó $i$, com os pesos $\omega_{f}$ dados por

$$
\omega_{f}=\frac{\sin \alpha_{f}}{a \cdot b},
$$

onde $\alpha_{f}$ é o ângulo formado entre as arestas do triângulo $f$ que tocam o nó $i$, e $a$ e $b$ são os comprimentos de tais arestas. A alternativa para lidar com os elementos quadráticos adotada aqui, foi dividir cada triângulo em 4 partes conectando os nós das arestas, e então aplicar a fórmula (2.96) em cada nó do subtriângulo.

O conceito da fórmula de Max foi estendida para 2D neste trabalho, ou seja, 


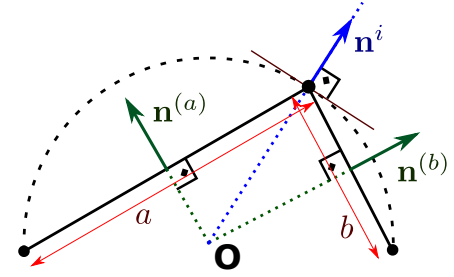

(a)

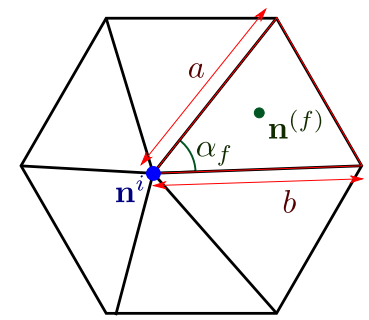

(b)

Figura 2.4: Ilustração das variáveis necessárias para se calcular a normal $\mathbf{n}^{i}$ no vértice $i$, usando a fórmula proposta em Max [35].

procurou-se determinar os pesos $\omega_{f}$ tal que as normais obtidas de uma superfície com os nós sobre uma circunferência sejam exatas. Não é difícil provar que, em 2D,

$$
\mathbf{n}^{i}=\frac{\mathbf{n}^{(a)} / a+\mathbf{n}^{(b)} / b}{\left\|\mathbf{n}^{(a)} / a+\mathbf{n}^{(b)} / b\right\|} .
$$

Para o caso de elementos quadráticos, novamente, as arestas foram divididas em outras duas, e depois aplicado a fórmula anterior em cada subaresta.

Uma vez calculada a normal $\mathbf{n}^{i}$ no vértice $i$, a coordenada $\mathbf{x}^{i}$ é movida tangencialmente da seguinte forma

$$
\mathbf{x}^{i} \leftarrow \mathbf{x}^{i}+\mathbf{P}^{i} \cdot\left(\frac{1}{n^{i}+1} \sum_{j \in T(i)} \mathbf{x}^{j}-\mathbf{x}^{i}\right)
$$

onde $T(i)$ é a união do próprio vértice $i$ com o conjunto de vértices adjacentes ao $i$ que estão no bordo, e $\mathbf{P}^{i}$ a matriz de projeção dada por

$$
\mathbf{P}^{i}=\left(\mathbf{I}-\mathbf{n}^{i} \otimes \mathbf{n}^{i}\right)
$$

sendo I a matrix identidade. De forma análoga para os nós de alta ordem,

$$
\mathrm{x}^{i} \leftarrow \mathrm{x}^{i}+\mathbf{P}^{i} \cdot\left(\frac{1}{3}\left(\mathrm{x}^{A(i)}+\mathrm{x}^{B(i)}\right)-\frac{2}{3} \mathrm{x}^{i}\right)
$$

\subsubsection{Observações}

Os procedimentos feitos na suavização da malha são resumidamente mostrados no Alg. (2.2).

Seguem alguma observações sobre o algoritmo:

- O número de iterações $N$ pode ser definido ou as iterações podem ser feitas até 


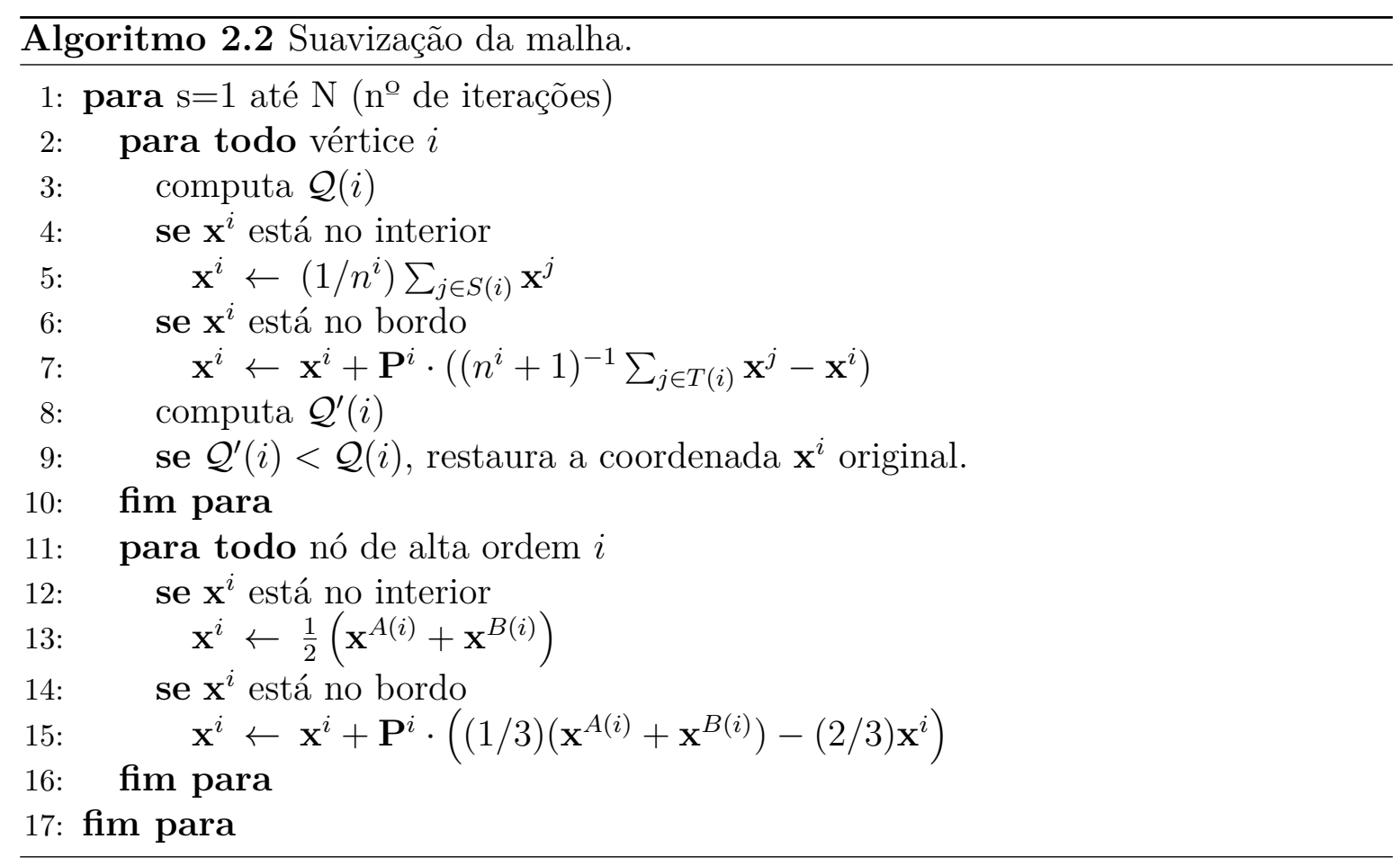

convergir, e.g.,

$$
\left\|\mathbf{x}_{\text {anterior }}^{i}-\mathrm{x}^{i}\right\|<\varepsilon \text {. }
$$

Nos testes numéricos mais simples, escolher $N=2$ mostrou-se suficiente para evitar que ocorram elementos inválidos.

- Na suavização de cada nó $i$ do bordo, a própria coordenada $\mathbf{x}^{i}$ é contabilizada no cálculo das médias, com peso 1 . Isso faz com que o deslocamento no reposicionamento de cada nó $i$ seja menor e consequentemente que a forma da malha seja mais preservada, ao preço de que é preciso mais iterações para os nós ficarem equidistribuídos. Existem métodos mais sofisticados para equidistribuição de pontos na superfície, por exemplo, o descrito em Bonito e Nochetto [3].

- O nós característicos (nós em regiões onde a normal varia abrubtamente excedendo um valor limiar, vide Fig. 2.5) não são suavizados a fim de manter a característica da malha. Em 3D, quando esses nós formam uma linha, é possível suavizá-los com iterações na forma

$$
\mathbf{x}^{i} \leftarrow \mathbf{x}^{i}+\boldsymbol{\tau}^{i}\left(\boldsymbol{\tau}^{i} \cdot \Delta \mathbf{x}^{i}\right)
$$

onde $\Delta \mathbf{x}^{i}$ é um deslocamento calculado por uma média e $\boldsymbol{\tau}^{i}$ um versor tangente à linha. 

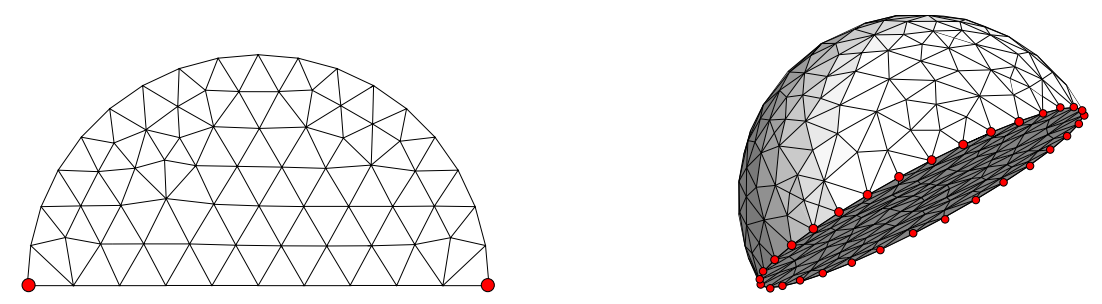

Figura 2.5: Nós característicos (realçados em vermelho). 


\section{Capítulo 3}

\section{Exemplos e testes numéricos para a formulação ALE}

Neste capítulo, são apresentadas validações do código de elementos finitos elaborado que implementa os métodos de discretização espaciais e temporais descritos no primeiro capítulo, a medida que são feitas discussões gerais sobre precisão e desempenho. Em todos os casos tratados, os sistemas de equações não lineares resultantes são resolvidos com o método de Newton, onde os sistemas lineares internos são resolvidos utilizando-se o GMRES (com tolerância de $10^{-12}$ ) com o precondicionador ASM (Additive Schwarz Method), sendo cada bloco precondicionado com ILU(0) (LU incompleto com nível 0 de preenchimento).

Em alguns casos, são considerados problemas com soluções manufaturadas. Em problemas deste tipo, a velocidade $\mathbf{u}$ e a pressão $p$ são escolhidas (para $\mathbf{u}$, obviamente é escolhido um campo solenoidal), e então a força de campo $\boldsymbol{g}$ nas equações de NavierStokes (2.10a) é ajustada para que a escolha $(\mathbf{u}, p)$ seja solução de tais equações.

Para o texto que segue, são empregadas as seguintes siglas para os elementos: 
Tabela 3.1: Siglas dos elementos.

\begin{tabular}{l|l||l|l} 
elemento & sigla & elemento & sigla \\
\hline \hline$P_{1} / P_{1}$ estabilizado & GLS & $P_{2} / P_{1}$ & TH2 \\
$P_{1}^{+} / P_{1}$ & $\mathrm{ME}$ & $P_{2}^{+} / P_{1}^{K}$ & $\mathrm{CR}$ \\
$P_{1}^{+} / P_{1}$ simplificado & $\mathrm{ME}-\mathrm{S}$ & $P_{2}^{+} / P_{1}^{K}$ simplificado & CR-S
\end{tabular}

O erros $\left\|\mathbf{u}-\mathbf{u}_{h}\right\|_{0}$ (erro da velocidade em $L^{2}$ ), $\left\|\nabla \mathbf{u}-\nabla \mathbf{u}_{h}\right\|_{0}$ (erro da velocidade em $\left.H^{1}\right),\left\|p-p_{h}\right\|_{0}$ (erro da pressão em $\left.L^{2}\right)$ e $\left\|\nabla p-\nabla p_{h}\right\|_{0}$ (erro da pressão em $H_{1}$ ) serão denotados por $E_{u}, E_{\nabla u}, E_{p}$ e $E_{\nabla p}$, respectivamente.

\subsection{Validações}

\subsubsection{Convergência espacial em 2D}

A fim de verificar a convergência espacial em 2D, é considerado um problema introduzido por Kovasznay [33], que consiste em modelar um escoamento laminar estacionário $\left(\partial_{t} \mathbf{u}=\mathbf{0}\right)$ sem o termo de força de campo $(\boldsymbol{g}=\mathbf{0})$. A velocidade $\mathbf{u}=\left(u_{1}, u_{2}\right)$ e a pressão $p$ exatas para este problema são dadas por

$$
\begin{aligned}
u_{1} & =1-\exp \left(a x_{1}\right) \cos \left(2 \pi x_{2}\right), \\
u_{2} & =\frac{a}{2 \pi} \exp \left(a x_{1}\right) \sin \left(2 \pi x_{2}\right), \\
p & =\frac{1}{2}\left(1-\exp \left(2 a x_{1}\right)\right),
\end{aligned}
$$

para $\boldsymbol{x}=\left(x_{1}, x_{2}\right) \in \mathbb{R}^{2}$, onde $a=1 /(2 \mu)-\sqrt{\left(1 /\left(4 \mu^{2}\right)+4 \pi^{2}\right)}$. O problema é resolvido numericamente em quatro malhas regulares, mantidas fixa no domínio $\Omega=$ $[-0.5,1] \times[-0.5,0.5]$ considerado, e constituídas de $9 \times 7,17 \times 13,33 \times 25$ e $65 \times 49$ nós, respectivamente. O número de Reynolds adotado é $R e=40$ (equivalente a $\rho=1$ e $\mu=0.025)$. Os elementos considerados são os quatro elementos introduzidos na Sec. (2.3), mais as versões não simplificadas (i.e., $P_{1}^{+} / P_{1}$ e $P_{2}^{+} / P_{1}^{K}$ sem eliminações estáticas), a fim de verificar a influência da simplificação na precisão. O fluido está inicialmente em repouso com condição de contorno de Dirichlet prescrita em todo bordo (por meio da solução analítica), exceto em $x=1$, onde é prescrita uma condição de Neumann (calculado com a solução analítica).

Em todos os casos, foram necessárias quatro iterações de Newton para se chegar à solução, exceto para os elementos quadráticos (TH2, CR e CR-S) com a malha mais 


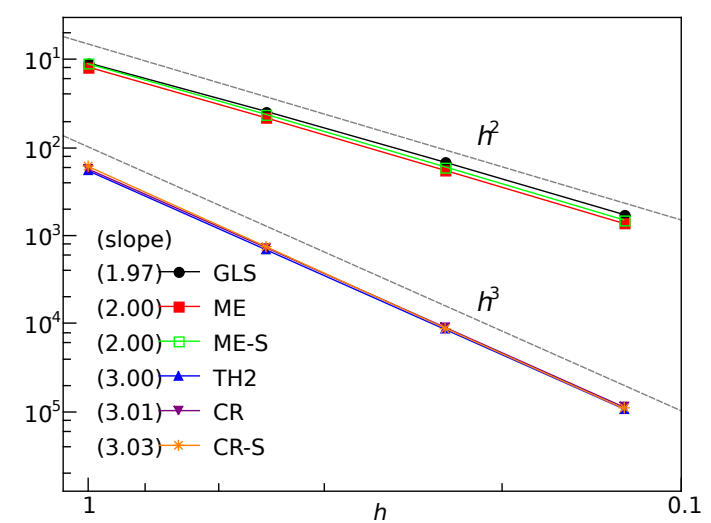

(a) Erro da velocidade em $L^{2}$.

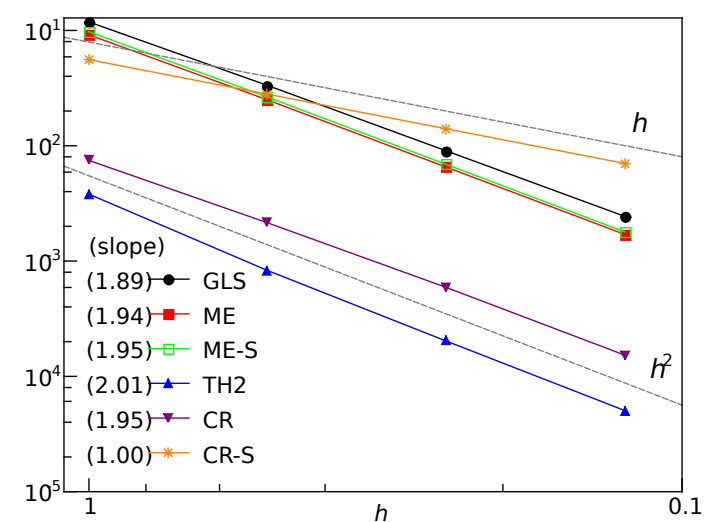

(c) Erro da pressão em $L^{2}$.

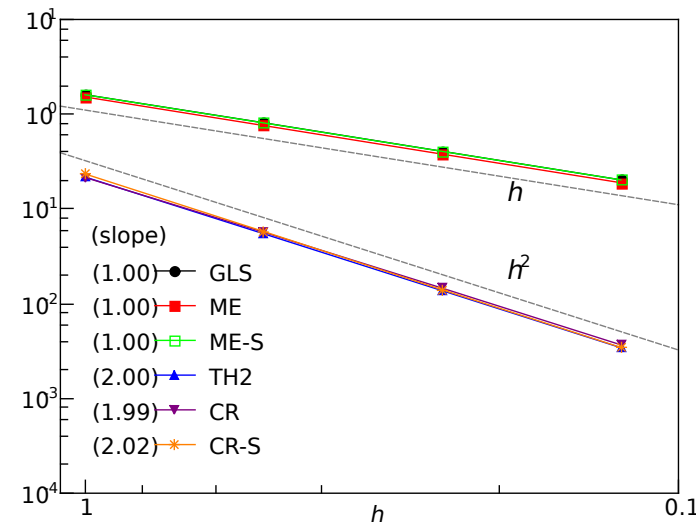

(b) Erro da velocidade em $H^{1}$.

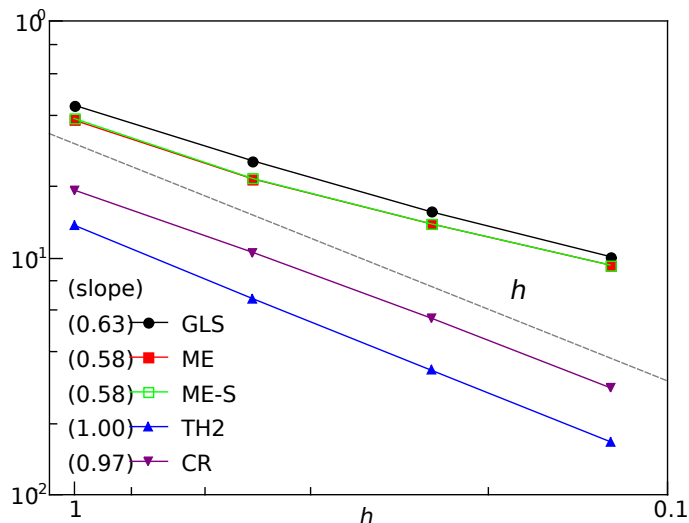

(d) Erro da pressão em $H^{1}$.

Figura 3.1: Erros da velocidade e pressão no problema de Kovasznay.

fina $65 \times 49$, onde o método de Newton divergiu. Nestes casos, usar ILU(1) em vez de ILU(0) resolveu o problema. Estes resultados, inclusive a questão da convergência com os elementos quadráticos, estão de acordo com os testes feitos em Codina et al. [14].

Os gráficos com os erros de discretização pelo tamanho da malha $h$ (artificial) podem ser encontrados na Fig. (3.1). Em todos os casos, é obtida convergência ótima, exceto para o gradiente da pressão com elementos lineares, nos quais a teoria não garante sua convergência. Os resultados obtidos na versão simplificada do mini-elemento mostrou-se muito semelhante à versão original, confirmando a premissa feita de que os coeficientes das funções bolhas são pequenos o suficiente para serem desprezados na contribuição do termo não linear (confirmado também em Soulaimani et al. [45]) e que também podem ser desprezados na interpolação final.

Comparando os elementos de Crouzeix-Raviart e Taylor-Hood de segunda ordem, nota-se que o último, apesar de possuir um espaço mais pobre tanto para pressão como para velocidade, apresenta erros menores na pressão e em seu gradiente. A explicação 
para estes resultados é dada em Chamberland et al. [12] (que obtiveram resultados semelhantes), que diz que "para um espaço fixo $W_{h}$, enriquecendo-se $Q_{h}$, diminui-se $\alpha$ (na condição inf-sup (2.25)) e isto pode acarretar consequências dramáticas na precisão da pressão", pois "o erro de discretização da pressão é proporcional à $1 / \alpha^{2}$ ".

Assim como no caso do ME, a versão simplificada de CR mostrou-se praticamente igual à versão original na aproximação da velocidade. O mesmo não aconteceu com a pressão apenas porque seu gradiente (estaticamente eliminado) não foi recuperado para efetuar a interpolação, o que levou à queda de um na ordem de convergência da pressão em $L^{2}$, como pode ser observado na Fig. (3.1c).

\subsubsection{Convergência temporal}

A fim de verificar a convergência temporal do método de Crank-Nicolson (CN) em uma formulação ALE, são considerados problemas com soluções manufaturadas u e $p$ pertencentes aos espaços discretos $W_{h}$ e $Q_{h}$, respectivamente, de modo que a única fonte de erro do problema seja a discretização temporal. Três famílias de problemas (A), (B) e (C) são consideradas, cujas velocidades $\mathbf{u}=\left(u_{1}, u_{2}\right)$ e pressões $p$ manufaturadas são construídas como

$$
\begin{aligned}
& \mathbf{u}_{A}=0.4\left(x_{2} \cos (\omega t), x_{1} \sin (\omega t)\right) \\
& \mathbf{u}_{B}=0.4 \cos (\omega t)\left(x_{2}, x_{1}\right) \\
& \mathbf{u}_{C}=1.0 \cos (\omega t)\left(x_{1},-x_{2}\right) \\
& p_{A}=p_{B}=p_{C}=0
\end{aligned}
$$

onde $\omega$ é uma frequência dada que assume um valor para cada teste. A densidade $\rho$ e a viscosidade $\mu$ são tomadas iguais a um, e as forças $\boldsymbol{g}$ correspondentes aos problemas (A), (B) e (C) são dadas por

$$
\begin{aligned}
& \boldsymbol{g}_{A}=-0.4 \omega\left(x_{2} \sin (\omega t),-x_{1} \cos (\omega t)\right)+0.16 \sin (\omega t) \cos (\omega t) \boldsymbol{x}, \\
& \boldsymbol{g}_{B}=-0.4 \omega \sin (\omega t)\left(x_{2}, x_{1}\right)+0.16 \cos ^{2}(\omega t) \boldsymbol{x}, \\
& \boldsymbol{g}_{C}=-0.4 \omega \sin (\omega t)\left(-x_{1}, x_{2}\right)+0.16 \cos ^{2}(\omega t) \boldsymbol{x} .
\end{aligned}
$$

A malha é movimentada apenas no nós internos sob uma velocidade imposta como

$$
\mathbf{v}=a \cdot \cos (\lambda t)(-1,1)
$$



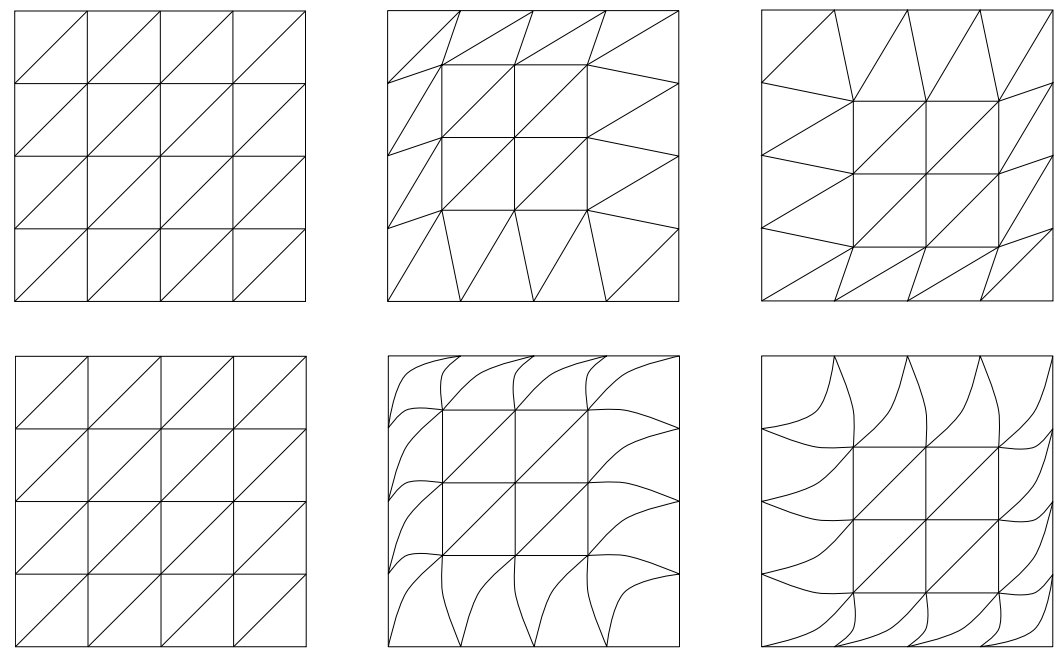

Figura 3.2: Movimento da malha nos testes de convergência temporal referente a velocidade em (3.6). Na primeira linha, a malha é movimentada sob deformações lineares ao passo que na segunda, a malha é movimentada sob deformações quadráticas.

onde $a$ e $\lambda$ assumem um valor diferente para cada teste. O movimento da malha descrito por tal velocidade por ser visto na Fig. (3.2).

Quandode outra forma, supõe-se que os testes são feitos no intervalo de tempo $[0,2]$ com o refinamento do tamanho do passo de tempo $\Delta t$ calculado como $\Delta t=2^{-k}$, $k=1,2, .$. , e os erros $L^{2}$ e $H^{1}$ são calculados como a média dos erros computados em cada passo de tempo $n$, ou seja,

$$
E=\frac{1}{N} \sum_{n=0}^{N}\left\|w\left(t^{n}\right)-w_{h}\left(t^{n}\right)\right\|_{L^{2} \text { ou } H^{1}}
$$

onde $N$ é um inteiro tal que $N \cdot \Delta t=2$.

\section{Convergência em malha fixa}

A fim de validar o método de CN e também verificar a melhor opção entre computar a pressão no tempo $n+1$ ou $n+1 / 2$ na formulação variacional discretizada (rever Eq. (2.42)), o problema (A) foi considerado com os valores $\omega=\lambda=\pi$ e $a=0$ (malha fixa), utilizando-se o elemento TH2. Tanto a velocidade (não ilustrada) como a pressão (ver Fig. (3.3)) convergiram quadraticamente como predito pela teoria. Quando usado a pressão no passo $n+1$ em vez de no passo $n+1 / 2$, houve um ligeiro ganho na precisão da pressão e nenhuma mudança na precisão da velocidade, de modo que não há diferença significativa entre escolher um e outro. Neste trabalho, é escolhido computar a pressão no passo $n+1$, apenas porque dessa forma não é necessário especificar sua condição inicial $p(\boldsymbol{x}, t=0)$. 

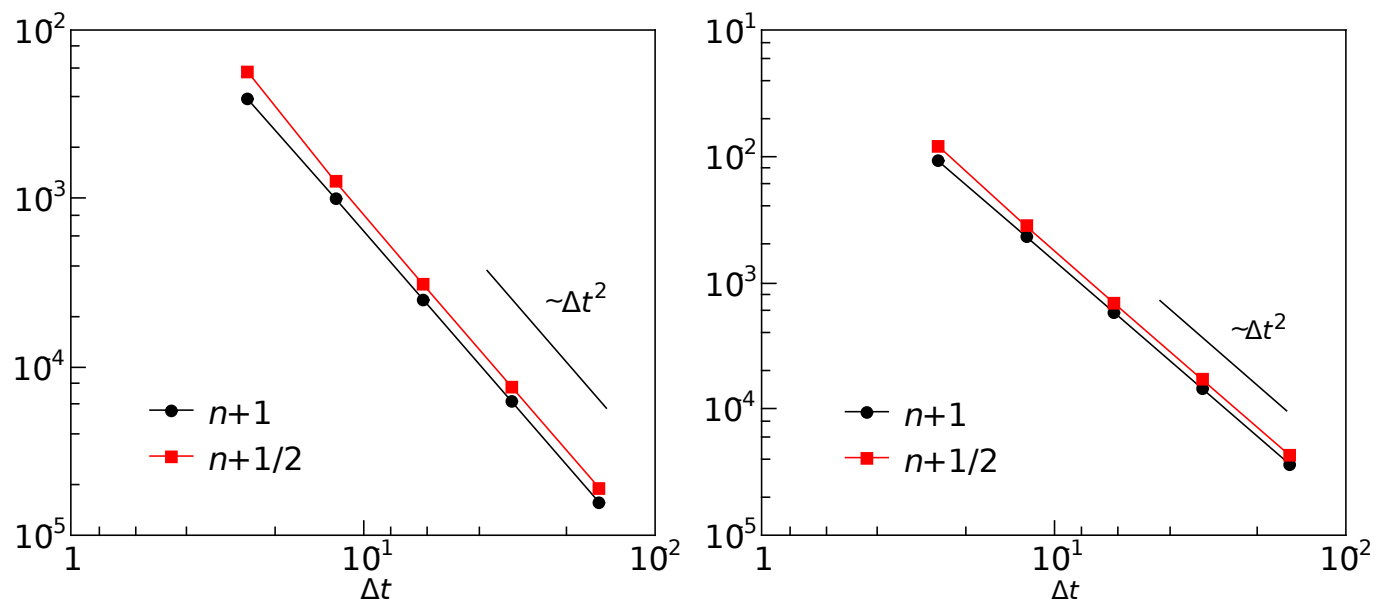

Figura 3.3: Comparação dos erros da pressão em $L^{2}$ (esquerda) e $H^{1}$ (direita) para o método de Crank-Nicolson em uma malha fixa entre a pressão computada no passo de tempo $n+1$ e a computada em $n+1 / 2$.

\section{Convergência em malha dinâmica - I}

Para o segundo teste, também com o problema (A) utilizando o elemento TH2, foi escolhido $\omega=0$ (escoamento de Couette), $\lambda=\pi$ e $a=0.2$ e considerados vários refinamentos diferentes para a malha com vários intervalos de tempo $\Delta t$. Em todos os casos, foi obtida a solução exata (dentro da precisão de máquina), diferentemente do que é reportado em Étienne et al. [22] (também para um escoamente de Couette), que obteve apenas convergência linear para o CN na mesma formulação adotada neste trabalho (formulação não conservativa), mas que também obteve solução exata com a formulação conservativa.

\section{Convergência em malha dinâmica - II}

No terceiro teste, com o problema (A) e (B), foram escolhidos $\omega=\lambda=\pi$ e $a=0.2$, de forma que tanto a velocidade da malha como a velocidade do fluido fossem não estacionárias, representado assim um caso um pouco mais geral. Foram considerados os elementos GLS, ME-S e TH2. Este último foi considerado em dois casos distintos: em elementos isoparamétricos (i.e., deformações quadráticas) e em elementos subparamétricos (i.e., deformações lineares). Os resultados podem ser vistos na Fig. (3.4). Todos os erros, de ambos problemas (A) e (B), decaíram quadraticamente, exceto o erro da pressão em $L^{2}$ para o elemento TH2 no problema (A). A explicação para essa queda de convergência da pressão apenas para o elemento quadrático não foi encontrada, entretanto, sabe-se que o problema está diretamente relacionado com a forma da velocidade relativa $\mathbf{c}_{h}=\mathbf{u}_{h}-\mathbf{v}_{h}$, pois, quando a velocidade da malha é alterada 


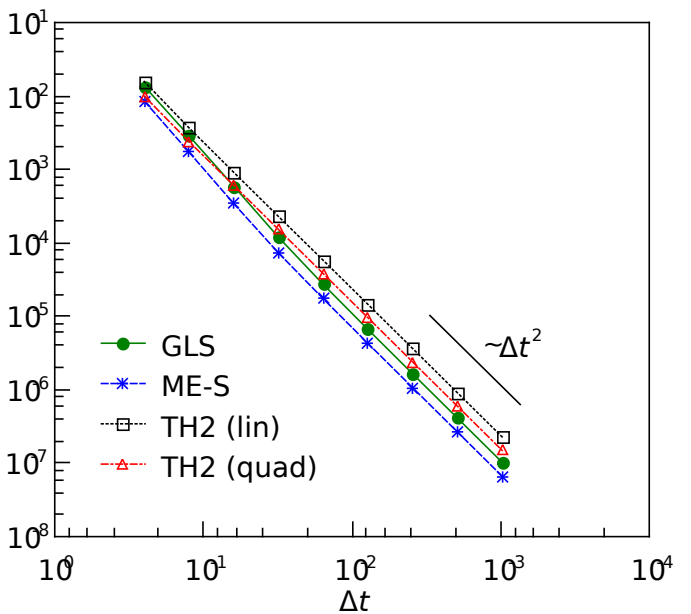

(a) Erro da velocidade em $H^{1}$ no prob. (A).

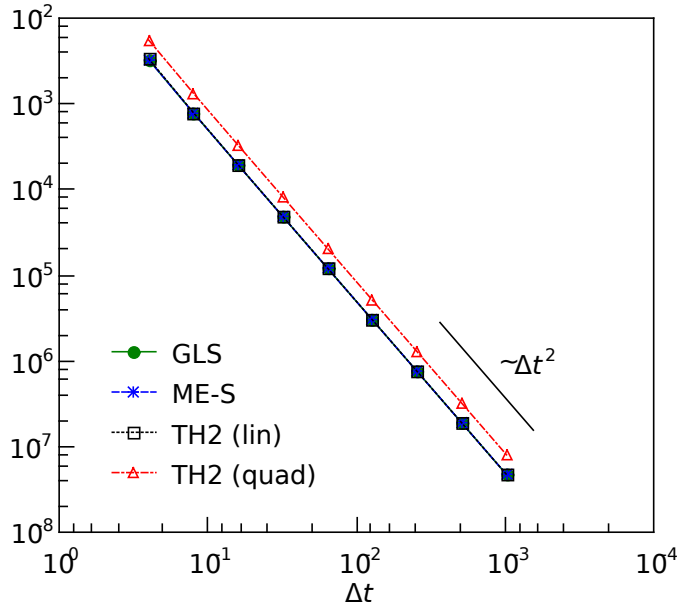

(c) Erro da velocidade em $H^{1}$ no prob. (B).

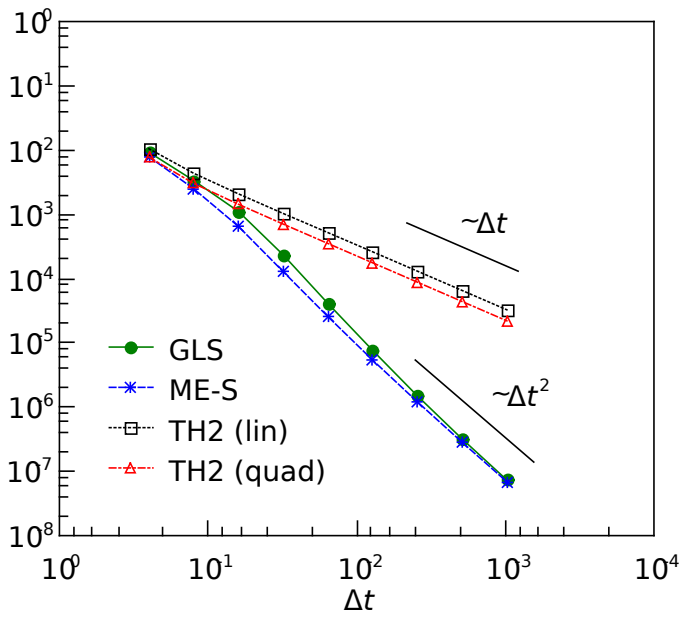

(b) Erro da pressão em $L^{2}$ no prob. (A).

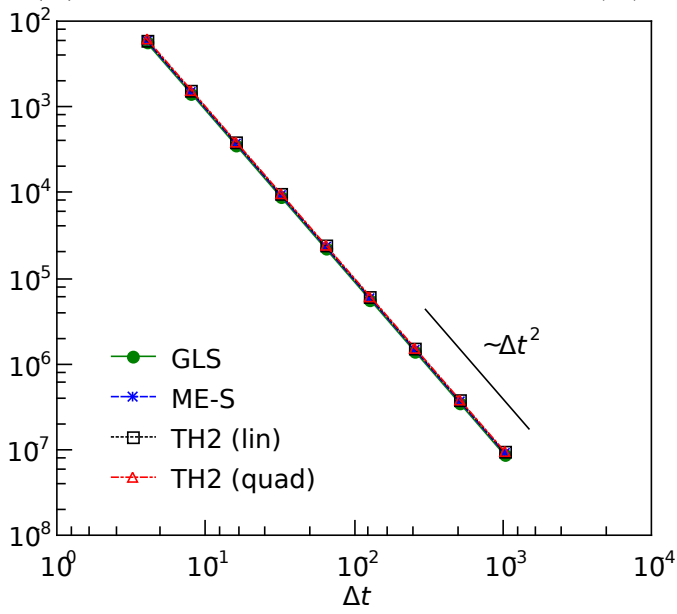

(d) Erro da pressão em $L^{2}$ no prob. (B).

Figura 3.4: Testes de convergência temporal dos problemas (A) e (B) na formulação ALE. As abreviações "lin" e "quad" significam elementos lineares e curvos, respectivamente.

de (3.6) para $\mathbf{v}=a \cdot \sin (\lambda t)(-1,1)$, o cenário se inverte: a pressão passa a convergir quadraticamente no problema (A) mas não em (B). Uma questão é se a convergência da velocidade também pode ser deteriorada para alguma velocidade da malha específica.

\section{Convergência em malha dinâmica - III}

No último teste, foi considerado o problema (C) com a frequência $\omega=\pi$. A velocidade da malha não é mais especificada, e sim dada pela estratégia vista no primeiro capítulo, onde a velocidade da malha $\mathbf{v}_{h}$ no tempo $t^{n+1 / 2}$ é dada pela diferença entre as posições nodais da malha suavizada em $n+1$ e as da malha em $n$, ou seja (rever (2.1)),

$$
\mathbf{v}_{h}^{n+1 / 2}=\frac{\text { Suavização }\left(\mathbf{x}_{h}^{n+1}\right)-\mathbf{x}_{h}^{n}}{\Delta t} .
$$



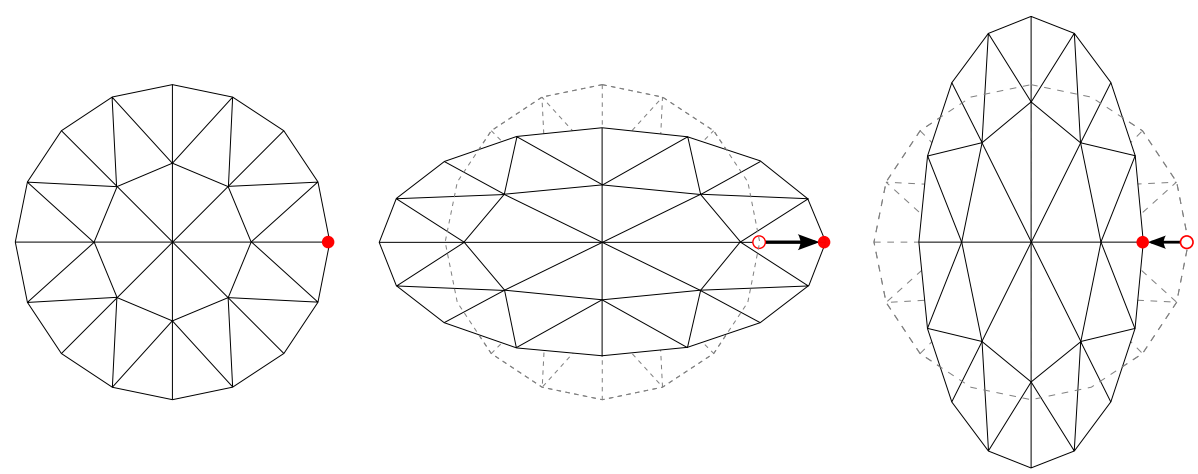

Figura 3.5: Movimentação da malha no teste de convergência das posições nodais. O nó ressaltado nas figuras é o nó acompanhado durante a simulação.

O propósito deste teste é verificar que o método de atualização das posições nodais

$$
\mathbf{x}_{h}^{n+1}=\mathbf{x}_{h}^{n+}+\Delta t\left[(\theta+1) \mathbf{u}_{h}^{n}-\theta \mathbf{u}_{h}^{n-1}\right]
$$

para $\theta=1 / 2$ (Adams-Bashforth), é suficiente para alcançar convergência quadrática (pelo menos nos testes considerados) nas posições nodais do bordo que dão forma ao domínio, sem precisar recorrer a uma estratégia mais complexa (e computacionalmente mais cara) como preditor-corretor (corrigir $\mathbf{x}_{h}^{n+1}$ com a solução encontrada $\mathbf{u}_{h}^{n+1}$ ) e métodos monolíticos, que acoplam $\mathbf{x}_{h}^{n+1}$ ao sistema de equações.

O domínio considerado é inicialmente um círculo de raio 1 centrado em $(0,0)$, onde em todo o contorno é imposta a condição de Neumann calculada a partir da solução exata. A normal n, necessária para computar a tração no termo da condição de contorno $\left(\int_{\Gamma} \mathbf{w}_{h} \cdot \boldsymbol{\sigma} \cdot \mathbf{n}\right)$, é calculada a partir da própria malha. Para analisar a convergência da posição da malha, um nó no contorno $\mathbf{q}_{h}$, que está inicialmente em $(1,0)$ (ver Fig. (3.5)), é acompanhado por toda simulação, e então feita a medida do erro $E_{\mathbf{x}}$ de sua posição com a expressão

$$
E_{\mathbf{x}}=\frac{1}{N} \sum_{k=0}^{N}\left|\mathbf{q}_{h}\left(t^{k}\right)-\mathbf{q}\left(t^{k}\right)\right|,
$$

onde $N$ é tal que $N \cdot \Delta t=$ tempo de simulação $=1$ e $\mathbf{q}$ é a posição exata deste nó, dada por

$$
\mathbf{q}(t)=\mathbf{q}(0) \exp (\sin (\omega t) / \omega)
$$

Outra medida de erro considerada foi o erro volumétrico $E_{\mathrm{vol}}$, que mede a porcentagem 

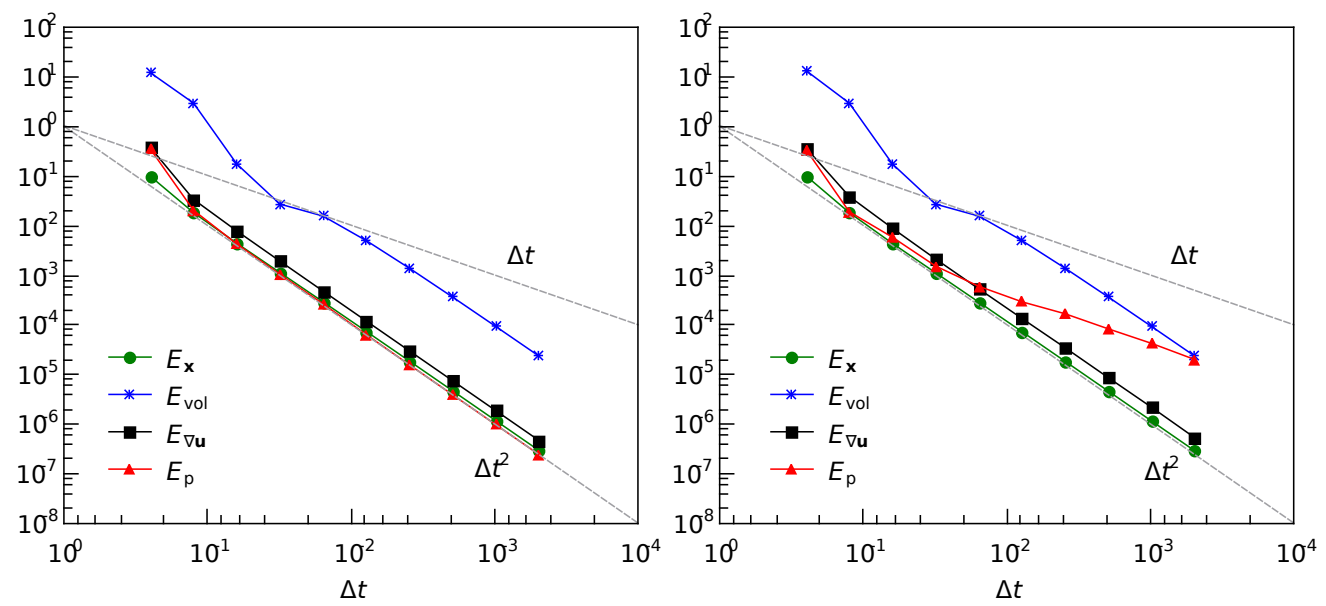

(a) Testes utilizando o elemento GLS com(b) Testes utilizando o elemento TH2 com atualizações dos nós da malha via atualizações dos nós da malha via Adams-Bashforth $(\theta=1 / 2$ em (3.8)). Adams-Bashforth $(\theta=1 / 2$ em (3.8)).

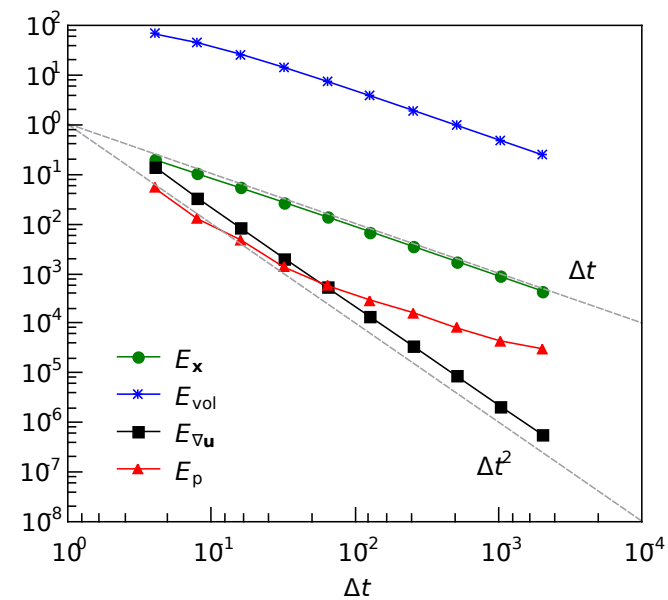

(c) Testes utilizando o elemento TH2 com atualizações dos nós da malha via Euler $(\theta=0 \mathrm{em}(3.8))$.

Figura 3.6: Testes de convergência das posições nodais da malha no problema (C).

com que o volume da malha é alterado durante a simulação. Sua expressão é dada por

$$
E_{\text {vol }}=100\left|\frac{\text { volume }_{\text {final }}-\text { volume }_{\text {inicial }}}{\text { volume }_{\text {inicial }}}\right| .
$$

Os passos de tempo escolhidos para analisar a convergência são os da sequência $\Delta t=$ $2^{-k}$, com $k=2$ até 11 , e o tempo de simulação é de 1 . Foram considerados para os testes, os elementos GLS e TH2, e dois métodos de atualização das posições nodais, Adams-Bashfort $(\theta=1 / 2$ em (3.8)) e Euler $(\theta=0$ em (3.8)).

Pelos resultados na Fig. (3.6), todos os casos que utilizavam Adams-Bashfort apre- 
sentaram convergência quadrática para a posição da malha, enquanto que com Euler foi obtida convergência apenas linear. A conservação de volume é outra propriedade bastante afetada pelo método de atualização do nós onde, mais uma vez, com AdamsBashfort, a convergência foi quadrática ao passo que em Euler foi apenas linear. Assim como já obervado nos outros testes, houve uma queda de convergência na pressão para o elemento quadrático TH2. 


\section{Capítulo 4}

\section{Elementos de geometria diferencial}

\subsection{Extensão e função distância}

Frequentemente, será necessário calcular o gradiente de funções que estão definidas somente em superfícies, como a normal e a tensão superficial, e por isso é conveniente definir uma extensão de tais funções para além da superfície. Seja $\Pi_{\Gamma}(\boldsymbol{x}): \mathcal{D} \rightarrow \Gamma$ a projeção normal de $\boldsymbol{x} \in \mathcal{D}$ em $\Gamma$, que coincide com o ponto da superfície mais próximo de $\boldsymbol{x}$, ou seja,

$$
\Pi_{\Gamma}(\boldsymbol{x})=\operatorname{argumento} \min _{\boldsymbol{y} \in \Gamma}\|\boldsymbol{y}-\boldsymbol{x}\|, \quad \boldsymbol{x} \in \mathcal{D},
$$

onde é assumido que $\Gamma$ seja regular o suficiente para que a normal $\mathbf{n}$ esteja definida em todos seus pontos, e que a região volumétrica finita $\mathcal{D} \supset \Gamma$ que envolve $\Gamma$ é tal que seja possível definir um único valor $\Pi_{\Gamma}(\boldsymbol{x})$ para cada $\boldsymbol{x}$ dessa região. A extensão normal de uma função $f$ definida pelo menos em $\Gamma$, é definida por

$$
\tilde{f}(\boldsymbol{x}) \doteq f\left(\Pi_{\Gamma}(\boldsymbol{x})\right) .
$$

É dito "pelo menos em $\Gamma$ ", pois a definição da extensão $\tilde{f}$ serve para o caso em que $f$ esteja definida também fora de $\Gamma$. A mesma definição é análoga para extensões de vetores ou tensores de qualquer ordem.

A função distância $\mathfrak{D}(\boldsymbol{x})$ está relacionada com a projeção normal da seguinte forma:

$$
\mathfrak{D}(\boldsymbol{x})=\left(\boldsymbol{x}-\Pi_{\Gamma}(\boldsymbol{x})\right) \cdot \tilde{\mathbf{n}}(\boldsymbol{x}), \quad \boldsymbol{x} \in \mathcal{D},
$$




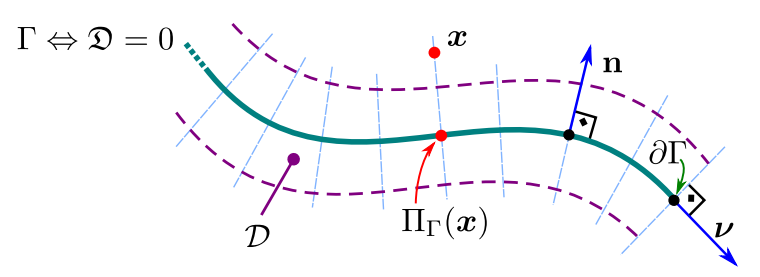

Figura 4.1: Ilustração de uma superfície $\Gamma$, da região $\mathcal{D}$ que a envolve, de sua fronteira $\partial \Gamma$, da normal $\mathbf{n}$, da conormal $\boldsymbol{\nu}$ e da projeção normal $\Pi_{\Gamma}$ de um ponto $\boldsymbol{x}$.

onde $\tilde{\mathbf{n}}$, seguindo a convenção, é a extensão de $\mathbf{n}$. Reciprocamente,

$$
\Pi_{\Gamma}(\boldsymbol{x})=\boldsymbol{x}-\mathfrak{D}(\boldsymbol{x}) \tilde{\mathbf{n}}(\boldsymbol{x}) .
$$

Outra igualdade útil, é

$$
\nabla \mathfrak{D}(\boldsymbol{x})=\tilde{\mathbf{n}}(\boldsymbol{x})
$$

Estes conceitos são ilustrados na Fig. (4.1).

\subsection{Operadores diferencias na superfície}

O gradiente de superfície, denotado por $\nabla_{\Gamma}$, de uma função $f: \mathcal{D} \rightarrow \mathbb{R}^{d}$, é dado por

$$
\nabla_{\Gamma} f(\boldsymbol{x})=\mathbf{P}(\boldsymbol{x}) \cdot \nabla f(\boldsymbol{x}), \quad \boldsymbol{x} \in \Gamma,
$$

onde $\mathbf{P}(\boldsymbol{x})=\mathbf{I}_{d}-\tilde{\mathbf{n}}(\boldsymbol{x}) \otimes \tilde{\mathbf{n}}(\boldsymbol{x})$ é a matriz de projeção. Se no caso anterior $f=\tilde{f}$, então $\nabla_{\Gamma} f(\boldsymbol{x})=\nabla f(\boldsymbol{x})$. No caso do gradiente de superfície de um vetor $\mathbf{w}: \Gamma \rightarrow \mathbb{R}^{d}$, tem-se que

$$
\nabla_{\Gamma} \mathbf{w}=\nabla \mathbf{w} \cdot \mathbf{P}=\nabla \tilde{\mathbf{w}}
$$

Também é definido o divergente superficial de um campo w:

$$
\nabla_{\Gamma} \cdot \mathbf{w}=\mathbf{P}: \nabla \mathbf{w}=\nabla \cdot \tilde{\mathbf{w}}
$$

Finalmente, outro operador muito utilizado é o laplaciano de superfície, $\nabla_{\Gamma}^{2}=\nabla_{\Gamma} \cdot \nabla_{\Gamma}$, que é conhecido na literatura como operador de Laplace-Beltrami. 


\subsection{Curvaturas e formas fundamentais}

Seja $(\boldsymbol{\Phi}, \hat{K})$ uma parametrização local de $K \subset \Gamma$, onde

$$
\begin{aligned}
\boldsymbol{\Phi}: \hat{K} \subset \mathbb{R}^{d-1} & \longrightarrow K=\boldsymbol{\Phi}(\hat{K}), \\
\boldsymbol{y} & \longmapsto \boldsymbol{x}=\boldsymbol{\Phi}(\boldsymbol{y}), \quad \boldsymbol{y} \in \hat{K}, \boldsymbol{x} \in K .
\end{aligned}
$$

Seja $\mathbb{M}_{m \times n}$ o conjunto das matrizes reais de tamanho $m$ por $n$ e $\mathbf{F}(\boldsymbol{y}) \in \mathbb{M}_{d \times(d-1)}$ a matriz jacobiana da transformação $\boldsymbol{\Phi}(\boldsymbol{y})$, que pode ser escrita como

$$
\mathbf{F}(\boldsymbol{y}) \doteq \nabla_{\boldsymbol{y}} \boldsymbol{\Phi}(\boldsymbol{y}) \doteq \frac{\partial \boldsymbol{\Phi}}{\partial \boldsymbol{y}} \doteq\left[\frac{\partial \Phi_{i}}{\partial y_{j}}\right]
$$

A primeira forma fundamental de $\Gamma$ (ou tensor métrico de $\Gamma$ ) $\mathbf{G}=\mathbf{G}(\boldsymbol{y}), \boldsymbol{y} \in \hat{K}$, é definida como

$$
\mathbf{G} \doteq \mathbf{F}^{T} \mathbf{F}
$$

O tensor $\mathbf{G}$ é sempre simétrico e positivo definido, uma vez que $\mathbf{F}$ tem posto completo, portanto, é possível definir a pseudoinversa de $\mathbf{F}$, denotada por $\mathbf{F}^{+}$, como sendo

$$
\mathbf{F}^{+} \doteq \mathbf{G}^{-1} \mathbf{F}^{T}
$$

Mais adiante, será utilizado o seguinte lema:

Lema 4.1 (Pseudoinversas): As matrizes $\mathbf{F}$ e $\mathbf{F}^{+}$satisfazem

$$
\begin{aligned}
& \mathbf{F}^{+} \mathbf{F}=\mathbf{I}_{d-1}, \\
& \mathbf{F F}^{+}=\mathbf{P} \circ \boldsymbol{\Phi}=\mathbf{I}_{d}-\hat{\mathbf{n}} \otimes \hat{\mathbf{n}} .
\end{aligned}
$$

onde $\hat{\mathbf{n}}(\boldsymbol{y}) \doteq \mathbf{n} \circ \boldsymbol{\Phi}$.

Prova: Pode ser encontrada em Pauletti [37].

Outro tensor importante na geometria diferencial é a segunda forma fundamental, denotada aqui por $\mathbf{H}$, e definida por

$$
\mathbf{H}(\boldsymbol{x}) \doteq \nabla_{\Gamma} \mathbf{n}(\boldsymbol{x}), \quad \boldsymbol{x} \in K .
$$

Este é um tensor simétrico, pois

$$
\nabla_{\Gamma} \mathbf{n}=\nabla \tilde{\mathbf{n}}=\nabla \nabla \mathfrak{D}(\boldsymbol{x})=\left[\partial_{i j} \mathfrak{D}(\boldsymbol{x})\right]
$$


e possui o versor normal $\mathbf{n}$ como um autovetor associado a um autovalor nulo, pois $\partial_{j} \tilde{n}_{i} n_{j}=0$, por construção.

Observação 4.1: Muitas vezes na literatura, a segunda forma fundamental é referida como sendo o tensor $\left.\mathbf{H}(\boldsymbol{y})\right|_{i j}=\hat{\mathbf{n}} \cdot \partial_{i j} \boldsymbol{\Phi}$, com $i, j=1, . ., d-1$ (e portanto $\mathbf{H}(\boldsymbol{y}) \in$ $\left.\mathbb{M}_{(d-1) \times(d-1)}\right)$.

As quantidades importantes relacionadas à segunda forma fundamental são suas invariantes (ver sobre invariantes de tensores em Gurtin et al. [29]). No caso $d=3$, denotando por $\kappa_{1}(\boldsymbol{x})$ e $\kappa_{2}(\boldsymbol{x})$ os outros autovalores de $\mathbf{H}$ (chamados de curvaturas principais), as invariantes de $\mathbf{H}$, denotadas por $I_{k}(\mathbf{H}), k=1,2$ e 3, são dadas por

$$
\begin{aligned}
& I_{1}(\mathbf{H})=\operatorname{tr}(\mathbf{H})=\kappa_{1}+\kappa_{1}, \\
& I_{2}(\mathbf{H})=\frac{1}{2}\left[\operatorname{tr}^{2}(\mathbf{H})-\operatorname{tr}\left(\mathbf{H}^{2}\right)\right]=\kappa_{1} \kappa_{2}, \\
& I_{3}(\mathbf{H})=\operatorname{det}(\mathbf{H})=0 .
\end{aligned}
$$

As invariantes de um tensor, em especial para o $\mathbf{H}$, satisfazem

$$
I_{k}(\mathbf{H})=I_{k}\left(\mathbf{Q H Q}^{T}\right), \quad \forall \mathbf{Q} \in \mathbb{M}_{d \times d} \text { ortogonal, }
$$

e por isso elas são iguais em qualquer sistema de coordenadas cartesiano. As duas primeiras invariantes, conhecidas como curvatura média e curvatura gaussiana, são denotadas aqui por, respectivamente,

$$
\begin{aligned}
\kappa & \doteq \kappa_{1}+\kappa_{2}, \\
\kappa_{G} & \doteq \kappa_{1} \kappa_{2} .
\end{aligned}
$$

Vale citar o notável teorema Egregium de Gauss, que diz que a curvatura Gaussiana é invariante por isometria.

\subsection{Outros fatos e definições}

Seja $\mathbf{i d}_{\Gamma}(\boldsymbol{x})$ a função identidade em $\Gamma$. Seguem as seguintes igualdades. 
Lema 4.2 (Função identidade):

$$
\begin{aligned}
\tilde{\mathbf{i d}}_{\Gamma}(\boldsymbol{x}) & =\Pi_{\Gamma}(\boldsymbol{x}), \quad \boldsymbol{x} \in \mathcal{D} ; \\
\nabla_{\Gamma} \mathbf{i} \mathbf{d}_{\Gamma}(\boldsymbol{x}) & =\mathbf{P}=\mathbf{I}_{d}-\mathbf{n} \otimes \mathbf{n} ; \\
\nabla_{\Gamma}^{2} \mathbf{i} \mathbf{d}_{\Gamma}(\boldsymbol{x}) & =-\kappa \mathbf{n} .
\end{aligned}
$$

Prova: Pode ser encontrada em Buscaglia e Ausas [10].

A extensão do lema da integral por partes para superfícies é dada pelo seguinte lema:

Lema 4.3 (Integral por partes em superfícies): Seja $f$ e q uma função escalar e vetorial, respectivamente, definidas e diferenciáveis em $\Gamma$.

$$
\begin{gathered}
\int_{\Gamma} \nabla_{\Gamma} f=\int_{\Gamma} f \kappa \mathbf{n}+\int_{\partial \Gamma} f \boldsymbol{\nu} \\
\int_{\Gamma} \nabla_{\Gamma} \cdot \mathbf{q}=\int_{\Gamma} \kappa \mathbf{n} \cdot \mathbf{q}+\int_{\partial \Gamma} \mathbf{q} \cdot \boldsymbol{\nu}
\end{gathered}
$$

Prova: Pode ser encontrada em Buscaglia e Ausas [10].

\subsection{Jacobiana de transformações em integrais}

O MEF aplicado aos problemas tratados neste trabalho resulta em integrais de funções escalares e vetoriais em variedades, por isso, são apresentados nesta subseção ferramentas para lidar com tais integrais de forma genérica, que são úteis não somente no contexto prático (montagem no MEF) como também no teórico (calcular derivadas de forma).

Considere uma função escalar $f: K \rightarrow \mathbb{R}$ suposta suave, onde $K$ é uma variedade de dimensão $m$ imersa em $\mathbb{R}^{d}$, com $m \leq d$, restrita aos casos de interesse $m \geq 1$ e $d \leq 3$. Assim, $K$ pode ser um curva imersa em $\mathbb{R}^{3}$, ou uma superfície em $\mathbb{R}^{3}$, e assim por diante. Considere a parametrização $(\boldsymbol{\Phi}, \hat{K})$ análoga à feita anteriormente, ou seja,

$$
\begin{aligned}
\boldsymbol{\Phi}: \hat{K} \subset \mathbb{R}^{m} \longrightarrow K & =\boldsymbol{\Phi}(\hat{K}), \quad K \subset \mathbb{R}^{d}, \\
\boldsymbol{y} & \longmapsto \boldsymbol{x}=\boldsymbol{\Phi}(\boldsymbol{y}), \quad \boldsymbol{y} \in \hat{K}, \boldsymbol{x} \in K .
\end{aligned}
$$

Uma integral do tipo integral $\int_{K} f(\boldsymbol{x})$ pode ser transformada na integral

$$
\int_{\hat{K}}(f \circ \boldsymbol{\Phi}) \sqrt{\operatorname{det} \mathbf{G}}
$$


onde $\mathbf{G}$ é a primeira forma fundamental. Note que a integral anterior é bastante natural no cálculo de um elemento no contexto do $\mathrm{MEF}$, pois $\boldsymbol{\Phi}(\boldsymbol{y})$ seria a transformação de um elemento unitário $\hat{K}$ (ou elemento mestre, ou elemento de referência) para o elemento real $K$. Por exemplo, seja $\mathbf{x}^{k}, k=1, . ., n$, os vértices do polítopo que representa o elemento e $\phi^{k}(\boldsymbol{y})$ suas funções de forma (suponha uma base de lagrange, por simplicidade), com $\boldsymbol{y}$ sendo a coordenada no elemento unitário e $\boldsymbol{\Phi}(\boldsymbol{y})=\sum_{k} \mathbf{x}^{k} \phi^{k}(\boldsymbol{y})$ (como convencional). O termo $\sqrt{\operatorname{det} \mathbf{G}}$ em (4.23) é fácil de ser computado, pois basta calcular a matriz jacobiana $\mathbf{F}$ do modo padrão no $\mathrm{MEF}$ e calcular $\mathbf{G}$ com a fórmula (4.11). O procedimento, obviamente, vale para elementos curvos.

Integrais do tipo $\int_{K} \nabla_{K} f(\boldsymbol{x})$, onde o operador $\nabla_{K}$ é o gradiente em $\mathbb{R}^{d}$ projetado em $K$ (por exemplo, o gradiente superficial $\nabla_{\Gamma}$ quando $m=2$ e $d=3$ ), também podem ser calculadas facilmente. Tal gradiente aplicado à função $f(\boldsymbol{x})$ fica (Pauletti [37])

$$
\nabla_{K} f(\boldsymbol{x})=\left(\left(\mathbf{F}^{+}\right)^{T} \cdot \nabla_{\boldsymbol{y}}(f \circ \boldsymbol{\Phi})\right) \circ \boldsymbol{\Phi}^{-1}
$$

lembrando que $\mathbf{F}^{+}$é a pseudoinversa de $\mathbf{F}$, dada por (4.12), e portanto, a integral $\int_{K} \nabla_{K} f(\boldsymbol{x})$ pode ser calculada como

$$
\int_{\hat{K}}\left(\mathbf{F}^{+}\right)^{T} \cdot \nabla_{\boldsymbol{y}}(f \circ \mathbf{\Phi}) \sqrt{\operatorname{det} \mathbf{G}} .
$$

As extensões para os casos em que o integrando são vetores, gradientes de vetores, divergentes de vetores, etc., são fáceis de serem deduzidas. No contexto do MEF, tal integral também vale para elementos curvos.

Outra alternativa de se calcular o gradiente $\nabla_{K}$ seria estender a imagem de $\boldsymbol{\Phi}$ para além de $K$, diga-se, para um $K^{*}$, adicionando $d-m$ dimensões extras em $\hat{K}$, resultando em um $\hat{K}^{*}$, de modo que $K^{*}$ e $\hat{K}^{*}$ tenham dimensões iguais a $d$, possibilitando que a inversa do gradiente da nova transformação $\boldsymbol{\Phi}^{*}$ (i.e., a inversa de um $\mathbf{F}^{*}$ ) faça sentido, não necessitanto a recorrer a pseudoinversas. Por exemplo, suponha que $m=2$ e $d=3$ ( $K$ é uma superfície imersa em $\mathbb{R}^{3}$ ), então é possível definir uma extensão para $\boldsymbol{\Phi}$ como

$$
\tilde{\boldsymbol{\Phi}}(\boldsymbol{y}, \eta) \doteq \boldsymbol{\Phi}(\boldsymbol{y})+\eta \mathbf{n}(\boldsymbol{\Phi}(\boldsymbol{y})), \quad(\boldsymbol{y}, \eta) \in \hat{K}^{*}=\hat{K} \times \mathbb{R},
$$

onde $\eta \in \mathbb{R}$ é a nova coordenada adicionada e $\mathbf{n}(\boldsymbol{x})$ é a normal. 


\section{Capítulo 5}

\section{Forças capilares, molhamento e ângulo de contato}

\subsection{Introdução}

Neste capítulo, são estudados escoamentos incompressíveis envolvendo interfaces entre dois fluidos Newtonianos, isotérmicos e imiscíveis, assim como a linha de contato que é formada entre as duas fases e um substrato sólido. Os típicos cenários dos problemas considerados e as notações adotadas sobre o domínio espacial podem ser vistos na figura 5.1. Basicamente, os objetos de estudo são gotas livres ou gotas que se espalham em substratos sólidos, onde estão envolvidas três fases: líquida (a gota propriamente dita), gasosa e sólida, onde o domínio espacial de cada fase depende do tempo $t \geq 0 \in \mathbb{R}$. A fase gasosa $\left(\Omega_{g}=\Omega_{g}(t)\right)$ será considerada passiva, de forma que a pressão, a viscosidade e a densidade nessa fase sejam nulas a todo instante, o sólido será considerado fixo, impermeável e perfeitamente liso e a fase líquida $(\Omega=\Omega(t))$ será a única fase explicitamente representada computacionalmente.

Os objetivos deste capítulo são apresentar o problema em uma formulação variacional que descreva a influência das fronteiras na dinâmica do molhamento e apresentar métodos para resolvê-lo numericamente. 

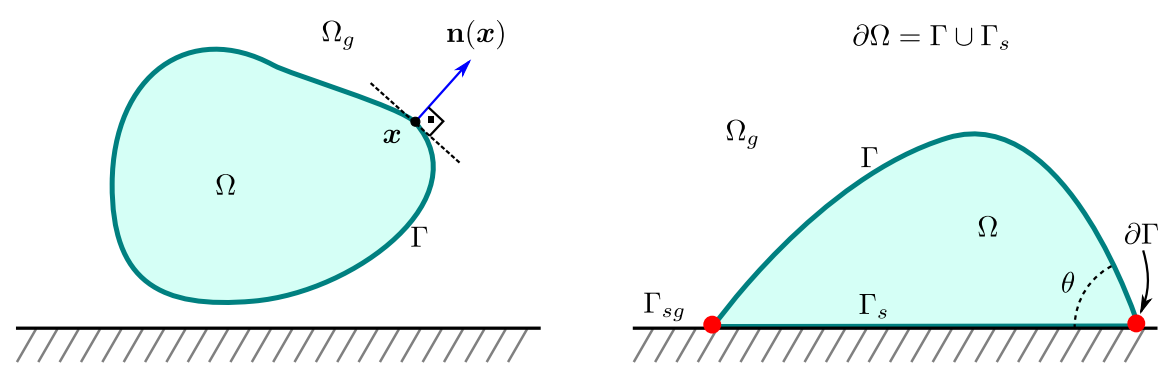

Figura 5.1: Na esquerda, está um caso de uma gota livre, enquanto que na direita, está um caso de uma gota em contato com um substrato sólido (a parte hachurada). Os pontos em vermelho representam a linha de contato que é denotada por $\partial \Gamma$.

\subsection{O princípio das potências virtuais}

Seguindo Buscaglia e Ausas [10], as forças capilares são introduzidas na formulação variacional do problema por meio de um princípio da mecânica conhecido como princípio das potências virtuais (Gurtin et al. [29]). Basicamente, este princípio diz que a soma da potência virtual interna com a potência de inércia deve ser igual à potência das forças externas, isto é,

$$
\int_{\Omega} \sigma: D \mathbf{w}+\int_{\Omega} \rho \frac{\mathrm{d} \mathbf{u}}{\mathrm{d} t} \cdot \mathbf{w}=\mathcal{P}(\mathbf{w}), \quad \forall \mathbf{w} \in W_{0}
$$

onde $\sigma, \rho, \mathbf{u}, p$ (contida no tensor $\sigma$ ) e $D$ seguem as definições do primeiro capítulo, w (a velocidade virtual) é um vetor do espaço de velocidades cinematicamente admissíveis $W_{0}$ (espaços dos campos solenoidais que satisfazem as condições de fronteira) e $\mathcal{P}(\mathbf{w})$ engloba as potências virtuais externas lineares em $\mathbf{w}$. O termo $\mathcal{P}(\mathbf{w})$ pode ser decomposto em três parcelas, uma de contribuição volumétrica, outra superficial e outra da linha de contato, como se segue

$$
\mathcal{P}(\mathbf{w})=\mathcal{P}_{\Omega}(\mathbf{w})+\mathcal{P}_{\Gamma}(\mathbf{w})+\mathcal{P}_{\partial \Gamma}(\mathbf{w})
$$

onde em $\mathcal{P}_{\Omega}(\mathbf{w})$, está a potência virtual da força de campo $\rho \boldsymbol{g}$, i.e.,

$$
\mathcal{P}_{\Omega}(\mathbf{w})=\int_{\Omega} \rho \boldsymbol{g} \cdot \mathbf{w} .
$$

Note que se $\rho \boldsymbol{g}$ é uma (densidade de) força conservativa, então ela pode se escrita como o negativo do gradiente de uma (densidade de) energia potencial $\Psi=\Psi(\boldsymbol{x}, t)$, i.e., $\rho \boldsymbol{g}=-\nabla \Psi$. Portanto,

$$
\mathcal{P}_{\Omega}(\mathbf{w})=-\int_{\Omega} \nabla \Psi \stackrel{*}{=}-\left.\frac{\mathrm{d}}{\mathrm{d} \varepsilon}\right|_{\varepsilon=0} \int_{\Omega_{\mathbf{w}, \varepsilon}} \Psi,
$$


onde em $*$ foi usado que $\nabla \cdot \mathbf{w}=0$, e onde $\Omega_{\mathbf{w}, \varepsilon}$ é o domínio $\Omega$ pertubado pelo campo de deformações $\varepsilon \mathbf{w}(\boldsymbol{x})$, ou seja,

$$
\Omega_{\mathrm{w}, \varepsilon}=\{\boldsymbol{y} \mid \boldsymbol{y}=\boldsymbol{x}+\varepsilon \mathbf{w}, \boldsymbol{x} \in \Omega\} .
$$

A demonstração da passagem em $*$, da direita para esquerda, é análogo ao do conhecido teorema de transporte de Reynolds. Definindo $\mathcal{E}(\Omega) \doteq \int_{\Omega} \Psi$, então, reconhece-se de (5.3) que a potência virtual $\mathcal{P}_{\Omega}(\mathbf{w})$ é a variação virtual desta energia total $\mathcal{E}$ sob o parâmetro $\varepsilon$ (dimensão de tempo) e o campo de velocidade virtual w, ou seja,

$$
\mathcal{P}_{\Omega}(\mathbf{w})=-\left.\frac{\mathrm{d}}{\mathrm{d} \varepsilon}\right|_{\varepsilon=0} \mathcal{E}\left(\Omega_{\mathbf{w}, \varepsilon}\right)
$$

A ideia é derivar a potência virtual associada às forças de superfície e às forças na linha de contato (forças capilares) por meio de uma energia, usando uma fórmula análoga à anterior para superfície. Uma vez encontrada esta potência, é fácil de se ver a relação do princípio das potências virtuais (5.1) com a formulação variacional no método de elementos finitos $(\mathbf{w}=\delta \mathbf{u}$ passa a ser a função teste). Potências virtuais de forças dissipativas são introduzidas postuladamente na formulação variacional.

\subsection{Potências virtuais na superfície}

A tensão superficial $\gamma$ é um campo escalar que pode ser interpretado como a energia que seria necessária fornecer às superfícies às quais ele está associado para que estas aumentassem sua área em uma unidade (de Gennes et al. [17]). Tais superfícies, cuja espessura é dada por flutuações da ordem de angstroms, se comportam basicamente como uma membrana elástica. A origem física da tensão superficial e mais esclarecimentos sobre sua interpretação podem ser encontradas em de Gennes et al. [17] e Bruus [9].

Considere por enquanto o caso em que $\partial \Gamma, \Gamma_{s}=\emptyset$ (não há linhas de contato e $\Gamma$ é uma superfície fechada). Dada a interpretação de $\gamma$ no parágrafo anterior, é natural supor uma energia para a superfície $\Gamma$ como

$$
\mathcal{E}(\Gamma) \doteq \int_{\Gamma} \gamma(\boldsymbol{x})
$$

A variação espacial de $\gamma$ é eventualmente relacionada com a concentração de surfactantes* e a temperatura em cada partícula material em $\boldsymbol{x}$. É possível mostrar que de fato

*Substâncias constituídas por moléculas anfifílicas (moléculas que apresentam a característica de possuírem uma região hidrofílica, e uma região hidrofóbica). 
esse modelo para energia leva ao modelo padrão para as forças superficiais. A potência virtual $\mathcal{P}_{\Gamma}(\mathbf{w})$ associada à essa energia é dada por

$$
\mathcal{P}_{\Gamma}(\mathbf{w})=-\left.\frac{\mathrm{d}}{\mathrm{d} \varepsilon}\right|_{\varepsilon=0} \mathcal{E}\left(\Gamma_{\mathbf{w}, \varepsilon}\right),
$$

onde $\Gamma_{\mathbf{w}, \varepsilon}=\{\boldsymbol{y} \mid \boldsymbol{y}=\boldsymbol{x}+\varepsilon \mathbf{w}, \boldsymbol{x} \in \Gamma\}$. O negativo do lado direito da igualdade anterior, geralmente denotada por

$$
\left.\mathrm{d} \mathcal{E}(\Gamma ; \mathbf{w}) \doteq \frac{\mathrm{d}}{\mathrm{d} \varepsilon}\right|_{\varepsilon=0} \mathcal{E}\left(\Gamma_{\mathbf{w}, \varepsilon}\right),
$$

é conhecido como a derivada de forma do funcional $\mathcal{E}(\Gamma)$ na direção do campo vetorial w. A seguir, será mostrado uma alternativa para se calcular esta derivada, porém, sem entrar em detalhes. Para passagens com rigor matemático é possível recorrer aos livros de otimização de forma, como, por exemplo, Delfour e Zolésio [18]. A demonstração que segue utiliza as ferramentas matemáticas do capítulo anterior.

Proposição 5.1: Supondo condições de regularidade suficientes sobre $\gamma$ e $\Gamma$, a derivada de forma da energia $\mathcal{E}(\Gamma)$ é dada por

$$
\mathrm{d} \mathcal{E}(\Gamma ; \mathbf{w})=\int_{\Gamma} \gamma \nabla_{\Gamma} \cdot \mathbf{w}
$$

Prova (Esboço): Definindo uma parametrização $\left(\boldsymbol{\Phi}_{\varepsilon}, \hat{\Gamma}\right)$ de $\Gamma_{\mathbf{w}, \varepsilon}$, onde

$$
\begin{aligned}
\boldsymbol{\Phi}_{\varepsilon}: \hat{\Gamma} \subset \mathbb{R}^{d-1} & \longrightarrow \Gamma_{\mathbf{w}, \varepsilon}=\boldsymbol{\Phi}_{\varepsilon}(\hat{\Gamma}), \\
\boldsymbol{y} & \longmapsto \boldsymbol{x}=\boldsymbol{\Phi}_{\varepsilon}(\boldsymbol{y}), \quad \boldsymbol{y} \in \hat{\Gamma}, \boldsymbol{x} \in \Gamma_{\mathbf{w}, \varepsilon},
\end{aligned}
$$

e denotando por $\mathbf{F}(\boldsymbol{y})$ e $\mathbf{G}(\boldsymbol{y})$ a matriz jacobiana e a primeira forma fundamental, respectivamente, tem-se, seguindo a fórmula (4.23), que

$$
\begin{aligned}
\mathrm{d} \mathcal{E}(\Gamma ; \mathbf{w})=\left.\frac{\mathrm{d}}{\mathrm{d} \varepsilon}\right|_{\varepsilon=0} \mathcal{E}\left(\Gamma_{\mathbf{w}, \varepsilon}\right) & =\left.\frac{\mathrm{d}}{\mathrm{d} \varepsilon}\right|_{\varepsilon=0} \int_{\Gamma_{\mathbf{w}, \varepsilon}} \gamma(\boldsymbol{x}) \\
& =\left.\frac{\mathrm{d}}{\mathrm{d} \varepsilon}\right|_{\varepsilon=0} \int_{\hat{\Gamma}}\left(\gamma \circ \boldsymbol{\Phi}_{\varepsilon}\right) J .
\end{aligned}
$$

onde $J(\boldsymbol{y}) \doteq \sqrt{\operatorname{det} \mathbf{G}(\boldsymbol{y})}$. A derivada $\mathrm{d} / \mathrm{d} \varepsilon$ pode entrar na integral, uma vez que o domínio $\hat{\Gamma}$ não depende de $\varepsilon$, portanto

$$
\mathrm{d} \mathcal{E}(\Gamma ; \mathbf{w})=\int_{\hat{\Gamma}}\left[\frac{\mathrm{d}}{\mathrm{d} \varepsilon}\left(\gamma \circ \boldsymbol{\Phi}_{\varepsilon}\right) J+\left(\gamma \circ \boldsymbol{\Phi}_{\varepsilon}\right) \frac{\mathrm{d}}{\mathrm{d} \varepsilon} J\right]_{\varepsilon=0} .
$$

O termo $\mathrm{d}\left(\gamma \circ \boldsymbol{\Phi}_{\varepsilon}\right) / \mathrm{d} \varepsilon$ é nulo, pois o valor de $\gamma$ em cada partícula deve permanecer o 
mesmo durante sua trajetória virtual, isso porque durante um movimento virtual não se pode contabilizar a troca de calor (do gradiente de temperatura) nem a variação de potencial químico (do gradiente de surfactantes) na variação de energia. O termo $\mathrm{d} J / \mathrm{d} \varepsilon$ pode ser com calculado com a ajuda da igualdade (Scovazzi e Hughes [41])

$$
\mathrm{d}(\operatorname{det} \mathbf{M})=\operatorname{det} \mathbf{M} \operatorname{tr}\left(\mathrm{d} \mathbf{M} \cdot \mathbf{M}^{-1}\right), \quad \mathbf{M} \in \mathbb{M}_{n \times n} \text { inversível, }
$$

onde tr é o traço. Trocando $\mathbf{M}$ por $\mathbf{G}$ na igualdade acima e lembrando que $\mathbf{G}=\mathbf{F}^{T} \mathbf{F}$, pode-se chegar em

$$
\frac{\mathrm{d}}{\mathrm{d} \varepsilon} J=\frac{1}{2 J} \frac{\mathrm{d}}{\mathrm{d} \varepsilon} \operatorname{det} \mathbf{G} \stackrel{(5.14)}{=} \frac{1}{2 J} J^{2} \operatorname{tr}\left[\frac{\mathrm{d}}{\mathrm{d} \varepsilon}\left(\mathbf{F}^{T} \mathbf{F}\right) \cdot\left(\mathbf{F}^{T} \mathbf{F}\right)^{-1}\right] .
$$

A derivada da matriz jacobiana $\mathbf{F}$ é o gradiente do campo w, isto é

$$
\frac{\mathrm{d}}{\mathrm{d} \varepsilon} \mathbf{F}=\nabla_{\boldsymbol{y}}\left(\mathbf{w} \circ \boldsymbol{\Phi}_{\varepsilon}\right)
$$

Finalmente, usando a regra da derivada do produto, usando a propriedade de gradiente superficial em (4.24) e o fato que $\operatorname{tr}(\mathrm{A} \cdot \mathrm{B})=\operatorname{tr}(\mathrm{B} \cdot \mathrm{A})$, obtém-se

$$
\frac{\mathrm{d}}{\mathrm{d} \varepsilon} J=\frac{1}{2} J \operatorname{tr}\left[\nabla_{\Gamma} \mathbf{w}+\left(\nabla_{\Gamma} \mathbf{w}\right)^{T}\right] \circ \boldsymbol{\Phi}_{\varepsilon}=J\left(\nabla_{\Gamma} \cdot \mathbf{w}\right) \circ \boldsymbol{\Phi}_{\varepsilon} .
$$

Substituindo a expressão acima em (5.13), chega-se no resultado esperado:

$$
\mathrm{d} \mathcal{E}(\Gamma ; \mathbf{w})=\int_{\hat{\Gamma}}\left[\left(\gamma \nabla_{\Gamma} \cdot \mathbf{w}\right) \circ \mathbf{\Phi}_{\varepsilon} J\right]_{\varepsilon=0}=\int_{\Gamma} \gamma \nabla_{\Gamma} \cdot \mathbf{w},
$$

que é igual ao negativo da potência virtual $\mathcal{P}(\mathbf{w})$.

Esta expressão ainda pode ser integrada por partes via (4.21b) para se obter sua forma mais conhecida:

$$
\mathcal{P}(\mathbf{w})=\int_{\Gamma}\left(-\gamma \kappa \mathbf{n}+\nabla_{\Gamma} \gamma\right) \cdot \mathbf{w}-\int_{\partial \Gamma} \gamma \boldsymbol{\nu} \cdot \mathbf{w}
$$

Onde $\boldsymbol{\nu}$ é a conormal (vide Fig. (5.2)), que é normal à linha de contato e perpendicular a normal $\mathbf{n}$. Nesta forma, é possível reconhecer as forças associadas à potência virtual, que são

- a forção superficial em $\Gamma: \mathbf{A}=-\gamma \kappa \mathbf{n}+\nabla_{\Gamma} \gamma$;

- e a força na linha de contato $\partial \Gamma($ se esta $\neq \emptyset)$ : $\mathbf{T}=-\gamma \boldsymbol{v}$.

Neste trabalho, é usada a primeira forma, $\int_{\Gamma} \gamma \nabla_{\Gamma} \cdot \mathbf{w}$, pois nela não é necessário computar a curvatura nem a força na linha de contato, além de incorporar o efeito da força de Marangoni $\left(=\nabla_{\Gamma} \gamma\right)$. 


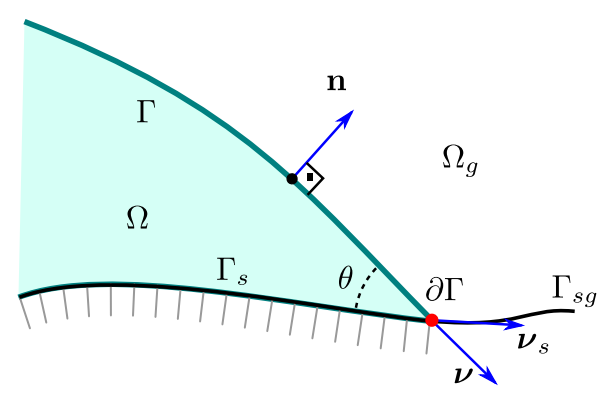

Figura 5.2: Ilustração da conormal $\boldsymbol{\nu}$ da superfície $\Gamma$, que é perpendicular à normal de $\Gamma$ e normal à linha de contato, e da conormal $\boldsymbol{\nu}_{s}$ da superfície sólida $\Gamma_{s}$, que é perpendicular à normal de $\Gamma_{s}$ de normal à linha de contato. O ângulo $\theta$ é igual ao ângulo entre $\boldsymbol{\nu}$ e $\boldsymbol{\nu}_{s}$.

\subsection{Potências virtuais na linha de contato}

O cenário agora consiste no caso quando a superfície $\Gamma$ toca no substrato sólido dividindo-o nas superfícies $\Gamma_{s}$ (interior à gota) e $\Gamma_{s g}$, como é ilustrado na Fig. (5.1), e agora as energias associadas a tais superfícies devem ser contabilizadas. Denotando por $\gamma_{s}$ e $\gamma_{s g}$ as respectivas tensões superficias, a anergia total passa a ser

$$
\mathcal{E}(\Gamma)=\int_{\Gamma} \gamma+\int_{\Gamma_{s}} \gamma_{s}+\int_{\Gamma_{s g}} \gamma_{s g}
$$

Usando a derivada de forma da energia para encontrar a potência virtual associada, obtém-se

$$
\mathcal{P}(\mathbf{w})=-\int_{\Gamma} \gamma \nabla_{\Gamma} \cdot \mathbf{w}-\int_{\partial \Gamma}\left(\gamma_{s}-\gamma_{s g}\right) \boldsymbol{\nu}_{s} \cdot \mathbf{w}
$$

onde foi usado o fato de que $\mathbf{w} \cdot \mathbf{n}=0 \mathrm{em} \Gamma_{s}$ (restrição de não penetração no sólido) e suposto que $\gamma_{s}$ e $\gamma_{s g}$ são constantes.

\subsection{Singularidade, forças dissipativas e ângulo de contato dinâmico}

\section{Condição de Navier}

Para que a linha de contato evolua, não se pode aplicar uma condição de não escorregamento em $\Gamma_{s}$. Em Dussan e Davis [20] foi observado experimentalmente que a superfície $\Gamma$ rola sobre $\Gamma_{s}$ como a esteira de um trator. Entretanto, é mostrado, por exemplo, em Shikhmurzaev [43], que todas as soluções da equação de Stokes com condição de não escorregamento leva a uma dissipação infinita na linha de contato. O 
método mais comum de se contornar o problema é relaxar a condição de não escorregamento e acrescentar à potência virtual uma lei de escorregamento do tipo Navier na superfície sólida, ou seja, acrescentar

$$
\mathcal{P}_{\Gamma, d i s}(\mathbf{w})=-\int_{\Gamma_{s}} \beta \mathbf{u} \cdot \mathbf{w}
$$

onde $\beta$ é conhecido como coeficiente de escorregamento. Uma discussão sobre a modelagem de $\beta$ pode ser encontrado em Ren [40]. Por simplicidade, $\beta$ será considerado constante, podendo ser estimado pela expressão $\beta \sim \mu / \ell$, onde $\ell$ é a espessura da camada interfacial, tipicamente dada por $\ell \simeq 1-3 \mathrm{~nm}$ (Shikhmurzaev [44], sec. 4.3.2).

\section{Ângulo de contato dinâmico}

Seja $M \doteq\left(\gamma_{s g}-\gamma_{s}\right) / \gamma$. Quando $|M| \leq 1$, é possivel definir um ângulo $\theta_{s}$ tal que

$$
\cos \theta_{s} \doteq\left(\gamma_{s g}-\gamma_{s}\right) / \gamma
$$

Substituindo-a no princípio das potências virtuais, obtém-se

$$
\int_{\Omega} \sigma: D \mathbf{w}+\int_{\Omega} \rho \frac{\mathrm{d} \mathbf{u}}{\mathrm{d} t} \cdot \mathbf{w}=\mathcal{P}_{\Gamma, d i s}(\mathbf{w})-\int_{\Gamma} \gamma \nabla \cdot \mathbf{w}+\int_{\partial \Gamma} M \boldsymbol{\nu}_{s} \cdot \mathbf{w}, \quad \forall \mathbf{w} \in W_{0} .
$$

Da arbitrariedade de w, é possível concluir que no modelo acima, o ângulo de contato $\theta$, dado por

$$
\cos \theta=\boldsymbol{\nu} \cdot \boldsymbol{\nu}_{s}
$$

é sempre igual ao ângulo estático $\theta_{s}$ em (5.23) (Buscaglia e Ausas [10]), resultado este que não corresponde nos experimentos, pois sabe-se que o ângulo varia com o tempo.

Uma forma de remediar esta situação, é adicionar um termo dissipativo na linha de contato na forma

$$
\mathcal{P}_{\partial \Gamma, d i s}(\mathbf{w})=-\int_{\partial \Gamma}\left(\zeta \mathbf{u} \cdot \boldsymbol{\nu}_{s}\right)\left(\mathbf{w} \cdot \boldsymbol{\nu}_{s}\right)
$$

Alguns modelos para $\zeta$ são estudados em Manservisi e Scardovelli [34], e têm a forma:

$$
\zeta=\frac{\gamma}{|\mathbf{u}|} R(C a)
$$

onde $C a=|\mathbf{u}| \mu / \gamma$ é o número de capilaridade e $R(C a)$ é uma potência de $C a$, baseada em curvas de dados experimentais. 


\subsection{Formulação variacional}

Incorporando no princípio das potências virtuais (5.1) a restrição fraca de incompressibilidade $(\nabla \cdot \mathbf{u}, q)=0, \forall q \in Q \cup L^{2}(\Omega)$ e passando as derivadas temporais para a formulação ALE, é obtida uma formulação variacional análoga à apresentada no primeiro capítulo, reescrita aqui por conveniência: encontrar $(\mathbf{u}, p) \in W_{u} \times Q$ tal que

$$
\begin{aligned}
& \left(\rho \partial_{t}^{*} \mathbf{u}, \mathbf{w}\right)+a(\mathbf{u}, \mathbf{w})+c(\mathbf{c} ; \mathbf{u}, \mathbf{w})-b(p, \mathbf{w})=\mathcal{P}(\mathbf{w})+\mathcal{P}_{\Gamma, d i s}(\mathbf{w})+\mathcal{P}_{\partial \Gamma, d i s}(\mathbf{w}) \\
& -b(q, \mathbf{u})=0
\end{aligned}
$$

$\forall(\mathbf{w}, q) \in W_{0} \times Q$. Supondo que as velocidades estejam em $\left(H^{1}(\Omega)\right)^{d}$, definem-se

$$
\begin{aligned}
& W_{0} \doteq\left\{\mathbf{w} \in\left(H^{1}(\Omega)\right)^{d} \mid \mathbf{w} \cdot \mathbf{n}=0 \mathrm{em} \Gamma_{s}\right\}, \\
& W_{u} \doteq\left\{\mathbf{w} \in\left(H^{1}(\Omega)\right)^{d} \mid \mathbf{w} \cdot \mathbf{n}=U_{n} \mathrm{em} \Gamma_{s}\right\},
\end{aligned}
$$

onde $U_{n}$ é a componente normal da velocidade do sólido. As restrições em $\Gamma_{s}$ vêm do fato de que o substrato sólido é impermeável.

\subsection{Dificuldades e outros modelos}

Deve ser observado que os termos na linha de contato, que são da forma $\int_{\partial \Gamma} \mathbf{r} \cdot \mathbf{w}$ para algum campo r, não são limitados em $\left(H^{1}(\Omega)\right)^{d}$, o que pode levar a consequências numéricas, apesar de que, sob discretização, estes termos são bem definidos, uma vez que o espaço discreto da velocidade consiste em funções contínuas (Buscaglia e Ausas [10]).

Para a simulação reproduzir fielmente as observações experimentais, acredita-se que efeitos microscópicos devam ser incorporados à descrição hidrodinâmica para regularizar a deficiência matemática na linha de contato. Em Bonn et al. [4], são citados mecanismos propostos para tratar esta singularidade, entre eles, camada precursora, filme molecular, condição de Navier, interface difusa, entre outros. Existe também o modelo de Shikhmurzaev [44], onde as superfícies são consideradas uma fase, e atribuídas à elas uma densidade superficial $\rho^{s}$ e uma velocidade superficial $\mathbf{u}^{s}$, onde $\mathbf{u}^{s} \neq \mathbf{u}$. Neste modelo, são consideradas perturbações dos valores de equilíbrio da tensão superficial (que é uma incógnita) e obtidas equações para a densidade superficial $\rho^{s}$, parecidas com equações de concentração de surfactantes. Um resumo de seu modelo pode ser encontrado em Sprittles e Shikhmurzaev [47]. 
O modelo apresentado até aqui, não inclui fenômenos importantes como histerese, que podem depender da heterogeneidade da superfície sólida, como sua rugosidade e orientação de moléculas. 



\section{Capítulo 6}

\section{Aspectos computacionais e exemplos numéricos}

\subsection{Discretização e métodos}

\subsubsection{Formulação variacional discreta final}

O problema da discretização espacial e temporal da formulação variacional correspondente ao cenário de uma gota se espalhando em um superfície sólida levando em conta as forças capilares e as forças dissipativas, fica: encontrar $\left(\mathbf{u}_{h}^{n+1}, p_{h}^{n+1}\right) \in W_{u, h} \times Q_{h}$ tal que

$$
\begin{gathered}
\int_{\tilde{\Omega}^{n+1 / 2}}\left[\rho\left(\delta_{t} \mathbf{u}_{h}^{n}+\nabla \mathbf{u}_{h}^{n+1 / 2} \cdot \mathbf{c}_{h}^{n+1 / 2}\right) \cdot \mathbf{w}_{h}+\left(-\mathbf{I}_{d} p_{h}^{n+1}+2 \mu D \mathbf{u}_{h}^{n+1 / 2}\right): D \mathbf{w}_{h}\right]+ \\
+\int_{\tilde{\Gamma}^{n+1 / 2}} \beta \mathbf{u}_{h}^{n+1 / 2} \cdot \mathbf{w}_{h}=-\int_{\tilde{\Gamma}^{n+1 / 2}} \gamma \nabla_{\Gamma} \cdot \mathbf{w}_{h}+\int_{\partial \tilde{\Gamma}^{n+1 / 2}} \cos \theta_{s} \boldsymbol{\nu}_{s} \cdot \mathbf{w}_{h}- \\
\quad-\int_{\partial \tilde{\Gamma}^{n+1 / 2}}\left(\zeta \mathbf{u}_{h}^{n+1 / 2} \cdot \boldsymbol{\nu}_{s}\right)\left(\mathbf{w}_{h} \cdot \boldsymbol{\nu}_{s}\right), \\
-\int_{\tilde{\Omega}^{n+1 / 2}} q_{h} \nabla \cdot \mathbf{u}_{h}^{n+1 / 2}=0,
\end{gathered}
$$



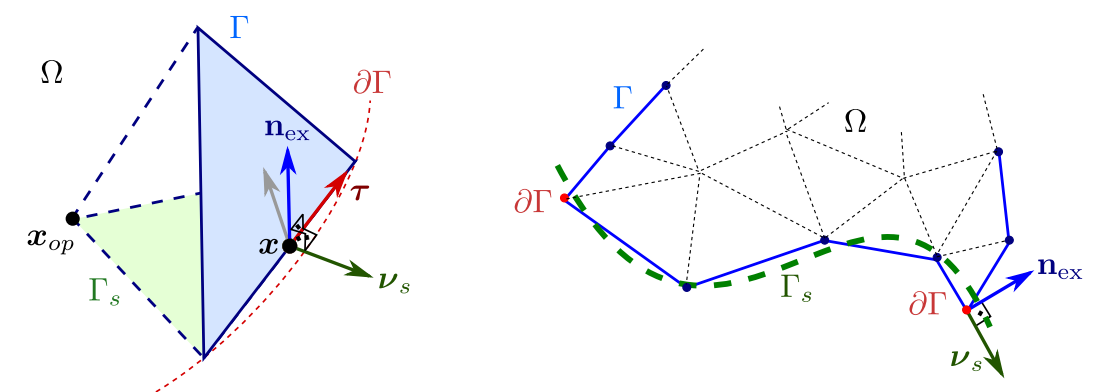

Figura 6.1: Conormal de $\Gamma_{s}$, denotada por $\boldsymbol{\nu}_{s}$, em 3D (à esquerda) e 2D (à direita).

para todo $\left(\mathbf{w}_{h}, q_{h}\right) \in W_{0, h} \times Q_{h}$, com uma condição inicial adequada, onde

$$
\begin{aligned}
& W_{0, h} \doteq\left\{\mathbf{w} \in\left(H^{1}(\Omega)\right)^{d} \mid \mathbf{w} \cdot \mathbf{n}=0 \text { em } \Gamma_{s}\right\}, \\
& W_{u, h} \doteq\left\{\mathbf{w} \in\left(H^{1}(\Omega)\right)^{d} \mid \mathbf{w} \cdot \mathbf{n}=U_{n} \text { em } \Gamma_{s}\right\},
\end{aligned}
$$

onde, relembrando, $\tilde{\Omega}^{n+1 / 2}, \tilde{\Gamma}^{n+1 / 2}$ e $\partial \tilde{\Gamma}^{n+1 / 2}$ são aproximações de $\Omega^{n+1 / 2}, \Gamma^{n+1 / 2}$ e $\partial \Gamma^{n+1 / 2}$, respectivamente, dadas pelo avanço explícito da malha $\mathbf{x}_{h}$ com Adams-Bashforth (ver Alg. (2.1)).

\subsubsection{Cálculos nas superfícies e linha de contato.}

Os termos em $\int_{\tilde{\Gamma}^{n+1 / 2}}(\ldots)$ e $\int_{\partial \tilde{\Gamma}^{n+1 / 2}}(\ldots)$, que envolvem um gradiente de superfície e mapeamentos para elementos unitários, são computados com as ferramentas introduzidas na Sec. (4.5). Os coeficientes $\beta, \zeta$ e $\theta_{s}$ são dados e considerados constantes por simplicidade.

Em 3D, os versores $\boldsymbol{\nu}_{s}$ presentes nas integrais em $\partial \tilde{\Gamma}^{n+1 / 2}$ são computados, em cada ponto de quadratura $\boldsymbol{x}$, como

$$
\boldsymbol{\nu}_{s}(\boldsymbol{x})=\frac{\boldsymbol{\tau}(\boldsymbol{x}) \times \mathbf{n}_{\mathrm{ex}}(\boldsymbol{x})}{\left\|\boldsymbol{\tau}(\boldsymbol{x}) \times \mathbf{n}_{\mathrm{ex}}(\boldsymbol{x})\right\|},
$$

onde $\boldsymbol{\tau}(\boldsymbol{x})$ é o vetor tangente à linha de contato e $\mathbf{n}_{\mathrm{ex}}(\boldsymbol{x})$ é a normal, como ilustra a figura esquerda da Fig. (6.1). No programa implementado, $\mathbf{n}_{\text {ex }}$ é um campo vetorial unitário (dado pelo usuário) definido em todo espaço $\mathbb{R}^{d}$, e, idealmente, é igual a extensão normal da normal exata da superfície sólida exata $\Gamma_{s}^{\text {ex }}$, que é conhecida. A tangente é dada

$$
\boldsymbol{\tau}=\nabla_{s} \boldsymbol{\Phi}(s) /\left\|\nabla_{s} \boldsymbol{\Phi}(s)\right\|
$$

onde $\boldsymbol{\Phi}(s), s \in \mathbb{R}$, é a transformação que leva $[-1,1] \in \mathbb{R}$ na aresta na qual se está 
integrando. No caso de elementos lineares, pode-se calcular $\boldsymbol{\tau}$ mais facilmente com os vértices da aresta, entretanto, a expressão anterior é conveniente para generalizar aos casos com arestas curvas. No programa implementado, a direção de $\boldsymbol{\tau}$ depende da orientação do tetraedro no qual está contido, por isso, o sinal de $\boldsymbol{\nu}_{s}$ é consertado usando o vetor $\left(\boldsymbol{x}-\boldsymbol{x}_{o p}\right)$ (ver Fig. (6.1)), da seguinte forma: se $\boldsymbol{\nu}_{s} \cdot\left(\boldsymbol{x}-\boldsymbol{x}_{o p}\right)<0$, $\nu_{s} \leftarrow-\nu_{s}$.

\subsection{Exemplos numéricos}

\subsubsection{Superfície livre}

Num primeiro problema trata-se de um fluido sob efeito da gravidade confinado em um recipiente retangular. Este teste é conhecido na literatura como sloshing. Os parâmetros escolhidos foram

- profundidade do fluido não perturbado $h=1.5$;

- largura do recipiente $d=1.0$;

- amplitude da perturbação inicial $a_{0}=0.01$

- viscosidade e densidade $\mu=0.01$ e $\rho=1$, respectivamente;

- Tensão superficial $\gamma=0$;

- dissipações nulas na linha de contato, i.e., $\beta=\zeta=0$.

Para pequenas oscilações (Perot e Nallapati [38]), o período da oscilação é dado por:

$$
T=\left[\frac{g}{4 \pi L} \tanh \left(\frac{\pi D}{L}\right)\right]^{-1 / 2}
$$

Foi usada uma malha com 636 elementos. O erro no período foi calculado com a expressão:

$$
\left|\omega_{\text {exato }}-\omega_{h}\right| / \omega_{\text {exato }}
$$

onde $\omega=2 \pi / T$. O erro obtido foi de $\sim 8 \%$. As pressões e o campo de velocidade podem ser vistos na Fig. (6.2). 

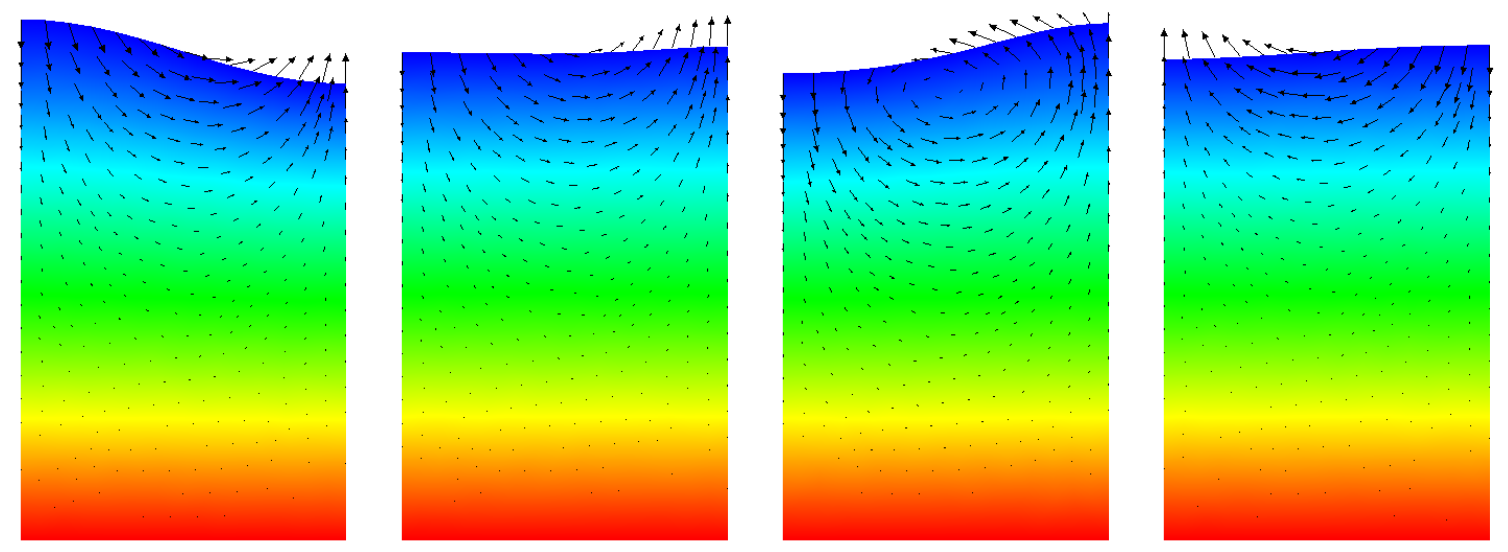

Figura 6.2: Sloshing: campos de pressão e velocidade em vários instantes. A pressão vermelha indica a pressão mais alta.

\subsubsection{Gota estática}

Este exemplo consiste em estudar a convergência do campo de pressão em uma gota estática de raio 1, a fim validar o termo da força superficial. A curvatura média da gota é constante em toda a superfície, e dada por $\kappa=(d-1) / R$, onde $d$ é a dimensão espacial e $R=1$ o raio da gota. A tensão superficial é escolhida como $\gamma=1$, assim como a densidade $\rho$, e a viscosidade foi tomada como $\mu=10^{-3}$. Foi eliminado o termo convectivo do problema e este foi rodado em 1 passo de tempo. A solução obtida foi comparada com a solução exata da pressão, que é dada por $p=\gamma \kappa$. Foram testados 3 elementos, e também foram testadas versões melhoradas do cálculo correspondente a tensão superficial, i.e, do cálculo de $\int_{\Gamma} \gamma \nabla_{\Gamma} \cdot \mathbf{w}_{h}$.

A versão melhorada é obtida de uma adaptação feita no método proposto em Sven Groß [49] para level-set. Consiste e trocar o termo $\int_{\Gamma} \gamma \nabla_{\Gamma} \cdot \mathbf{w}_{h}$ por $\int_{\Gamma} \gamma \tilde{\mathbf{P}}: \nabla_{\Gamma} \mathbf{w}_{h}$, onde $\tilde{\mathbf{P}}=\mathbf{I}_{d}-\tilde{\mathbf{n}} \otimes \tilde{\mathbf{n}}$, onde $\tilde{\mathbf{n}}$ é uma normal diferente do da malha $\mathbf{n}_{h}$ que é calculada em cada triângulo. As normais que serão testadas aqui, são $\mathbf{n}_{\text {esf }}$ e $\mathbf{n}_{\text {cons }}$, onde $\mathbf{n}_{\text {cons }}$ é a interpolação da normais nos nós encontradas com o método consistente com a massa introduzida em (2.5.3), enquanto que $\mathbf{n}_{\text {esf }}$ é a interpolação das normais nos nós encontradas com o método de Max.

Os gráficos com os erros de acordo com o grau de refinamento da malha podem ser vistos nas Figs. (6.3) (caso 2D) e (6.4) (caso 3D). Em 2D, evitou-se que os nós ficam equidistribuidos sobre a superfície para evitar super convergência. Pode-se notar uma significativa redução do erro na pressão quando usada a versão melhorada com $\mathbf{n}_{\text {esf }}$, tanto em 2D quanto em 3D, além de um ganho na ordem de convergência. Entretanto, sabe-se que $\mathbf{n}_{\text {esf }}$ é a normal exata de uma esfera quando a malha tem seus nós sobre a 


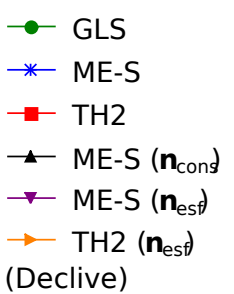

(a) Legenda.

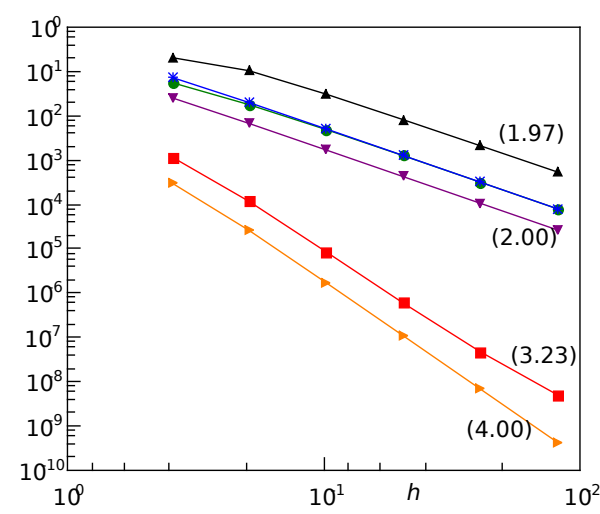

(c) $\left\|p-p_{h}\right\|_{L^{2}}$.

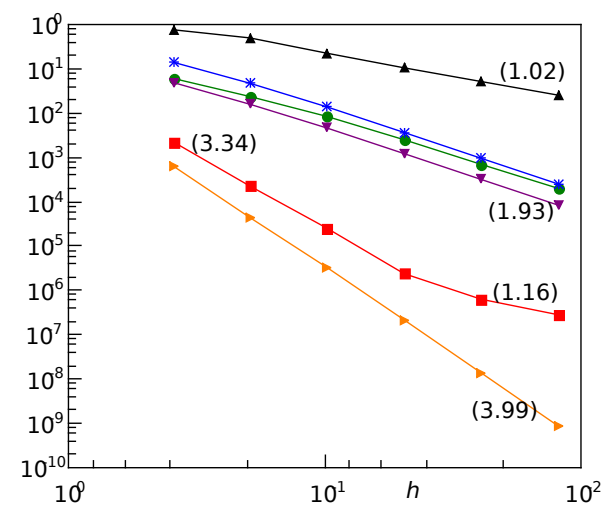

(e) $\left\|p-p_{h}\right\|_{\infty}$.

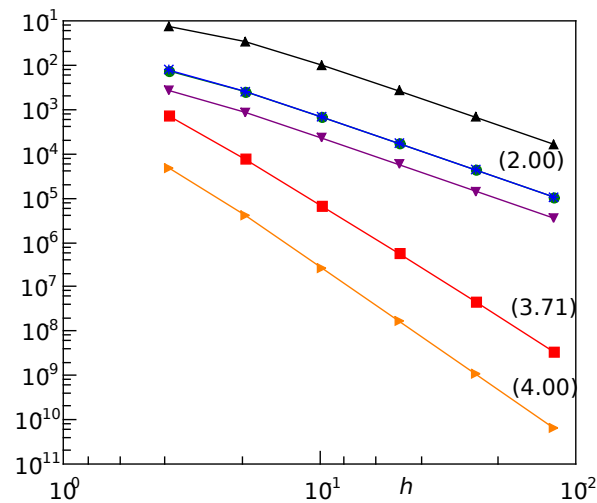

(b) $\left\|\mathbf{u}-\mathbf{u}_{h}\right\|_{L^{2}}$.

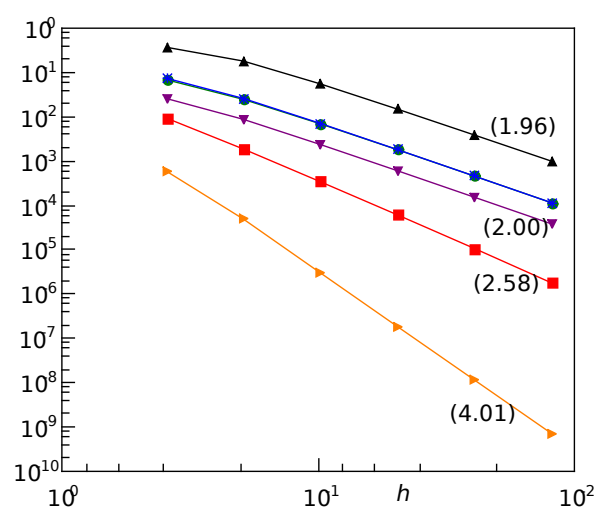

(d) $\left\|\nabla \mathbf{u}-\nabla \mathbf{u}_{h}\right\|_{L^{2}}$.

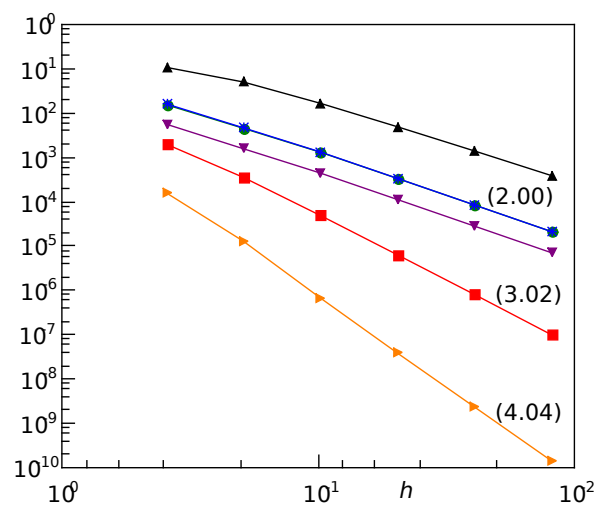

(f) $\left\|\mathbf{u}-\mathbf{u}_{h}\right\|_{\infty}$.

Figura 6.3: Erros em $L^{2}$ da velocidade e pressão para vários elementos em 2D.

esfera, o que é o caso. Seria necessário testar outras superfícies em que $\mathbf{n}_{\text {esf }}$ não dê a normal exata. 


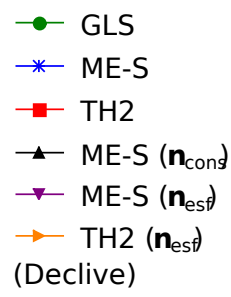

(a) Legenda.

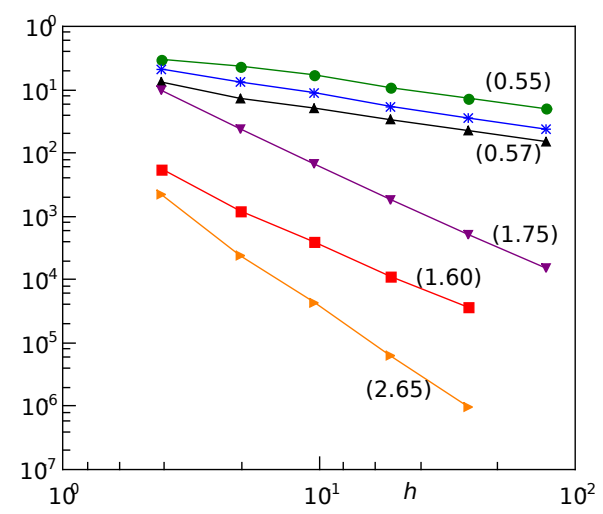

(c) $\left\|p-p_{h}\right\|_{L^{2}}$.

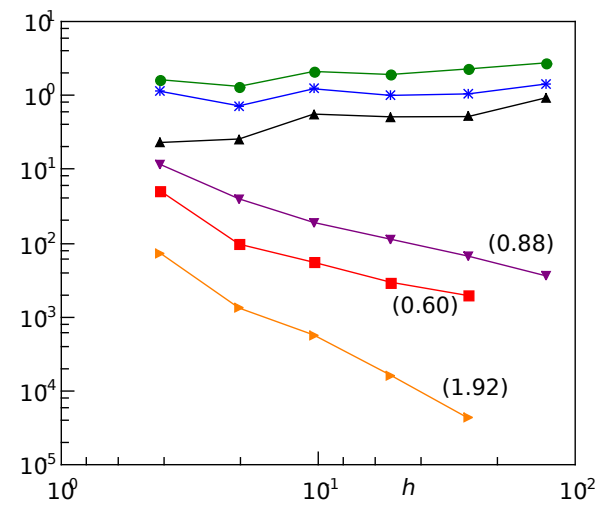

(e) $\left\|p-p_{h}\right\|_{\infty}$.

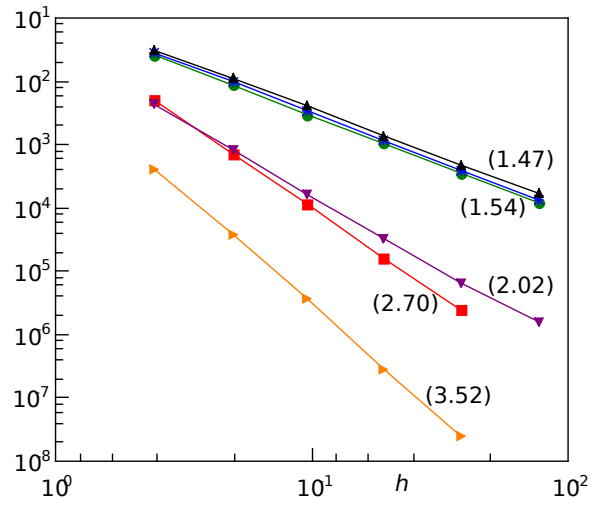

(b) $\left\|\mathbf{u}-\mathbf{u}_{h}\right\|_{L^{2}}$.

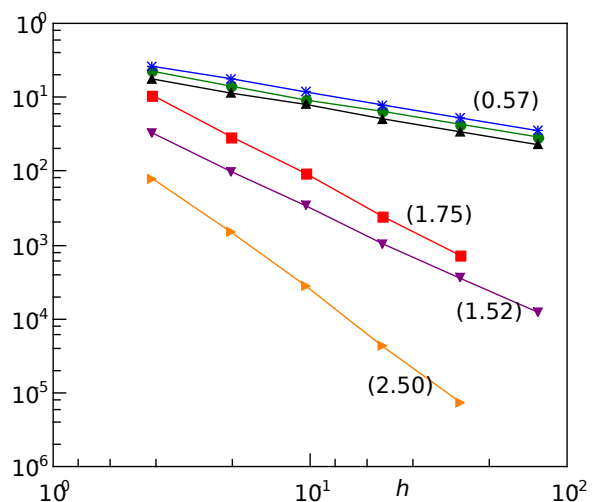

(d) $\left\|\nabla \mathbf{u}-\nabla \mathbf{u}_{h}\right\|_{L^{2}}$.

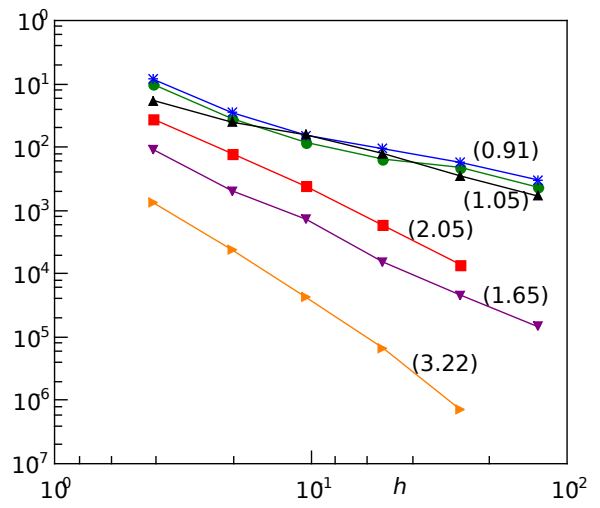

(f) $\left\|\mathbf{u}-\mathbf{u}_{h}\right\|_{\infty}$.

Figura 6.4: Erros em $L^{2}$ da velocidade e pressão para vários elementos em 3D. 

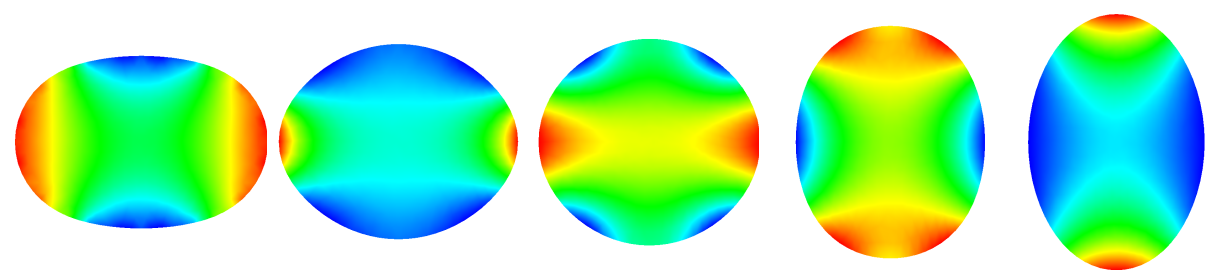

Figura 6.5: Oscilação de uma gota inicialmente perturbada elipticamente. As cores indicam a pressão.

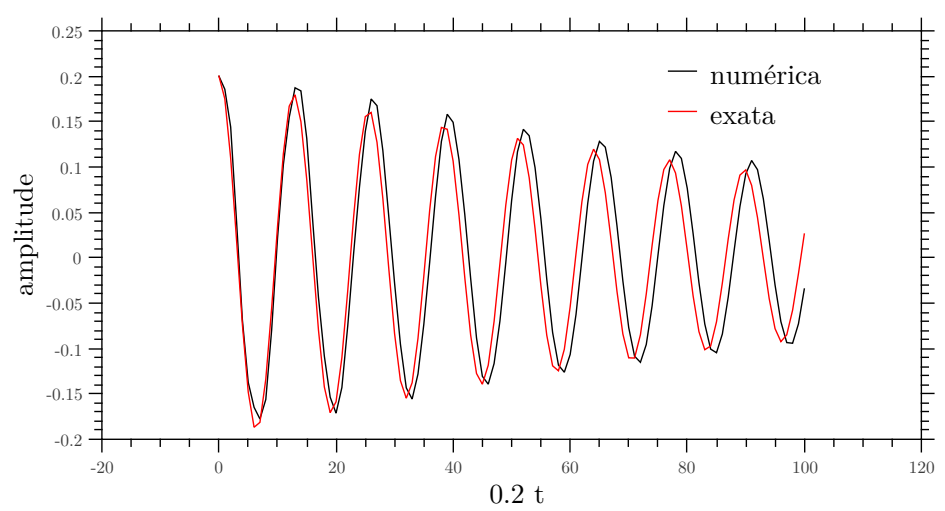

Figura 6.6: Comparação da amplitude da gota com a solução exata.

\subsubsection{Gota oscilante}

Este problema trata de uma gota inicialmente perturbada elipticamente que oscila até o equilíbrio. Sua frequência, para pequenas oscilações, é dada por Brant Foote [5]:

$$
\omega_{n}=\frac{\left(n^{3}-n\right) \gamma}{\left(\rho_{L}+\rho_{G}\right) R^{3}}
$$

A amplitude $A(t)$ de oscilação decresce com a lei

$$
A(t)=A_{0} \exp (-t / \tau)
$$

onde

$$
\tau=\mu \frac{R^{2}}{4}
$$

e $A_{0}$ é a amplitude inicial. São escolhidos $R=1, \rho=1, A_{0}=0.2, \mu=0.01$ e $\gamma=1$. A comparação da solução obtida com a exata pode ser vista no gráfico (6.6), enquanto o perfil da gota em vários instantes de tempo pode ser visto na figura (6.5). 


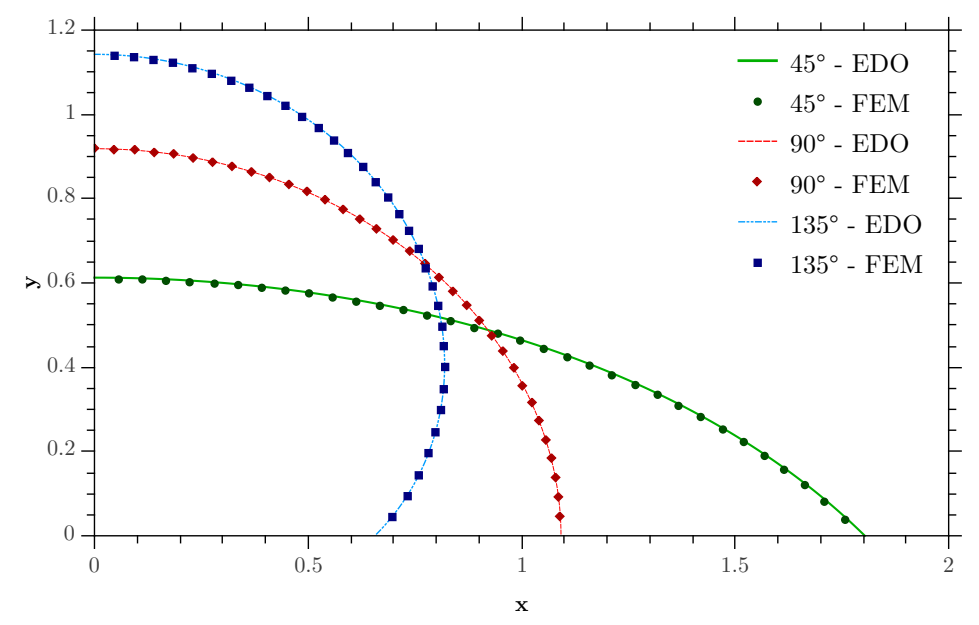

Figura 6.7: Validação do ângulo estático comparando a solução obtida com a solução de uma EDO.

\subsection{4 Ângulo estático}

Este problema valida a imposição do ângulo estático por meio do termo $\int_{\partial \Gamma} \cos \theta_{s} \boldsymbol{\nu}_{s} \cdot \mathbf{w}_{h}$ em uma gota inicialmente com $90^{\circ}$. São testados os ângulos $45^{\circ}, 90^{\circ}$ e $135^{\circ}$. O programa é rodado até que a gota chegue em seu estado de repouso, e seu perfil final é comparado com o perfil da solução de uma EDO dada em Pozrikidis [39]. Nesta referência, é possível encontrar um programa em Matlab que resolver tal EDO. A EDO foi resolvida com método de Runge-Kutta de quarta ordem, com passo de integração $\delta s=0.0001$. O problema foi resolvido com o elemento TH2, com $\gamma=1.5, \mu=0.5, \rho=1$ e $g=-1$ (aceleração da gravidade). A malha possui 454 elementos e 965 nós. O resultado pode ser visto na Fig. (6.7).

\subsection{5 Ângulo dinâmico 2D}

Neste teste é verificado o quanto a dissipação na linha de contato interfere no desvio do ângulo de contato do ângulo estático. A configuração inicial é uma gota de raio estático 1 depositada em um sólido, como ilustra a gota azul na Fig. (6.9). O gráfico do ângulo de contato pelo tempo foi feito para dois valores de coeficiente dissipação $\zeta$, e podem ser vistos na Fig. (6.8).

$$
v=10^{-5}, \quad \gamma=0.075, \quad \beta=10^{-5}, \quad \Delta t=2 \cdot 10^{-7} .
$$




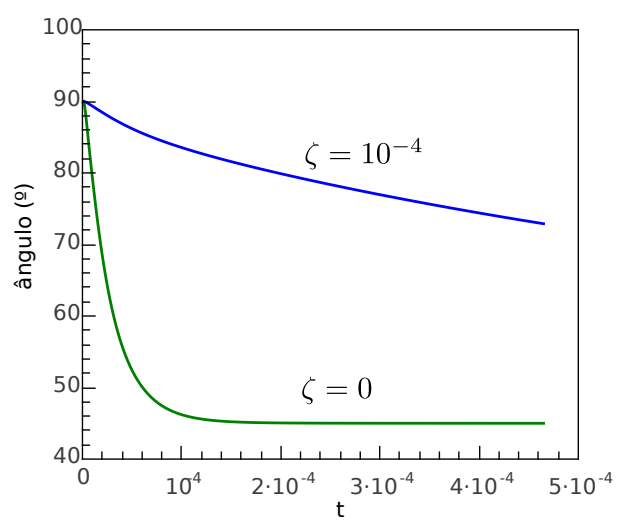

Figura 6.8: Ângulo de contato dinâmico com dissipação $\beta=10^{-5}$ na superfície sólidolíquido, para dois valores diferentes de dissipação na linha de contato.
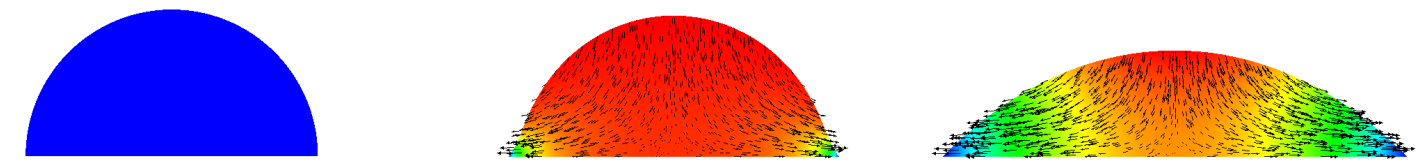

Figura 6.9: Dinâmica do ângulo de contato, em vários instantes diferentes. A cores indicam os valores da pressão.

\subsubsection{Outros testes}

Dois outros testes apenas para demonstração foram feitos. O primeiro, é uma gota em 3D inicialmente depositada em um substrato sólido, com ângulo de contato de $90^{\circ}$. Lentamente, a gota se contrai para o ângulo estático imposto $\left(135^{\circ}\right)$, como é visto na Fig. (6.10).

O segundo teste é de uma gota pendente. Uma gota está inicialmente em um teto, e a ação da gravidade puxa a gota para baixo, modelando alguns perfis para gota que podem ser vistos nas Fig. (6.11) e Fig. (6.12). fffff 
6. Aspectos COMPUtacionais E EXEMPlos nUmÉRICOS
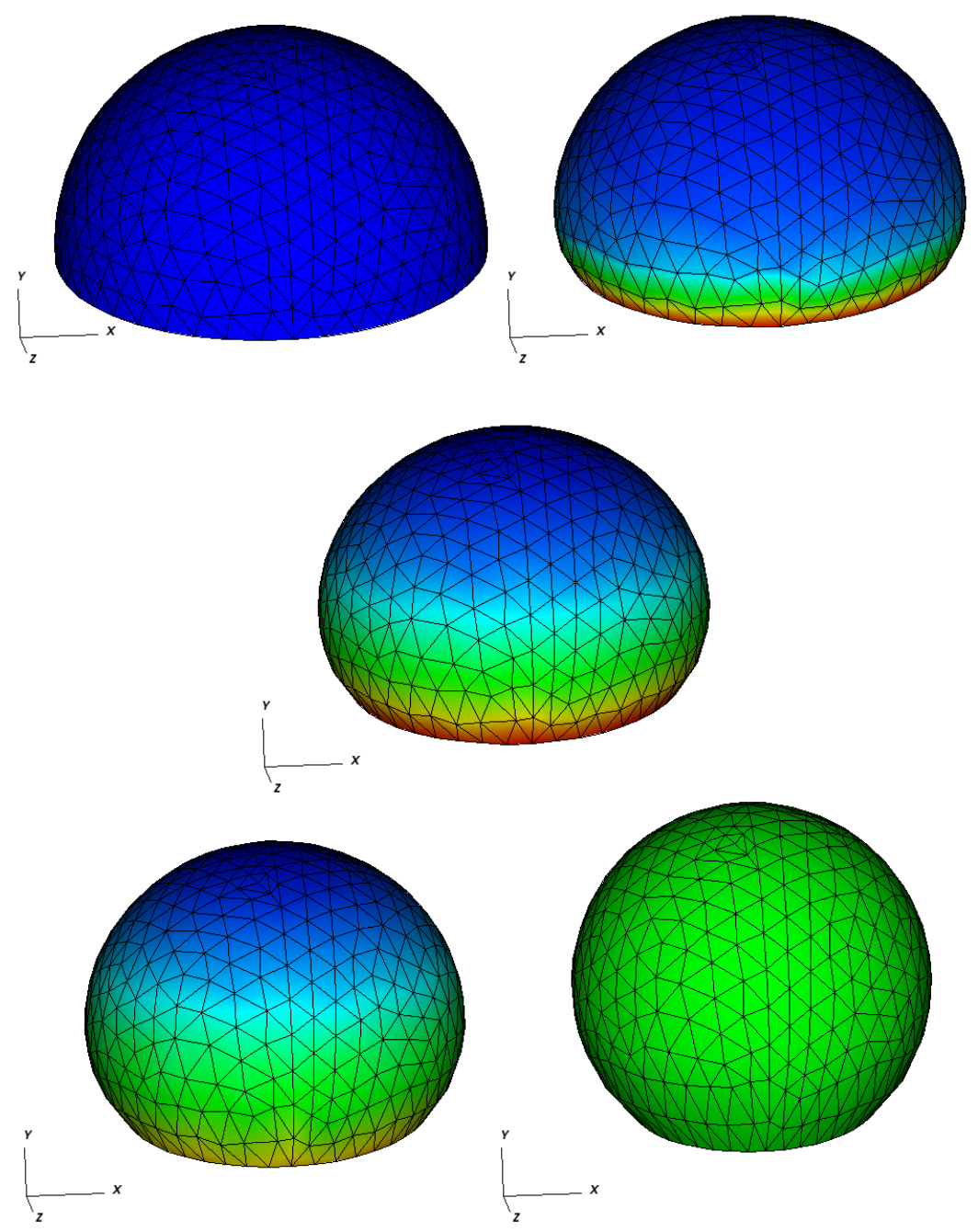

Figura 6.10: Ângulo de contato dinâmico de uma gota 3D, com ângulo estático de $135^{\circ}$.

84 


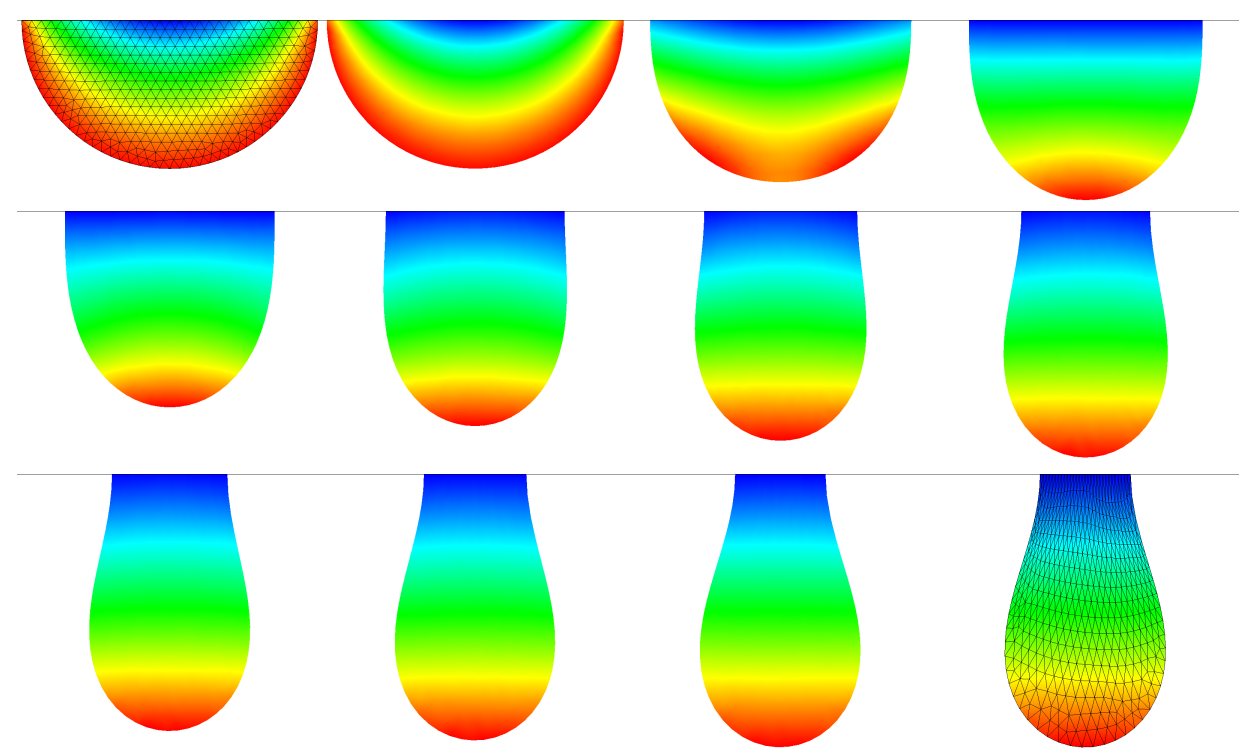

Figura 6.11: Gota pendente bidimensional em vários passos de tempo, com ângulo estático de $90^{\circ}$. As cores indicam a pressão.

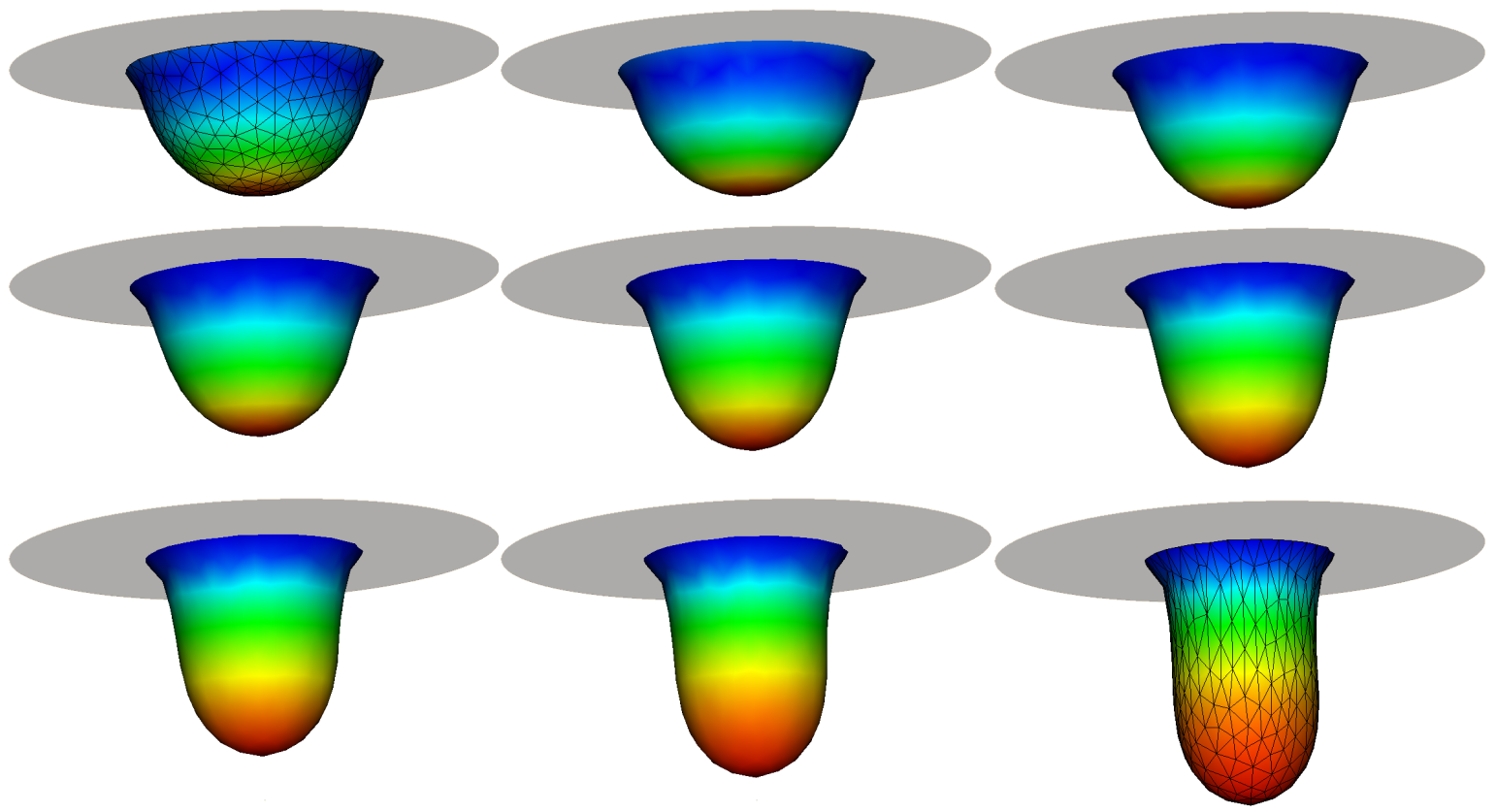

Figura 6.12: Gota pendente tridimensional em vários passos de tempo, com ângulo estático de $45^{\circ}$. As cores indicam a magnitude da velocidade que está alinhada à gravidade. 



\section{Capítulo 7}

\section{Conclusão}

No primeiro capítulo foi investigada com detalhes a formulação ALE para as equações de Navier-Stokes. Estudos numéricos comparando a precisão dos elementos $\left(\mathrm{P}_{1} \mathrm{P}_{1}\right.$ (GLS), mini-elemento simplificado, Crouzeix-Raviart simplificado, e Taylor-Hood) foram feitos, e, apesar de não haver nenhuma surpresa com relação à convergência espacial, algumas informações úteis não tão divulgadas foram apresentadas, como por exemplo, a aproximação do elemento Crouzeix-Raviart (CR) apresentar um erro maior para a pressão em certos casos do que o elemento de TH2 (fato explicado em Chamberland et al. [12]), e, ainda com o elemento CR, é melhor como base do espaço descontínuo da pressão o polinômio $P_{1}(K)$ do que o $P_{1}(\hat{K})$. Mostrou-se também que utilizando o método de Crank-Nicolson com o método ALE na forma não conservativa, obtém-se convergência quadrática no tempo (pelo menos com os testes realizados), sem preocupações com a GCL.

Ainda no primeiro capítulo, foi apresentado um método de como avançar com a malha sem acoplar suas posições nodais com o sistema de equações (via AdamsBashforth), que converge com $\Delta t^{2}$. Como trabalho futuro, é interessante investigar que condições de estabilidade este método explícito acarreta, comparando resultados com os métodos monolíticos. Foi utilizado no trabalho malhas com elementos curvos, que, como mostraram os testes, apresentam resultados mais precisos. Entretanto, em simulações que envolvem grandes deformações, onde operações topológicas sobre a malha são imprescindíveis, trabalhar com elementos curvos pode levar a difíceis complicações, como a interpolação nos nós de alta ordem. 
Finalmente, foram apresentados alguns resultados sobre simulações da dinâmica de molhamento. Foram feitos testes que validam a teoria e o funcionamento de cada componente do programa elaborado. Alguns testes com o cálculo da força superficial $\int_{\Gamma} \gamma \nabla_{\Gamma} \cdot \mathbf{w}$ mostraram bons resultados com a versão modificada, entretanto, testes com gostas estáticas são muitos restritos. Futuramente, a modificação será investigada em superfícies genéricas. As ferramentas computacionais desenvolvidas abrem possibilidade para vários tipos de simulações de espalhamento com geométricas arbitrárias, que serão exploradas em estudos mais complexos envolvendo fenômenos de superfície. 


\section{Referências Bibliográficas}

[1] Ainsworth, M. (2001). Essential boundary conditions and multi-point constraints in finite element analysis. Computer methods in applied mechanics and engineering, 190(48):6323-6339.

[2] Baltussen, M., Choi, Y., e Hulsen, M. (2011). Weakly-imposed Dirichlet boundary conditions for non-Newtonian fluid flow. of Non-Newtonian Fluid, 166(17-18):9931003.

[3] Bonito, A. e Nochetto, R. (2010). Geometrically consistent mesh modification. submitted for publication, páginas 1-23.

[4] Bonn, D., Eggers, J., Indekeu, J., Meunier, J., e Rolley, E. (2009). Wetting and spreading. Reviews of Modern Physics, 81(2):739-805.

[5] Brant Foote, G. (1973). A numerical method for studying liquid drop behavior: Simple oscillation. Journal of Computational Physics, 11(4):507-530.

[6] Brenner, S. C. e Scott, L. R. (2008). The Mathematical Theory of Finite Element Methods. Springer; 3rd edition.

[7] Brezzi, F., Demkowicz, L. F., Durán, R. G., Falk, R. S., Fortin, M., e Boffi, D. (2006). Mixed Finite Elements, Compatibility Conditions, and Applications: Lectures given at the C.I.M.E. Springer.

[8] Brezzi, F. e Fortin, M. (1991). Mixed and Hybrid Finite Element Methods. Springer.

[9] Bruus, H. (2007). Theoretical Microfluidics. Oxford University Press. 
[10] Buscaglia, G. C. e Ausas, R. F. (2011). Variational formulations for surface tension, capillarity and wetting. Computer Methods in Applied Mechanics and Engineering, 200(45-46):3011-3025.

[11] Cardoze, D., Cunha, A., Miller, G., Phillips, T., e Walkington, N. (2004). A bezierbased approach to unstructured moving meshes. Em Proceedings of the twentieth annual symposium on Computational geometry, páginas 310-319. ACM.

[12] Chamberland, E., Fortin, a., e Fortin, M. (2010). Comparison of the performance of some finite element discretizations for large deformation elasticity problems. Computers \& Structures, 88(11-12):664-673.

[13] Codina, R. (2001). A stabilized finite element method for generalized stationary incompressible flows. Computer Methods in Applied Mechanics and, 190:2681-2706.

[14] Codina, R., Blasco, J., Buscaglia, G. C., e Huerta, A. (2001). Implementation of a stabilized finite element formulation for the incompressible Navier-Stokes equations based on a pressure gradient projection. International Journal for Numerical Methods in Fluids, 37(4):419-444.

[15] Crouzeix, M. e Raviart, P. A. (1973). Conforming and nonconforming finite element methods for solving the stationary Stokes equations I. RAIRO Anal Numer, 7:33-76.

[16] Cuvelier, C., Segal, A., e Steenhoven, A. A. (1986). Finite element methods and Navier-Stokes equations. Springer.

[17] de Gennes, P.-G., Brochard-Wyart, F., e Quéré, D. (2004). Capillarity and Wetting Phenomena. Drops, Bubbles, Pearls, Waves. Springer.

[18] Delfour, M. C. e Zolésio, J.-P. (2010). Shapes and Geometries: Metrics, Analysis, Differential Calculus, and Optimization. SIAM-Sociecty for Industrial and Applied Mathematics, 2 edição.

[19] Donea, J., Huerta, A., Ponthot, J., e Rodiguez-Ferran, A. (2004). Arbitrary Lagrangian-Eulerian Methods. Encyclopedia of Computational Mechanics, E. Stein, páginas 1-25.

[20] Dussan, E. B. e Davis, S. H. (1974). On the motion of a fluid-fluid interface along a solid surface. Journal of Fluid Mechanics, 65:71-95. 
[21] Engelman, M., Sani, R., e Gresho, P. (1982). The implementation of normal and/or tangential boundary conditions in finite element codes for incompressible fluid flow. International Journal for Numerical Methods in Fluids, 2(3):225-238.

[22] Étienne, S., Garon, a., e Pelletier, D. (2009). Perspective on the geometric conservation law and finite element methods for ALE simulations of incompressible flow. Journal of Computational Physics, 228(7):2313-2333.

[23] Formaggia, L. (2004). Stability analysis of second-order time accurate schemes for ALE-FEM. Computer Methods in Applied Mechanics and Engineering, 193(3941):4097-4116.

[24] Formaggia, L. e Nobile, F. (1999). A stability analysis for the arbitrary Lagrangian Eulerian formulation with finite elements. east west journal of numerical mathematics, 7:105-132.

[25] Förster, C., Wall, W. a., e Ramm, E. (2006). On the geometric conservation law in transient flow calculations on deforming domains. International Journal for Numerical Methods in Fluids, 50(12):1369-1379.

[26] Gerstenberger, A. e Wall, W. A. (2010). An embedded Dirichlet formulation for 3D continua. Online, (November 2009):537-563.

[27] Gresho, P. M. e Sani, R. L. (2000). Incompressible Flow and the Finite Element Method, volume 2. Wiley.

[28] Guillard, H. e Farhat, C. (2000). On the significance of the geometric conservation law for flow computations on moving meshes. Computer Methods in Applied Mechanics and Engineering, 190(11-12):1467-1482.

[29] Gurtin, M. E., Fried, E., e Anand, L. (2010). The Mechanics and Thermodynamics of Continua. Cambridge University Press.

[30] Hautefeuille, M., Annavarapu, C., e Dolbow, J. (2011). Robust imposition of Dirichlet boundary conditions on embedded surfaces. International Journal for $\mathrm{Nu}$ merical Methods in Engineering, (September 2010):1-6.

[31] Hughes, T. e Franca, L. (1989). A new finite element formulation for computational fluid dynamics: VIII. The Galerkin/least-squares method for advective-diffusive equations. Computer Methods in Applied, 73(2):173-189. 
[32] Klingner, B. (2008). Tetrahedral Mesh Improvement. Tese de doutorado, University of California.

[33] Kovasznay, L. (1948). Laminar flow behind a two-dimensional grid. Proc. Camb. Philos. Soc, página 1.

[34] Manservisi, S. e Scardovelli, R. (2009). A variational approach to the contact angle dynamics of spreading droplets. Computers \&s Fluids, 38(2):406-424.

[35] Max, N. (1999). Weights for Computing Vertex Normals from Facet Normals. Journal of graphics, gpu, and game tools, 4:1-6.

[36] Nobile, F. (2001). Numerical approximation of fluid-structure interaction problems with application to haemodynamics. Tese de doutorado, Ecole Polytechnique Féderale de Lausanne.

[37] Pauletti, M. (2008). Parametric AFEM for geometric evolution equation and coupled fluid-membrane interaction. Tese de doutorado.

[38] Perot, B. e Nallapati, R. (2003). A moving unstructured staggered mesh method for the simulation of incompressible free-surface flows. Journal of Computational Physics, 184(1):192-214.

[39] Pozrikidis, C. (2009). Fluid Dynamics: Theory, Computation, and Numerical Simulation. Springer, 2 edição.

[40] Ren, W. (2006). Boundary Conditions for the Moving Contact Line Problem. New York.

[41] Scovazzi, G. e Hughes, T. J. (2007). Lecture Notes on Continuum Mechanics on Arbitrary Moving Domains.

[42] Shewchuk, J. (2002). What Is a Good Linear Finite Element? Interpolation, Conditioning, Anisotropy, and Quality Measures. Em Eleventh International Meshing Roundtable, páginas 115-126.

[43] Shikhmurzaev, Y. (2006). Singularities at the moving contact line. Mathematical, physical and computational aspects. Physica D: Nonlinear Phenomena, 217(2):121133.

[44] Shikhmurzaev, Y. D. (2007). Capillary Flows with Forming Interfaces. Chapman and Hall/CRC; 1 edition. 
[45] Soulaimani, A., Fortin, M., Ouellet, Y., Dhatt, G., e Bertrand, F. (1987). Simple continuous pressure elements for two-and three-dimensional incompressible flows. Computer methods in applied mechanics and engineering, 62(1):47-69.

[46] Sousa, F. S. (2006). Simulação de escoamentos multifásicos em malhas não estruturadas. Tese de doutorado, Universidade de São Paulo.

[47] Sprittles, J. E. e Shikhmurzaev, Y. D. (2012). The Dynamics of Liquid Drops and their Interaction with Solids of Varying Wettabilities. Submitted, páginas 1-27.

[48] Stenberg, R. (1995). On some techniques for approximating boundary conditions in the finite element method. Journal of Computational and Applied Mathematics, 63(1-3):139-148.

[49] Sven Groß, A. R. (2007). An extended pressure finite element space for twophase incompressible flows with surface tension. Journal of Computational Physics, 224(1):40-58. 